\title{
A metric approach to a class of doubly nonlinear evolution equations and applications
}

\author{
Riccarda Rossi, AleXander Mielke and GiUseppe SaVAré
}

\begin{abstract}
This paper deals with the analysis of a class of doubly nonlinear evolution equations in the framework of a general metric space. We propose for such equations a suitable metric formulation (which in fact extends the notion of Curve of Maximal Slope for gradient flows in metric spaces, see [5]), and prove the existence of solutions for the related Cauchy problem by means of an approximation scheme by time discretization. Then, we apply our results to obtain the existence of solutions to abstract doubly nonlinear equations in reflexive Banach spaces. The metric approach is also exploited to analyze a class of evolution equations in $L^{1}$ spaces.
\end{abstract}

Mathematics Subject Classification (2000): 35K55 (primary); 49Q20, 58E99 (secondary).

\section{Introduction}

Let $B$ be a separable Banach space, $T$ a positive number, and let us consider two proper functionals $\Psi: B \rightarrow(-\infty,+\infty]$ and $\mathcal{E}:[0, T] \times B \rightarrow(-\infty,+\infty]$, such that

$\Psi$ is convex and lower semicontinuous, $\quad \Psi(v) \geq \Psi(0)=0 \quad \forall v \in B$, $\mathcal{E}(t, \cdot): B \rightarrow(-\infty,+\infty]$ is lower semicontinuous for a.e. $t \in(0, T)$.

Hereafter, we shall denote by $\|\cdot\|$ the norm on $B$ and by $\|\cdot\|_{*}$ the norm on the dual space $B^{\prime}$. We consider the abstract doubly nonlinear evolution equation

$$
\partial \Psi\left(u^{\prime}(t)\right)+\partial \mathcal{E}(t, u(t)) \ni 0 \quad \text { in } B^{\prime} \quad \text { a.e. in }(0, T),
$$

where $\partial \Psi$ denotes the subdifferential in the sense of convex analysis of $\Psi$, while we provisionally denote by $\partial \mathcal{E}$ a suitable version of the subdifferential of $\mathcal{E}$ with respect to the second variable (in fact, we take $\partial \mathcal{E}$ to be the convex subdifferential

Received May 14, 2007; accepted in revised form December 4, 2007. 
of $\mathcal{E}$ with respect to the variable $u$ if the functional $u \mapsto \mathcal{E}(t, u)$ is convex). Clearly, in the quadratic case $\Psi(u):=\frac{1}{2}\|u\|_{B}^{2}$ for all $u \in B$, (DNE) reduces to a gradient flow equation.

This differential inclusion in fact arises in several applicative contexts, ranging, among others, from thermomechanics (where it may be understood as a generalized balance relation, see e.g. $[25,43])$, to the modeling of rate-independent evolutions. Without going into details, we point out that, within the realm of these applications, the functional $\Psi$ may be interpreted as a dissipation potential, while $\mathcal{E}$ is an energy functional. Indeed, there is nowadays a quite wide literature on the analysis of (DNE), which we briefly review, distinguishing the case in which the functional $\Psi$ has a superlinear growth at infinity from the linear-growth case.

The most general well-posedness results (earlier ones were obtained in $[6,13$, $48]$ ), for the Cauchy problem associated with (DNE) in the superlinear case for $\Psi$ date back to the papers $[15,16]$, along with applications to a broad class of PDE models, for phase transition phenomena, which can be recast in the general form (DNE). In the setting of a Hilbert space in [15], and of a reflexive Banach space in [16], the existence of solutions to (DNE) is proved via approximation by time discretization, and passage to the limit by compactness and monotonicity techniques. In $[15,16]$, the functional $\mathcal{E}$ takes the form $\mathcal{E}(t, u):=\Phi(u)-\langle\ell(t), u\rangle$ for all $(t, u) \in(0, T) \times B, \Phi$ being a convex functional, so that the convex subdifferential in (DNE) is given by $\partial \mathcal{E}=\partial \Phi-\ell$. In fact, this crucial convexity assumption allows to exploit maximal monotone operator techniques. We recall that, in the same setting, long-time behavior results for (DNE) have recently been obtained in $[46,47]$.

In the linear-growth case, equation (DNE) arises in connection with rate-independent problems. Indeed, in such situations the dissipation functional $\Psi$ is positively homogeneous of degree 1 , whence $\partial \Psi(\lambda v)=\partial \Psi(v)$ for all $\lambda \geq 0$ and $v \in B$. Therefore, a solution to (DNE) remains a solution if the time variable is rescaled, thus modeling phenomena insensitive to changes in the time scale. Rate-independent models indeed occur, for instance, in elastoplasticity [19, 20, 24, 29-32], in damage [39], in the quasistatic evolution of fractures [21,28], in shape memory alloys [38,40,41], and in several other contexts, see the survey [33] and the references therein. Existence, approximation, uniqueness, and regularity of solutions to the Cauchy problem for (DNE) in the rate-independent case have been proved in $[35,42]$, again in the setting of a reflexive Banach space and of a smooth, convex energy functional $\mathcal{E}$. However, the aforementioned applications to continuum mechanics problems lead to possibly non smooth and (highly) non convex energy functionals, as well as to ambient spaces which are neither reflexive, nor dual of separable spaces (for example, $L^{1}$ spaces for shape memory alloys), or even lack a linear structure (like in the applications to fractures). In fact, the non convexity of $\mathcal{E}$ may be a counterpart of the latter feature, as shown in Section 3.3 later on by the example of a non convex functional defined on a Banach manifold. These considerations have indeed motivated the development of an abstract, energetic formulation of rate-independent problems in [42], which can be in fact given in a purely topological framework, see [28]. We may mention that, in the same spirit, global variational principles for doubly nonlinear evolution equations (both 
in the superlinear and in the rate-independent case), have been recently proposed in $[34,49,51]$.

However, the analysis of the doubly nonlinear equation (DNE) in the case of a non convex, non smooth energy functional and of a more general ambient space still remains open. In the fully general case, one may indeed face the problem of giving meaning to the pointwise formulation (DNE) itself. For example, if the space $B$ does not enjoy the Radon-Nikodým property (like in the case of $L^{1}$ spaces), absolutely continuous curves with values in $B$ need not be a.e. differentiable with respect to the variable $t$, so that the pointwise time derivative appearing in (DNE) may not be defined. Furthermore, in the absence of a linear structure on the ambient space, the notion of (Gâteaux)-derivative/subdifferential of a functional does not make sense anymore. These drawbacks can be overcome by resorting to suitable surrogates of derivative notions, which have been introduced in the realm of Analysis in Metric Spaces. In particular, we refer to the notions proposed within the theory of Curves of Maximal Slope for gradient flows in metric spaces, which was initiated by E. De Giorgi in the seminal paper [18] and has been subsequently developed in $[3,4,27]$ and in the recent monograph [5].

The goal of this paper is to analyze (DNE) in the framework of a general metric space. Indeed, we shall provide a suitable purely metric formulation of (DNE), in fact adapting the notion of Curve of Maximal Slope to the doubly nonlinear case. Then, we shall prove an existence and approximation result for the related Cauchy problem in the case of a superlinear dissipation functional.

In the forthcoming papers [36,37], we shall instead develop the analysis of rateindependent problems in a metric framework. More precisely, we shall study rateindependent metric evolutions as the vanishing viscosity limit of doubly nonlinear metric evolutions driven by a superlinear dissipation. Let us point out that this asymptotic analysis has been recently addressed in the paper [22], for general rateindependent problems in a finite-dimensional framework, as well as in [20], in the more specific case of quasistatic evolutions in plasticity with softening.

\section{The metric formulation}

For simplicity, we introduce the metric formulation of equation (DNE) when the functional $\mathcal{E}$ is independent of the $t$ variable, postponing the general discussion to Section 2.4. In order to give some insight into the metric approach, we first develop some heuristic calculations in a smooth case and with an ambient Banach space.

Hence, we suppose that $\Psi \in C^{1}(B)$, that its Fenchel-Moreau conjugate $\Psi^{*}$ is in $C^{1}\left(B^{\prime}\right)$ too, and that $\mathcal{E} \in \mathrm{C}^{1}(B)$ (nevertheless, we do not require $\mathcal{E}$ to be convex). Under these smoothness assumptions,

$$
\begin{aligned}
& \partial \Psi(u)=\{\mathrm{D} \Psi(u)\}, \quad \partial \mathcal{E}(u)=\{\mathrm{D} \mathcal{E}(u)\} \quad \text { for all } u \in B, \\
& \partial \Psi^{*}(v)=\left\{\mathrm{D} \Psi^{*}(v)\right\} \quad \text { for all } v \in B^{\prime} .
\end{aligned}
$$

Thus, the doubly nonlinear equation (DNE) turns out to be

$$
\mathrm{D} \Psi\left(u^{\prime}(t)\right)+\mathrm{D} \mathcal{E}(u(t))=0 \quad \text { for a.e. } t \in(0, T) .
$$


By a convex analysis argument, (1.1) is equivalent to

$$
\Psi\left(u^{\prime}(t)\right)+\Psi^{*}(-\mathrm{D} \mathcal{E}(u(t))) \leq\left\langle-\mathrm{D} \mathcal{E}(u(t)), u^{\prime}(t)\right\rangle \quad \text { for a.e. } t \in(0, T),
$$

(where $\langle\cdot, \cdot\rangle$ denotes the duality pairing between $B^{\prime}$ and $B$ ). Let us point out that (1.2) in fact holds as an equality, as the converse inequality is true thanks to the definition of $\Psi^{*}$; however, in view of the metric formulation we are going to introduce later on, we prefer to state (1.2) in this form. Now, taking into account the chain rule formula

$$
\left\langle\mathrm{DE}(u(t)), u^{\prime}(t)\right\rangle=\frac{d}{d t} \mathcal{E}(u(t)) \quad \text { for a.e. } t \in(0, T),
$$

we can equivalently rephrase (1.1) as

$$
\frac{d}{d t} \mathcal{E}(u(t)) \leq-\Psi\left(u^{\prime}(t)\right)-\Psi^{*}(-\mathrm{D} \mathcal{E}(u(t))) \quad \text { for a.e. } t \in(0, T),
$$

which again in fact holds as an equality. To fix ideas, let us choose

$$
\begin{aligned}
\Psi(u) & :=\frac{1}{p}\|u\|^{p} \forall u \in B, \quad \text { so that } \\
\Psi^{*}(v) & :=\frac{1}{q}\|v\|_{*}^{q} \forall v \in B^{\prime}, \quad 1<p<\infty, \frac{1}{p}+\frac{1}{q}=1 .
\end{aligned}
$$

Then, (1.4) becomes

$$
\frac{d}{d t} \mathcal{E}(u(t)) \leq-\frac{1}{p}\left\|u^{\prime}(t)\right\|^{p}-\frac{1}{q}\|-\mathrm{D} \mathcal{E}(u(t))\|_{*}^{q} \quad \text { for a.e. } t \in(0, T) .
$$

Let us point out that the above formulation highlights the role of the norms of the derivatives $u^{\prime}(t)$ and $-\mathrm{DE}(t, u(t))$, rather than of the derivatives themselves. That is why, (1.4) appears to be a suitable formulation for going over to a purely metric framework, where one may (only) dispose of notions surrogating the norm of the pointwise derivative of a curve, and the norm of the Gâteaux derivative of a functional.

We now briefly recall such notions (referring to [3,4], [5, Chapter 1], and to the next sections for further details), in the context of a (separable) metric space $(X, d)$. In this framework, it is possible to define the notion of absolute continuity of a curve with values in $X$, and to prove that, if $u:(0, T) \rightarrow X$ is absolutely continuous, the limit

$$
\left|u^{\prime}\right|(t):=\lim _{h \rightarrow 0} \frac{d(u(t), u(t+h))}{h} \text { exists for a.e. } t \in(0, T),
$$

defining the metric derivative of the curve $u$. It can be readily checked that, if $X$ is a Banach space $B$ and if the absolutely continuous curve $u:(0, T) \rightarrow B$ is a.e. differentiable on $(0, T)$, then

$$
\left|u^{\prime}\right|(t)=\left\|u^{\prime}(t)\right\| \quad \text { for a.e. } t \in(0, T) .
$$


Nonetheless, as we are going to see in Section 7, the notion of metric derivative is significant even in spaces like $L^{1}$, in which the link (1.6) between the metric and the pointwise derivatives is no longer available for general absolutely continuous curves.

In the same way, given a functional $\mathcal{E}: X \rightarrow(-\infty,+\infty]$ and a point $u \in$ $\operatorname{dom}(\mathcal{E})$, following [18] we define the (local) slope of $\mathcal{E}$ at $u$ as

$$
|\partial \mathcal{E}|(u):=\limsup _{v \rightarrow u} \frac{(\mathcal{E}(u)-\mathcal{E}(v))^{+}}{d(u, v)} .
$$

Again, it can be shown that, in the Banach space case,

if $\mathcal{E}: B \rightarrow(-\infty,+\infty]$ is (Fréchet) differentiable at $u \in \operatorname{dom}(\mathcal{E})$,

$$
\text { then }|\partial \mathcal{E}|(u)=\|\mathrm{D} \mathcal{E}(u)\|_{*} \text {. }
$$

More in general, if $\mathcal{E}$ is convex, then $|\partial \mathcal{E}|$ is related to the (convex) subdifferential $\partial \mathcal{E}$ of $\mathcal{E}$ by

$$
|\partial \mathcal{E}|(u)=\min \left\{\|\xi\|_{*}: \xi \in \partial \mathcal{E}(u)\right\} \quad \forall u \in \operatorname{dom}(\partial \mathcal{E}) .
$$

The chain rule formula (1.3) translates in the metric setting as

$$
\begin{gathered}
\frac{d}{d t} \mathcal{E}(u(t)) \geq-\left|u^{\prime}\right|(t) \cdot|\partial \mathcal{E}|(u(t)) \quad \text { for a.e. } t \in(0, T) \\
\text { for any absolutely continuous curve } u:(0, T) \rightarrow X .
\end{gathered}
$$

We remark that, in the case of a smooth functional $\mathcal{E}: B \rightarrow(-\infty,+\infty]$, the above chain rule inequality results from (1.3), (1.8), and the Cauchy-Schwarz inequality; we have the same interpretation in the case of a convex functional as well, due to (1.9) and the well-known chain rule for the subdifferential in the sense of convex analysis. We refer to Section 2.4 for a detailed discussion of the chain rule (1.10) in the case of a time-dependent functional.

We are now in the position of stating the metric analog of (1.4), of course replacing the derivatives of the curve and of the functional with the metric derivative (1.5) and the local slope (1.7). Since we are now dealing with scalar notions, the role of the dissipation $\Psi$ shall be played by a function

$$
\begin{aligned}
& \psi:[0,+\infty) \rightarrow[0,+\infty), \quad \text { convex and lower semicontinuous, } \\
& \text { with } \psi(0)=0 \text { and } \lim _{x \rightarrow+\infty} \frac{\psi(x)}{x}=+\infty .
\end{aligned}
$$

Hence, supposing that the functional $\mathcal{E}: X \rightarrow(-\infty,+\infty]$ complies with the chain rule (1.10), we say that an absolutely continuous curve

$u:(0, T) \rightarrow X$ satisfies the metric formulation of (DNE) if the map $t \in(0, T) \mapsto \mathcal{E}(u(t))$ is absolutely continuous and $\frac{d}{d t} \mathcal{E}(u(t)) \leq-\psi\left(\left|u^{\prime}\right|(t)\right)-\psi^{*}(|\partial \mathcal{E}|(u(t))) \quad$ for a.e. $t \in(0, T)$. 
It can be easily checked that, when $\psi(x)=\frac{1}{2} x^{2}$ for all $x \in[0,+\infty)$, the above formulation coincides with the metric formulation of gradient flows, see $[4,5,18]$.

The main result of this paper (Theorem 3.5 later on) states the existence of absolutely continuous curves complying with the above metric formulation, supplemented by an initial condition. The proof is performed by passing to the limit in an approximation scheme based on time discretization (see Section 3.1). The variational scheme yielding the approximate solutions is indeed the metric analog of the implicit Euler scheme used for doubly nonlinear evolution equations in Banach spaces (see $[15,16,42]$ ). As a matter of fact, such a scheme has been proposed in $[4,17]$ as a possible way to approximate gradient flows in metric spaces, in the framework of the theory of Minimizing Movements. Exploiting some technical tools of this theory, we have been able to show that the approximate solutions converge to a curve solving the Cauchy problem for (1.12). Without going into many details, let us point out that the whole procedure works out under some lower semicontinuity and coercivity assumptions on $\mathcal{E}$ (which substantially enable to carry out the approximation scheme and to obtain compactness of the approximate solutions), joint with the lower semicontinuity of the map $u \mapsto|\partial \mathcal{E}|(u)$ and the chain rule (1.10).

\section{Applications: the reflexive case}

The main applications of our metric approach are to (a class of) doubly nonlinear evolution equations of the form

$$
\partial \Psi\left(u(t), u^{\prime}(t)\right)+\partial \mathcal{E}(t, u(t)) \ni 0 \quad \text { in } B^{\prime} \quad \text { for a.e. } t \in(0, T),
$$

in the setting of

$$
\text { a separable reflexive Banach space } B \text {, }
$$

the functional $\Psi$ having a superlinear growth with respect to the second variable. Existence results for a class of equations of the type (1.13) (which are often called quasivariational due to the dependence of the dissipation functional on the state variable $u$ ), have been recently obtained in the papers $[7,8]$ for a superlinear dissipation, while the quasivariational rate-independent case has been analyzed in [35]. In fact, in this paper we are able to deal with dissipation functionals in (1.13) of the form

$$
\Psi(u, v):=\psi\left(\|v\|_{u}\right) \quad \forall u, v \in B,
$$

where $\psi$ is as in (1.11) and

$\left\{\|\cdot\|_{u}\right\}_{u \in B}$ is a family of norms on $B$, inducing the Finsler distance

$d(v, w):=\inf \left\{\int_{0}^{1}\left\|u^{\prime}(t)\right\|_{u(t)} \mathrm{d} t: u:[0,1] \rightarrow B, u(0)=v, u(1)=w\right\} \forall v, w \in B$. 
In the setting of the metric space $(B, d)$, it is possible to prove that relation (1.6) between the metric and the pointwise derivative of an absolutely continuous curve still holds in a suitable form (see Section 6). Likewise, relations (1.8)-(1.9) between the slope and the (sub-)differential of $\mathcal{E}$ carry over to this Finsler setting if $\mathcal{E}$ is, for instance, a $\lambda$-convex or a $\mathrm{C}^{1}$ perturbation of a convex functional. As a result, every absolutely continuous curve fulfilling the metric formulation (1.12) is in fact a solution of the pointwise differential inclusion (1.13). Thus, our main metric result, Theorem 3.5, yields the existence of absolutely continuous curves solving the Cauchy problem related to (1.13), $c f$. Theorem 8.3 later on. A typical parabolic evolution equation which can be rephrased in the abstract form (1.13), with $B=$ $L^{p}(\Omega)$, is the following generalized Allen-Cahn equation

$$
\alpha\left(u, u_{t}\right)\left|u_{t}\right|^{p-2} u_{t}-\Delta u+u^{3}-u=h \quad \text { a.e. in } \Omega \times(0, T),
$$

$u_{t}$ denoting the partial time derivative of $u$. Here, $p \geq 1, \Omega$ is a bounded domain in $\mathbb{R}^{d}, d \geq 1, \alpha: \mathbb{R}^{2} \rightarrow(0,+\infty)$ a continuous function, bounded from below and from above, $\Delta$ is the Laplace operator, and $h: \Omega \times(0, T) \rightarrow \mathbb{R}$ some source term. In fact, (1.15) is the prototype of the parabolic doubly nonlinear equations we shall address in Section 8.2. More precisely, we shall deduce from Theorem 8.3 the existence of solutions to a suitable initial-boundary value problem for (a generalization of) (1.15).

\section{Applications: the $L^{1}$ case}

We shall also apply our metric approach to the analysis of (doubly nonlinear) metric evolutions in the metric space $L^{1}(\Omega)$, with the distance induced by the $L^{1}(\Omega)$-norm. As already mentioned, absolutely continuous curves on a time interval $(0, T)$ with values in $L^{1}(\Omega)$ are not, in general, a.e. differentiable on $(0, T)$, so that the metric formulation (1.12) does not lead to a pointwise formulation any more. Hence, in Section 7 we shall focus on purely metric evolutions only, in the case of the dissipation functional

$$
\psi(x):=\frac{1}{2} x^{2} \quad \forall x \geq 0
$$

In fact, in Section 7.2 we shall analyze the evolution driven in $L^{1}(\Omega)$, by the quadratic $\psi$ (1.16) and by an energy functional of Ginzburg-Landau type, and prove an existence result (Theorem 7.5). However, in Section 7.1 we shall preliminarily compare the metric and the pointwise formulations on some simpler examples. For instance, we shall consider, in the case $\Omega=(0,1)$, the quadratic energy functional $\mathcal{E}: L^{1}(0,1) \rightarrow[0,+\infty]$

$$
\mathcal{E}(u):=\left\{\begin{array}{ll}
\frac{1}{2} \int_{0}^{1} u^{2}(x) \mathrm{d} x & \text { if } u \in L^{2}(0,1), \\
+\infty & \text { otherwise }
\end{array} \quad \forall u \in L^{1}(0,1)\right.
$$


It can be checked that the local slope of $\mathcal{E}$ in the metric space $L^{1}(0,1)$ is

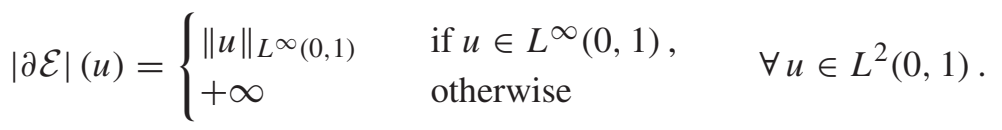

Hence, an absolutely continuous curve $u:(0, T) \rightarrow L^{1}(0,1)$ fulfills the metric formulation (1.12) in $L^{1}(0,1)$, with the choices (1.16)-(1.17), if

$$
\begin{aligned}
& \text { the map } t \in(0, T) \mapsto \frac{1}{2} \int_{0}^{1} u^{2}(x, t) \mathrm{d} x \text { is absolutely continuous and } \\
& \frac{d}{d t}\left(\int_{0}^{1} u^{2}(x, t) \mathrm{d} x\right) \leq-\left|u^{\prime}\right|(t)^{2}-\|u(t)\|_{L^{\infty}(0,1)}^{2} \quad \text { for a.e. } t \in(0, T) .
\end{aligned}
$$

On the other hand, the evolution equation corresponding to (1.18) is (DNE), driven by the dissipation potential

$$
\Psi(u):=\frac{1}{2}\|u\|_{1}^{2} \quad \forall u \in L^{1}(\Omega)
$$

and by the energy functional $\mathcal{E}(1.17)$, namely

$$
\left\|u_{t}(t)\right\|_{1} \operatorname{Sign}\left(u_{t}(x, t)\right)+u(x, t) \ni 0 \quad \text { for a.e. }(x, t) \in(0,1) \times(0, T)
$$

where we denote by Sign the multivalued operator

$$
\operatorname{Sign}(r):= \begin{cases}1 & \text { if } r>0, \\ {[-1,1]} & \text { if } r=0, . \\ -1 & \text { if } r<0\end{cases}
$$

As a matter of fact, in Section 7.1, we shall calculate an explicit solution of (the Cauchy problem for) (1.20) and show that it also fulfills the metric formulation (1.18).

\section{Plan of the paper}

In Section 2 we fix the metric setup in which we are going to develop our theory, and accordingly give the preliminary definitions of metric derivative, slope, and chain rule condition. In fact, we extend these notions to the framework of a nonsymmetric distance $\Delta$ on the space $X$, and we also allow $\Delta$ to take value $+\infty$. Further, besides the topology induced by $\Delta$, we are also going to deal with another topology $\sigma$ on $X$, possibly weaker, which mimics the role of the weak topology in the Banach space case. Section 3 is devoted to the construction of the approximation scheme for (the Cauchy problem related to) (1.12), and to the statement of our existence and approximation Theorem 3.5. Subsequently, we illustrate such 
result on the simple example of a time-independent functional defined on an infinite dimensional Banach manifold. The proof of Theorem 3.5 is carried out in several steps in Section 4. Starting from Section 5, we develop the main applications of our results to the Banach space setting. Indeed, Sections 5 and 6 are devoted to preliminaries, the former in the setting of a separable Banach space, and the latter of a separable reflexive Banach space, endowed with a Finsler (asymmetric) distance induced by a family of sublinear functionals (in fact, a generalization of the setup (1.14)). Throughout these sections, we investigate the link between slopes and subdifferentials, and prove (a version of) formula (1.9) for a broad class of functionals, encompassing $\lambda$-convex functionals and $\mathrm{C}^{1}$-perturbations of convex functionals. We show that these functionals also comply with the chain rule (1.10) in the general Banach space case. Moreover, we provide some technical results enabling to switch from the metric formulation (1.12) back to the pointwise formulation (1.13) in the Finsler, reflexive case. Building on the material developed in Section 5, in Section 7 we investigate metric evolutions in $L^{1}$ spaces and also provide some examples with explicit computations of the metric solution. Finally, on the basis of Section 6, in Section 8 we develop the aforementioned applications first in the setting of a general reflexive Banach space, with a Finsler asymmetric distance, secondly in the space $L^{p}(\Omega), 1<p<\infty$.

ACKNOWLEDGEMENTS. The research was partially supported by the European Union under HPRN-CT-2002-00284 Smart Systems: New Materials, Adaptive Systems and their Nonlinearities, by the Deutsche Forschungsgemeinschaft via SFB 404 Multifield Problems in Solid and Fluid Mechanics (subproject C7), and by grants of the M.I.U.R. (PRIN2004 Modellizzazione Matematica ed Analisi dei Problemi a Frontiera Libera). The authors are grateful for stimulating discussion with Messoud Efendiev; R. R. and G. S. acknowledge the kind hospitality of the Institut für Analysis, Dynamik und Modellierung, Universität Stuttgart, where this research was initiated. 


\section{Part I: the metric theory}

\section{The metric setup}

\subsection{Asymmetric distances and metric derivatives}

\section{General assumptions}

In a

$$
\text { Hausdorff topological space }(X, \sigma) \text {, }
$$

we are given a reference point $x_{o} \in X$ and

a possibly non symmetric (asymmetric) distance $\Delta: X \times X \rightarrow[0,+\infty]$, fulfilling

$$
\begin{array}{cl}
\Delta(u, v)=0 \quad \Leftrightarrow \quad u=v & \forall u, v \in X, \\
\Delta(u, v) \leq \Delta(u, w)+\Delta(w, v) & \forall u, v, w \in X,
\end{array}
$$

We set

$$
\delta(u, v):=\min [\Delta(u, v), \Delta(v, u)], X_{u}:=\{v \in X: \Delta(u, v)<+\infty\}, X_{0}:=X_{x_{o}} .
$$

Observe that we could always assume that $\Delta$ is finite, by restricting our discussion to the space $X_{0}$; nevertheless, sometimes it could be useful to allow a more flexible choice of the reference point $x_{0}$.

Remark 2.1. A typical non-symmetric distance $\Delta$ allowed to take the value $+\infty$ is defined on the space $X=L^{1}(\Omega), \Omega$ being a measurable subset of $R^{d}$, by

$$
\Delta(u, v)= \begin{cases}\|u-v\|_{L^{1}} & \text { if } u \geq v \text { a.e. in } \Omega, \quad \forall u, v \in L^{1}(\Omega) . \\ +\infty & \text { else }\end{cases}
$$

Indeed, this example is relevant within applications to damage problems, see [39].

\section{Metric $\Delta$-derivatives}

It is easy to extend the notion of metric derivative (see [3]) of an absolutely continuous curve in $X$ to a possibly nonsymmetric setting.

First, for $1 \leq p \leq+\infty$ we define

$$
\begin{aligned}
& \operatorname{AC}^{p}(a, b ; X) \\
& :=\left\{v:(a, b) \rightarrow X: \exists m \in L^{p}(a, b) \text { s.t. } \Delta(v(s), v(t)) \leq \int_{s}^{t} m(r) \mathrm{d} r\right\},
\end{aligned}
$$

denoting by $\operatorname{AC}(a, b ; X)$ the space $\operatorname{AC}^{1}(a, b ; X)$. Note that, if one disposes of a distance $d$ on the ambient space $X$ fulfilling

$$
\exists \kappa_{1}, \kappa_{2}>0: \quad \kappa_{1} d(u, v) \leq \delta(u, v) \leq \kappa_{2} d(u, v) \quad \forall u, v, \in X .
$$


then $\operatorname{AC}^{p}(a, b ; X)$ is included in the usual space of absolutely continuous curves in the metric space $(X, d)$ (see [3]).

The following result is the natural extension of [5, Theorem 1.1.2].

Proposition 2.2. For any $v \in \operatorname{AC}^{p}(a, b ; X), 1 \leq p \leq+\infty$, the limits

$$
\left|v^{\prime}\right|(t):=\lim _{h \downarrow 0} \frac{\Delta(v(t), v(t+h))}{h}=\lim _{h \downarrow 0} \frac{\Delta(v(t-h), v(t))}{h}
$$

exist and are equal for a.e. $t \in(a, b)$; the function $\left|v^{\prime}\right|$ is in $L^{p}(a, b)$ and fulfills

$$
\Delta(v(s), v(t)) \leq \int_{s}^{t}\left|v^{\prime}\right|(r) \mathrm{d} r \quad \forall a<s \leq t<b .
$$

Furthermore,

$$
\left|v^{\prime}\right|(t) \leq m(t) \text { for a.e. } t \in(a, b)
$$

for any function $m \in L^{p}(a, b)$ fulfilling

$$
\Delta(v(s), v(t)) \leq \int_{s}^{t} m(r) \mathrm{d} r \quad \forall a<s \leq t<b .
$$

Proof. Let us fix $v \in \operatorname{AC}^{p}(a, b ; X)$ and let $m \in L^{p}(a, b)$ fulfill (2.8). Let us introduce for any $s \in(a, b)$ the function $l_{s}:(a, b) \rightarrow[0,+\infty)$ by

$$
l_{s}(t):=\Delta(v(s), v(t)) \quad \forall t \in(a, b) .
$$

By the definition (2.4) of $\mathrm{AC}^{p}(a, b ; X)$, we get the following inequality

$$
\left(l_{s}\left(t_{2}\right)-l_{s}\left(t_{1}\right)\right)^{+} \leq \Delta\left(v\left(t_{1}\right), v\left(t_{2}\right)\right) \leq \int_{t_{1}}^{t_{2}} m(r) \mathrm{d} r \quad \forall a<t_{1} \leq t_{2}<b,
$$

whence we deduce that the map $t \mapsto g_{s}(t):=l_{s}(t)-\int_{a}^{t} m(r) \mathrm{d} r$ is non increasing on $(a, b)$, in particular a.e. differentiable. Moreover, from (2.9) we get

$$
\left(l_{s}^{\prime}(t)\right)^{+} \leq \ell(t):=\liminf _{h \downarrow 0} \frac{\Delta(v(t), v(t+h))}{h} \quad \text { for a.e. } t \in(a, b) .
$$

Note that $\ell$ is a measurable positive function on $(a, b)$, fulfilling

$$
0 \leq \ell(t) \leq \liminf _{h \downarrow 0} \frac{1}{h} \int_{t}^{t+h} m(r) \mathrm{d} r=m(t) \quad \text { for a.e. } t \in(a, b) .
$$

Thus, $\ell \in L^{p}(a, b)$; moreover, since $l_{s}(t)=\int_{a}^{t} m(r) d r+g_{s}(t)$ and $g$ is non increasing, the singular part of the distributional derivative of $l_{s}$ is a non positive measure and therefore (2.10) yields

$$
\Delta(v(s), v(t))=l_{s}(t) \leq \int_{s}^{t}\left(l_{s}^{\prime}(r)\right)^{+} \mathrm{d} r \leq \int_{s}^{t} \ell(r) \mathrm{d} r \quad \forall a<s \leq t<b .
$$


Further, let us consider the measurable function $\tilde{\ell}:(a, b) \rightarrow[0,+\infty)$ defined by

$$
\tilde{\ell}(t):=\limsup _{h \downarrow 0} \frac{\Delta(v(t), v(t+h))}{h} \quad \forall t \in(a, b) .
$$

Arguing as in (2.10), we deduce from (2.8) that

$$
\tilde{\ell}(t) \leq \tilde{m}(t) \text { for a.e. } t \in(a, b)
$$

for any $\tilde{m} \in L^{p}(a, b)$ for which inequality (2.8) holds. Thus, due to (2.12) we find

$$
\tilde{\ell}(t) \leq \ell(t), \quad \text { hence } \quad \exists\left|v^{\prime}\right|(t):=\lim _{h \downarrow 0} \frac{\Delta(v(t), v(t+h))}{h} \quad \text { for a.e. } t \in(a, b),
$$

and $\left|v^{\prime}\right|(t) \in L^{p}(a, b)$ by (2.11). Moreover, (2.12) yields (2.6). We have thus proved the first part of the statement. The second one follows from (2.13).

Finally, the existence of the second limit of (2.5) follows from the same argument, applied to the reversed curve $\hat{v}(t):=v(a+b-t)$ and to the reversed distance $\hat{\Delta}(u, v):=\Delta(v, u)$. In particular

$$
\begin{aligned}
\Delta(v(s), v(t)) & =\hat{\Delta}(\hat{v}(a+b-t), \hat{v}(a+b-s)) \leq \int_{a+b-t}^{a+b-s}\left|\hat{v}^{\prime}\right|(r) \mathrm{d} r \\
& =\int_{s}^{t}\left|\hat{v}^{\prime}\right|(a+b-r) \mathrm{d} r .
\end{aligned}
$$

By the minimality property (2.7) (applied to $v$ and $\hat{v}$ ) we get

$$
\left|v^{\prime}\right|(t)=\left|\hat{v}^{\prime}\right|(a+b-t) \quad \text { for a.e. } t \in(a, b),
$$

which yields the identity between the two limits in (2.5).

\section{2. $\Delta$-slopes}

In the setup specified by (2.1)-(2.2), let $\mathcal{E}: X \rightarrow(-\infty,+\infty]$ be a functional with proper domain $E=\operatorname{dom}(\mathcal{E})=\{u \in X: \mathcal{E}(u)<+\infty\}$. Hereafter, we shall suppose that

$$
\mathcal{E} \text { is } \sigma \text {-sequentially lower semicontinuous. }
$$

We now introduce the notion of local and relaxed slope in the framework of the asymmetric distance $\Delta$ : the following definition mimics in an obvious way the usual definitions of slope given in the setting of a symmetric distance, for which we refer to $[3,5]$.

Definition 2.3. The $\Delta$-local slope of the functional $\mathcal{E}$ at a point $u \in \operatorname{dom}(\mathcal{E})$ is

$$
|\partial \mathcal{E}|(u):=\limsup _{\Delta(u, v) \rightarrow 0} \frac{(\mathcal{E}(u)-\mathcal{E}(v))^{+}}{\Delta(u, v)} .
$$


The $\Delta$-relaxed slope $\left|\partial^{-} \mathcal{E}\right|$ of $\mathcal{E}$ at a point $u \in \operatorname{dom}(\mathcal{E}) \cap X_{0}$ is defined by

$$
\left|\partial^{-} \mathcal{E}\right|(u):=\inf \left\{\liminf _{n \uparrow \infty}|\partial \mathcal{E}|\left(u_{n}\right): \quad u_{n} \stackrel{\sigma}{\rightarrow} u, \quad \sup \left\{\Delta\left(x_{o}, u_{n}\right), \mathcal{E}\left(u_{n}\right)\right\}<+\infty\right\} .
$$

Note that if $\mathrm{D} \cap X_{u}=\{u\}$ then $|\partial \mathcal{E}|(u)=0 ;\left|\partial^{-} \mathcal{E}\right|$ is (a version of) the (sequential) lower semicontinuous envelope of $|\partial \mathcal{E}|$ with respect to to the topology $\sigma$, along sequences in $X_{0}$ of bounded energy and bounded distance with respect to $x_{o}$.

\subsection{Time dependent families of energy functionals}

In this paper we deal with families of time-dependent functionals $\mathcal{E}_{t}: X \rightarrow(-\infty,+\infty]$, $t \in[0, T]$. In order to avoid further technical difficulties, we will only consider a quite "regular" dependence with respect to time: we thus assume that the proper domain of $\mathcal{E}_{t}$ is fixed, i.e.

$$
\mathrm{D}:=\operatorname{dom}\left(\mathcal{E}_{t}\right) \quad \forall t \in[0, T], \quad \text { and we set } \quad D_{0}:=\mathrm{D} \cap X_{0} .
$$

We also suppose that the functionals are uniformly bounded from below, letting

$$
-C_{0}:=\inf _{t \in[0, T], v \in \mathrm{D}} \mathcal{E}_{t}(v)>-\infty,
$$

and that

$\forall v \in \mathrm{D}$ the function $t \mapsto \mathcal{E}_{t}(v)$ is differentiable in $[0, T]$, with derivative $\partial_{t} \mathcal{E}_{t}(v)$

which satisfies

$$
\left|\partial_{t} \mathcal{E}_{t}(v)\right| \leq C_{1}\left(\mathcal{E}_{t}(v)+\Delta\left(x_{o}, v\right)+2 C_{0}\right) \quad \forall t \in[0, T], v \in D_{0}
$$

for a suitable constant $C_{1} \geq 0$.

Remark 2.4. Let us point out that (2.19d) (which has been proposed in [33, §3]), and the Gronwall Lemma yield the following estimate

$$
\begin{gathered}
\mathcal{E}_{t}(v) \leq\left(\mathcal{E}_{s}(v)+2 C_{0} C_{1}|t-s|+C_{1} \Delta\left(x_{o}, v\right)|t-s|\right) \exp \left(C_{1}|t-s|\right) \\
\forall t, s \in[0, T], v \in D_{0} .
\end{gathered}
$$

We will often impose some lower semicontinuity-compactness conditions on sequences with equibounded energy $\mathcal{E}_{t}$; thanks to the previous remark, the particular choice of the time $t$ is not relevant, so that we can state our assumptions for a fixed arbitrary time. We thus introduce the auxiliary quantity

$$
\mathcal{F}(v):=2 C_{0}+\Delta\left(x_{o}, v\right)+\mathcal{E}_{0}(v)
$$


and note that we have, for a suitable constant $C>0$,

$$
\begin{aligned}
& \frac{1}{C} \sup _{t \in[0, T]}\left(2 C_{0}+\Delta\left(x_{o}, v\right)+\mathcal{E}_{t}(v)\right) \\
& \leq \mathcal{F}(v) \leq C \inf _{t \in[0, T]}\left(2 C_{0}+\Delta\left(x_{o}, v\right)+\mathcal{E}_{t}(v)\right) \quad \forall v \in D_{0} .
\end{aligned}
$$

Therefore, the $t$-energy $\mathcal{E}_{t}\left(u_{n}\right)$ of a $\Delta$-bounded sequence $\left\{u_{n}\right\} \subset D_{0}$ remains bounded if and only if $\sup _{n} \mathcal{F}\left(u_{n}\right)<+\infty$ : in this case $\sup _{n \in \mathbb{N}, s \in[0, T]} \mathcal{E}_{s}\left(u_{n}\right)<+\infty$.

\subsection{The purely metric formulation of the Cauchy Problem (DNE)}

\section{Chain rule and curves of maximal slope}

It is not difficult to check that if $v:[0, T] \rightarrow \mathrm{D}$ is a curve and $\bar{t} \in(0, T)$ is a point such that there exists the metric $\Delta$-derivative $\left|v^{\prime}\right|(\bar{t})$, the map $t \mapsto \mathcal{E}_{t}(v(t))$ is continuously differentiable at $\bar{t}$, and $\left|\partial \mathcal{E}_{\bar{t}}\right|(v(\bar{t}))<+\infty$, then

$$
\left.\frac{d}{d t} \mathcal{E}_{t}(v(t))\right|_{t=\bar{t}} \geq \partial_{t} \mathcal{E}_{\bar{t}}(v(\bar{t}))-\left|v^{\prime}\right|(\bar{t}) \cdot\left|\partial \mathcal{E}_{\bar{t}}\right|(v(\bar{t})) .
$$

In this paper we are interested in finding curves of maximal slope, i.e. attaining equality in (2.23):

$$
\left.\frac{d}{d t} \mathcal{E}_{t}(v(t))\right|_{t=\bar{t}}=\partial_{t} \mathcal{E}_{\bar{t}}(v(\bar{t}))-\left|v^{\prime}\right|(\bar{t}) \cdot\left|\partial \mathcal{E}_{\bar{t}}\right|(v(\bar{t})) .
$$

In a linear Euclidean framework, this would be equivalent to imposing that the velocity vector and the gradient of the functional $\mathcal{E}_{t}$ have opposite directions at each time. Of course, we should complement this condition with a relation between their moduli of the type

$$
\left|\partial \mathcal{E}_{\bar{t}}\right|(v(\bar{t}))=h\left(\left|v^{\prime}\right|(\bar{t})\right)
$$

$h:[0,+\infty) \rightarrow[0,+\infty)$ being a continuous, surjective, and increasing map. By introducing its convex primitive function and its Legendre-Fenchel-Moreau transform

$$
\psi(x):=\int_{0}^{x} h(r) \mathrm{d} r, \quad \psi^{*}(y)=\sup _{x \geq 0} x y-\psi(x),
$$

and recalling that for arbitrary couples of non negative real numbers $x, y \geq 0$

$$
x y \leq \psi(x)+\psi^{*}(y), \quad y=h(x)=\psi^{\prime}(x) \Leftrightarrow x y=\psi(x)+\psi^{*}(y),
$$

we thus end up with the differential characterization

$$
\frac{d}{d t} \mathcal{E}_{t}(v(t))=\partial_{t} \mathcal{E}_{t}(v(t))-\psi\left(\left|v^{\prime}\right|(t)\right)-\psi^{*}\left(\left|\partial \mathcal{E}_{t}\right|(v(t))\right), \quad t \in(0, T) .
$$


We may further consider a relaxed version of (2.27): first of all, we would like to replace the slope $\left|\partial \mathcal{E}_{t}\right|$ with its lower semicontinuous envelope $\left|\partial^{-} \mathcal{E}_{t}\right|$ (2.18), which enjoys better convergence properties. This is meaningful only if $\left|\partial^{-} \mathcal{E}_{t}\right|$ is strong enough to control the time derivative of the energies $\mathcal{E}_{t}$ along absolutely continuous curves. We fix this property in the following definition:

Definition 2.5 (Chain rule for the relaxed slope). Let $\mathcal{E}_{t}, t \in[0, T]$, be a family of functionals fulfilling $(2.19 a, b, c, d)$. We say that $\left|\partial^{-} \mathcal{E}_{t}\right|$ satisfies the chain rule condition if for any curve $v \in \operatorname{AC}\left(0, T ; X_{0}\right)$ with

$$
\int_{0}^{T}\left|v^{\prime}\right|(t) \cdot\left|\partial^{-} \mathcal{E}_{t}\right|(v(t)) \mathrm{d} t<+\infty, \quad \sup _{t \in(0, T)} \mathcal{E}_{t}(v(t))<+\infty,
$$

the map $t \mapsto \mathcal{E}_{t}(v(t))$ is absolutely continuous, and

$$
\frac{d}{d t} \mathcal{E}_{t}(v(t)) \geq \partial_{t} \mathcal{E}(t, v(t))-\left|v^{\prime}\right|(t) \cdot\left|\partial^{-} \mathcal{E}_{t}\right|(v(t)) \quad \text { for a.e. } t \in(0, T) .
$$

We may also drop the continuity assumption on $h$, by considering monotone surjective graphs instead of maps: in this case, we simply replace the relation $h=\psi^{\prime}$ with the subdifferential condition $h=\partial \psi$ and (2.25) by

$$
\left|\partial \mathcal{E}_{t}\right|(v(t)) \in h\left(\left|v^{\prime}\right|(t)\right) .
$$

We can therefore consider an arbitrary real function

$\psi:[0,+\infty) \rightarrow[0,+\infty], \quad$ convex and lower semicontinuous,

$$
\text { with } \psi(0)=0, \quad \text { superlinear growth } \quad \lim _{x \uparrow+\infty} \frac{\psi(x)}{x}=+\infty \text {, }
$$

$$
\text { and non empty } \operatorname{int}(\operatorname{dom}(\psi))=(0, a), \quad a \in(0,+\infty] \text {. }
$$

Collecting all the above remarks, we can now state our metric formulation of (the Cauchy Problem related to) (DNE).

Problem 2.6 (Metric formulation of (DNE)). Suppose that the chain rule condition stated in Definition 2.5 holds true. Given $u_{0} \in D_{0}$, find a curve $u \in \operatorname{AC}\left(0, T ; X_{0}\right)$ such that

$$
\begin{aligned}
& u(0)=u_{0} \text {, the map } t \mapsto \mathcal{E}_{t}(u(t)) \text { is absolutely continuous on }(0, T), \text { and } \\
& \frac{d}{d t} \mathcal{E}_{t}(u(t)) \leq \partial_{t} \mathcal{E}_{t}(u(t))-\psi\left(\left|u^{\prime}\right|(t)\right)-\psi^{*}\left(\left|\partial^{-} \mathcal{E}_{t}\right|(u(t))\right) \text { for a.e. } t \in(0, T)
\end{aligned}
$$

For instance, the choice $\psi(x):=x^{p} / p, x \in[0,+\infty), p \geq 1$, with conjugate $\psi^{*}(x)=x^{p^{\prime}} / p^{\prime}, 1 / p+1 / p^{\prime}=1$, of course fits in this framework. In this case, (2.32) reduces to

$$
\frac{d}{d t} \mathcal{E}_{t}(u(t)) \leq \partial_{t} \mathcal{E}_{t}(u(t))-\frac{\left|u^{\prime}\right|^{p}(t)}{p}-\frac{\left|\partial^{-} \mathcal{E}_{t}\right|^{p^{\prime}}(u(t))}{p^{\prime}} .
$$


Finally, let us point out for later convenience that, by an elementary convex analysis argument, if the chain rule of Definition 2.5 is satisfied, then any absolutely continuous curve fulfilling (2.32) indeed fulfills for a.e. $t \in(0, T)$

$$
\begin{aligned}
\frac{d}{d t} \mathcal{E}_{t}(u(t))-\partial_{t} \mathcal{E}_{t}(u(t)) & =-\left|u^{\prime}\right|(t)\left|\partial^{-} \mathcal{E}_{t}\right|(u(t)) \\
& =-\psi\left(\left|u^{\prime}\right|(t)\right)-\psi^{*}\left(\left|\partial^{-} \mathcal{E}_{t}\right|(u(t))\right),
\end{aligned}
$$

namely the inequality in (2.32) is equivalent to an equality.

Remark 2.7 (Link with the metric theory of gradient flows.). Let us point out that our metric approach to (DNE) is tightly linked to the general theory developed in [5] (see also the references therein) for gradient flow equations in metric spaces. More precisely, following the terminology of [5, Chapter 1], the chain rule property of Definition 2.5 is (with slight changes) equivalent to requiring $\left|\partial^{-} \mathcal{E}\right|$ to be an upper gradient, whereas the metric analog of our definition of solution is the notion of curve of maximal slope.

\subsection{Topological assumptions}

Let us collect here all the topological assumptions relating the asymmetric distance $\Delta$ and the functionals $\psi$ and $\mathcal{E}_{t}, t \in[0, T]$, to the topology $\sigma$ of $X$.

Sequential semicontinuity. If $\left\{u_{n}\right\},\left\{v_{n}\right\}, u, v \in X_{0}$ satisfy

$$
\sup _{n}\left(\mathcal{F}\left(u_{n}\right)+\mathcal{F}\left(v_{n}\right)\right)<+\infty \text { and }\left(u_{n}, v_{n}\right) \stackrel{\sigma}{\rightarrow}(u, v),
$$

then

$$
\begin{gathered}
\liminf _{n \uparrow \infty} \Delta\left(u_{n}, v_{n}\right) \geq \Delta(u, v), \quad \liminf _{n \uparrow+\infty} \mathcal{E}_{t}\left(u_{n}\right) \geq \mathcal{E}_{t}(u) \quad \forall t \in[0, T], \\
\limsup _{n \uparrow+\infty} \partial_{t} \mathcal{E}_{t}\left(u_{n}\right) \leq \partial_{t} \mathcal{E}_{t}(u) \quad \forall t \in[0, T] .
\end{gathered}
$$

Strengthened sequential semicontinuity. Further, in the case in which $\partial \psi$ is not single valued (this means that $\psi$ is not differentiable in the interior of its domain, or that (cf. (2.30)) $a<\infty, \psi(a)<+\infty$ and $\left.\psi_{-}^{\prime}(a)<+\infty\right)$, we also assume that for every sequence $t_{n} \in[0, T], u_{n} \in D_{0}$ such that

$$
\sup _{n}\left(\mathcal{F}\left(u_{n}\right)+\left|\partial \mathcal{E}_{t_{n}}\right|\left(u_{n}\right)\right)<+\infty, \quad \Delta\left(u, u_{n}\right) \rightarrow 0, \quad t_{n} \downarrow t,
$$

we have

$$
\limsup _{n \uparrow+\infty} \frac{\mathcal{E}_{t_{n}}\left(u_{n}\right)-\mathcal{E}_{t}\left(u_{n}\right)}{t_{n}-t} \leq \partial_{t} \mathcal{E}_{t}(u) .
$$

Note that (2.36) surely holds if the following slightly stronger version of (2.35) is satisfied

$$
\sup _{n} \mathcal{F}\left(u_{n}\right)<+\infty, \Delta\left(u, u_{n}\right) \rightarrow 0, t_{n} \downarrow t \Rightarrow \limsup _{n \uparrow+\infty} \partial_{t} \mathcal{E}_{t_{n}}\left(u_{n}\right) \leq \partial_{t} \mathcal{E}_{t}(u) .
$$


Sequential compactness. If a sequence $\left\{u_{n}\right\} \subset X_{0}$ satisfies $\sup _{n \in \mathbb{N}} \mathcal{F}\left(u_{n}\right)<+\infty$, then

$$
\exists u \in X \text { and a subsequence }\left\{u_{n_{k}}\right\} \sigma \text {-converging to } u \text {. }
$$

A few remarks on the above assumptions are in order.

Remark 2.8 (Topology comparison). Due to (2.34) and (2.38), we have

$$
\sup _{n} \mathcal{F}\left(u_{n}\right)<+\infty, \quad \delta\left(u_{n}, u\right) \rightarrow 0 \quad \Rightarrow \quad u_{n} \stackrel{\sigma}{\rightarrow} u .
$$

In fact, any $\sigma$-sequential limit point $v$ of $u_{n}$ (whose existence follows from the compactness assumption) satisfies, by (2.34), $\delta(u, v) \leq \liminf _{n \uparrow+\infty} \delta\left(u, u_{n}\right)=$ $\lim _{n \uparrow+\infty} \delta\left(u, u_{n}\right)=0$. Thus, $v$ coincides with $u$.

Remark 2.9 ( $\Delta$-completeness of the sublevels of $\mathcal{E})$. It is not difficult to check that the sublevels of $\mathcal{E}$ satisfies the following completeness property with respect to the asymmetric distance $\Delta$ : any sequence $u_{n} \in X_{0}$ satisfies

$$
\lim _{n \rightarrow \infty} \sup _{m>0} \Delta\left(u_{n}, u_{n+m}\right)=0, \sup _{n} \mathcal{E}\left(u_{n}\right)<+\infty \Rightarrow \exists ! u: u_{n} \stackrel{\sigma}{\rightarrow} u, \lim _{n \rightarrow \infty} \Delta\left(u_{n}, u\right)=0 .
$$

\section{The main result}

We shall construct a solution $u \in \operatorname{AC}(0, T ; X)$ to Problem 2.6 by passing to the limit in a suitable approximation scheme by time discretization.

In the sequel, we adopt the convention of denoting by the symbols $C$ and $C^{\prime}$ all the accessory positive constants occurring in the estimates.

\subsection{Approximation}

We fix a time step $\tau>0$, to which there corresponds a partition

$$
\mathscr{P}_{\tau}:=\left\{t_{0}=0<t_{1}<\ldots<t_{n}<\ldots<t_{N-1}<T \leq t_{N}\right\}, \quad t_{n}:=n \tau, \quad N \in \mathbb{N},
$$

of the interval $(0, T)$. We consider the following recursive minimization scheme Problem 3.1 (Variational approximation scheme). Given $U_{\tau}^{0}:=u_{0}$, find $U_{\tau}^{1}, \ldots$ $\ldots, U_{\tau}^{N} \in X$ fulfilling

$$
U_{\tau}^{n} \in J_{\tau}\left(t_{n}, U_{\tau}^{n-1}\right):=\underset{u \in X}{\operatorname{Argmin}}\left\{\tau \psi\left(\frac{\Delta\left(U_{\tau}^{n-1}, u\right)}{\tau}\right)+\mathcal{E}_{t_{n}}(u)\right\},
$$

for $n=1, \ldots, N$. 
Lemma 3.2. Under the lower semicontinuity-compactness assumptions (2.19a)(2.19b) and (2.34)-(2.38) on $\mathcal{E}$, and the growth-convexity conditions (2.30) on $\psi$, for all $\tau>0$ and $u_{0} \in \mathrm{D}$ Problem 3.1 admits at least one solution $\left\{U_{\tau}^{n}\right\}_{n=1}^{N}$. Further, if $u_{0} \in D_{0}$ then $U_{\tau}^{n} \in D_{0}$ for all $n=1, \ldots, N$.

The proof is a standard application of the well-known direct method in the Calculus of Variations.

\section{Approximate solutions}

Let $\bar{U}_{\tau}$ and $\underline{U}_{\tau}$ be, respectively, the left-continuous and right-continuous piecewise constant interpolants of the values $\left\{U_{\tau}^{n}\right\}_{n=1}^{N}$ fulfilling $\bar{U}_{\tau}\left(t_{n}\right)=\underline{U}_{\tau}\left(t_{n}\right)=U_{\tau}^{n}$ for all $n=1, \ldots, N$, i.e.,

$$
\bar{U}_{\tau}(t)=U_{\tau}^{n} \forall t \in\left(t_{n-1}, t_{n}\right], \quad \underline{U}_{\tau}(t)=U_{\tau}^{n-1} \forall t \in\left[t_{n-1}, t_{n}\right), \quad n=1, \ldots, N .
$$

Furthermore, let $\bar{t}_{\tau}, \underline{\mathrm{t}}_{\tau}:[0, T] \rightarrow[0, T]$ be defined by

$\overline{\mathrm{t}}_{\tau}(0)=\underline{\mathrm{t}}_{\tau}(0):=0, \overline{\mathrm{t}}_{\tau}(t):=t_{k}$ for $t \in\left(t_{k-1}, t_{k}\right], \underline{\mathrm{t}}_{\tau}(t):=t_{k-1}$ for $t \in\left[t_{k-1}, t_{k}\right)$.

Of course, for every $t \in[0, T]$ we have $\overline{\mathrm{t}}_{\tau}(t) \downarrow t$ and $\underline{\mathrm{t}}_{\tau}(t) \uparrow t$ as $\tau \downarrow 0$.

We introduce another family of interpolants, due to E. De Giorgi, between the discrete values $U_{\tau}^{n}$.

Definition 3.3 (De Giorgi variational interpolants). We denote by $\widetilde{U}_{\tau}$ any interpolant of the discrete values $\left\{U_{\tau}^{n}\right\}_{n=0}^{N}$ obtained by solving the problem

$$
\left\{\begin{array}{l}
\widetilde{U}_{\tau}(0)=u_{0}, \quad \text { and, for } t=t_{n-1}+r \in\left(t_{n-1}, t_{n}\right], \\
\widetilde{U}_{\tau}(t) \in J_{r}\left(t, U_{\tau}^{n-1}\right):=\operatorname{Argmin}_{u \in X}\left\{r \psi\left(\frac{\Delta\left(U_{\tau}^{n-1}, u\right)}{r}\right)+\mathcal{E}_{t}(u)\right\}
\end{array}\right.
$$

such that the map $t \mapsto \widetilde{U}_{\tau}(t)$ is Lebesgue measurable in $(0, T)$.

Remark 3.4 (Measurability of $\widetilde{U}_{\tau}$ ). Since the map $s \mapsto J_{s}\left(t_{n-1}, U_{\tau}^{n-1}\right)$ is $\sigma$ compactly valued and upper semicontinuous, the existence of a measurable selection $\widetilde{U}_{\tau}\left(t_{n-1}+r\right) \in J_{r}\left(t_{n-1}+r, U_{\tau}^{n-1}\right), r \in\left(t_{n-1}, t_{n}\right]$, is ensured by [14, Corollary III.3, Theorem III.6].

Note that when $t=t_{n}$, the minimization scheme in (3.5) coincides with the one in (3.2), so that we can always assume that

$$
\tilde{U}_{\tau}\left(t_{n}\right)=\underline{U}_{\tau}\left(t_{n}\right)=\bar{U}_{\tau}\left(t_{n}\right)=U_{\tau}^{n}, \quad \text { for every } n=1, \ldots, N .
$$




\subsection{Statement of the main result}

Theorem 3.5 (Main existence and approximation result). In the metric framework discussed in Sections 2.1 and 2.2, let us suppose that $\psi$ complies with (2.30) and that the family of functionals $\mathcal{E}_{t}, t \in[0, T]$, satisfies (2.19a)-(2.19d), the topological assumptions (2.34)-(2.38) of Section 2.5, and the chain rule condition of Definition 2.5.

Then, for any $u_{0} \in D_{0}$ and any sequence $\tau_{n} \downarrow 0$ as $n \uparrow \infty$, there exists a subsequence (still labeled $\left.\tau_{n}\right)$ and a curve $u \in \operatorname{AC}\left(0, T ; X_{0}\right)$ such that

$$
\bar{U}_{\tau_{n}}(t) \stackrel{\sigma}{\rightarrow} u(t), \quad \underline{U}_{\tau_{n}}(t) \stackrel{\sigma}{\rightarrow} u(t), \quad \tilde{U}_{\tau_{n}}(t) \stackrel{\sigma}{\rightarrow} u(t) \quad \forall t \in[0, T],
$$

where

$$
u \text { is a solution to Problem 2.6, thus satisfying also (2.33), }
$$

and the energy identity

$$
\begin{aligned}
\int_{s}^{t} \psi\left(\left|u^{\prime}\right|(r)\right) \mathrm{d} r & +\int_{s}^{t} \psi^{*}\left(\left|\partial^{-} \mathcal{E}_{r}\right|(u(r))\right) \mathrm{d} r+\mathcal{E}_{t}(u(t)) \\
& =\mathcal{E}_{s}(u(s))+\int_{s}^{t} \partial_{t} \mathcal{E}_{r}(u(r)) \mathrm{d} r
\end{aligned}
$$

for every $s, t \in[0, T]$. In addition, the following convergences hold as $n \uparrow \infty$

$$
\begin{array}{cc}
\int_{0}^{t} \psi\left(\frac{\Delta\left(\underline{U}_{\tau_{n}}(r), \bar{U}_{\tau_{n}}(r)\right)}{\tau_{n}}\right) \mathrm{d} r \longrightarrow \int_{0}^{t} \psi\left(\left|u^{\prime}\right|(r)\right) \mathrm{d} r & \forall t \in[0, T], \\
\int_{0}^{t} \psi^{*}\left(\left|\partial \mathcal{E}_{r}\right|\left(\widetilde{U}_{\tau_{n}}(r)\right)\right) \mathrm{d} r \longrightarrow \int_{0}^{t} \psi^{*}\left(\left|\partial^{-} \mathcal{E}_{r}\right|(u(r))\right) \mathrm{d} r & \forall t \in[0, T], \\
\mathcal{E}_{\overline{\mathrm{t}}_{\tau_{n}}(t)}\left(\bar{U}_{\tau_{n}}(t)\right) \rightarrow \mathcal{E}_{t}(u(t)), \quad \mathcal{E}_{t}\left(\widetilde{U}_{\tau_{n}}(t)\right) \rightarrow \mathcal{E}_{t}(u(t)) & \forall t \in[0, T],
\end{array}
$$

and for a.e. $t \in(0, T)$

$$
\left\{\begin{array}{ll}
\liminf _{n \uparrow \infty}\left|\partial \mathcal{E}_{t}\right|\left(\widetilde{U}_{\tau_{n}}(t)\right)=\left|\partial^{-} \mathcal{E}_{t}\right|(u(t)) & \text { if }\left|u^{\prime}\right|(t) \neq 0, \\
\liminf _{n \uparrow \infty} \psi^{*}\left(\left|\partial \mathcal{E}_{t}\right|\left(\widetilde{U}_{\tau_{n}}(t)\right)\right)=\psi^{*}\left(\left|\partial^{-} \mathcal{E}_{t}\right|(u(t))\right)=0 & \text { if }\left|u^{\prime}\right|(t)=0
\end{array} .\right.
$$

Finally, let

$$
\mathscr{I}:=\left\{t \in(0, T):\left|u^{\prime}\right|(t) \neq 0\right\} .
$$

Then,

i) if $\psi^{*}$ as well has superlinear growth, i.e. $\lim _{x \uparrow+\infty} \frac{\psi^{*}(x)}{x}=+\infty$, we have the further convergence

$$
\left|\partial \mathcal{E}_{t}\right|\left(\widetilde{U}_{\tau_{n}}\right) \rightarrow\left|\partial^{-} \mathcal{E}_{t}\right|(u) \quad \text { in } L^{1}(\mathscr{I}) \text { as } n \uparrow \infty ;
$$


ii) in the general case, there exists a non increasing sequence $\left\{O_{n}\right\}_{n}$ of Borel subsets of $(0, T)$ such that $\cap_{n} O_{n}=\emptyset$ and, denoting by $I_{n}$ the indicator function of the set $(0, T) \backslash O_{n}$, there holds

$$
I_{n} \cdot\left|\partial \mathcal{E}_{t}\right|\left(\widetilde{U}_{\tau_{n}}\right) \rightarrow\left|\partial^{-} \mathcal{E}_{t}\right|(u) \quad \text { in } L^{1}(\mathscr{I}) \text { as } n \uparrow \infty .
$$

\subsection{An example on an infinite Banach manifold}

We consider two Hilbert spaces $V$ and $H$, such that $V$ is densely and compactly embedded in $H$ (we identify $H \equiv H^{\prime} \subset V^{\prime}$ ), and we denote by $(\cdot, \cdot)$ and $\|\cdot\|$ the scalar product and the norm in $H$ and by $\|\cdot\|_{V}$ the norm in $V$. We define the metric space $(X, \Delta)$ via

$$
X=\{u \in H:\|u\|=1\}, \quad \Delta\left(u_{1}, u_{2}\right):=\left\|u_{1}-u_{2}\right\| \quad \forall u_{1}, u_{2} \in X,
$$

and take as $\sigma$ the topology induced by the distance $\Delta$. We consider a functional $\widehat{\mathcal{E}} \in C^{1}(V)$ fulfilling

$$
\widehat{\mathcal{E}} \text { is convex and } \exists \Lambda_{1}, \Lambda_{2}>0 \text { s.t. } \quad \widehat{\mathcal{E}}(v) \geq \Lambda_{1}\|v\|_{V}^{2}-\Lambda_{2} \quad \forall v \in V,
$$

and we define $\mathcal{E}: X \rightarrow(-\infty,+\infty]$ by

$$
\mathcal{E}(u):=\left\{\begin{array}{ll}
\widehat{\mathcal{E}}(u) & \text { for } u \in V \cap X, \\
+\infty & \text { otherwise }
\end{array} \quad \forall u \in X .\right.
$$

The following results shed light on the local slope of $\mathcal{E}$ and on its chain rule properties.

Lemma 3.6. Under the above assumptions, we have for all $u \in \operatorname{dom}(\mathcal{E})$

$$
\begin{aligned}
& |\partial \mathcal{E}|(u)<+\infty \Leftrightarrow \mathrm{D} \widehat{\mathcal{E}}(u) \in H, \quad \text { and in this case } \\
& \left|\partial^{-} \mathcal{E}\right|(u)=|\partial \mathcal{E}|(u)=\|\mathrm{D} \widehat{\mathcal{E}}(u)-(\mathrm{D} \widehat{\mathcal{E}}(u), u) u\| .
\end{aligned}
$$

Proof. We fix $u \in \operatorname{dom}(\mathcal{E})$ and note that for all $w \in V \backslash\{0\}$ such that $(w, u)=0$ there exists a curve $\gamma:[-\rho, \rho] \rightarrow X$ with $\gamma \in \mathrm{C}^{1}([-\rho, \rho] ; V)$ and $\gamma^{\prime}(0)=w$. Then,

$$
\frac{\mathcal{E}(u)-\mathcal{E}(\gamma(r))}{\Delta(u, \gamma(r))} \rightarrow \frac{\mathrm{D} \widehat{\mathcal{E}}(u)[w]}{\|w\|} \quad \text { as } r \rightarrow 0 .
$$

Being $w$ arbitrary, we infer that

$$
\begin{aligned}
|\partial \mathcal{E}|(u) \geq \sup _{w \in V \backslash\{0\},(w, u)=0} \frac{\mathrm{D} \widehat{\mathcal{E}}(u)[w]}{\|w\|} & \geq \sup _{v \in V \backslash\{0\}} \frac{\mathrm{D} \widehat{\mathcal{E}}(u)[v]-(\mathrm{D} \widehat{\mathcal{E}}(u), u)(v, u)}{\|v\|} \\
& =\|\mathrm{D} \widehat{\mathcal{E}}(u)-(\mathrm{D} \widehat{\mathcal{E}}(u), u) u\|,
\end{aligned}
$$


where the second inequality follows by writing every $v \in V \backslash\{0\}$ as $v=(v, u) u+w$, and noting that $(w, u)=(v, u)-(v, u)(u, u)=0$. On the other hand, for $u \in$ $\operatorname{dom}(\mathcal{E})$ and $v \in X$ one has $(\mathcal{E}(u)-\mathcal{E}(v))^{+}>0$ if and only if $v \in X \cap V$. Hence, if $\mathrm{D} \widehat{\mathcal{E}}(u) \in H$ we estimate

$$
\begin{aligned}
\frac{(\mathcal{E}(u)-\mathcal{E}(v))^{+}}{\|v-u\|} \leq & \frac{(\mathrm{D} \widehat{\mathcal{E}}(u)[v-u])^{+}}{\|v-u\|} \\
\leq & \frac{(\mathrm{D} \widehat{\mathcal{E}}(u)[v-u]-(\mathrm{D} \widehat{\mathcal{E}}(u), u)(u, v-u))^{+}}{\|v-u\|} \\
& +\frac{((\mathrm{D} \widehat{\mathcal{E}}(u), u)(u, v-u))^{+}}{\|v-u\|} \\
\leq & \|\mathrm{D} \widehat{\mathcal{E}}(u)-(\mathrm{D} \widehat{\mathcal{E}}(u), u) u\| \\
& +(\mathrm{D} \widehat{\mathcal{E}}(u), u)^{+} \frac{1-(u, v)}{\sqrt{2(1-(u, v))}}
\end{aligned}
$$

the first inequality due to the convexity of $\widehat{\mathcal{E}}$ and the last one to the identity $\| v-$ $u \|^{2}=2(1-(u, v))$, since $\|u\|=\|v\|=1$. We take the limsup of (3.21) as $\|v-u\| \rightarrow 0$ and conclude the converse inequality of (3.20). Hence, the formula for $|\partial \mathcal{E}|$ ensues. Using this, it is easy to check that the map $u \mapsto|\partial \mathcal{E}|(u)$ is lower semicontinuous, whence (3.19).

Lemma 3.7. Under the above assumptions, the functional $\mathcal{E}$ defined by (3.18) complies with the chain rule of Definition 2.5.

Proof. Let us point out that any curve $u \in A C(0, T ; X)$ is a.e. differentiable with values in $H$, so that

$$
\left|u^{\prime}\right|(t)=\left\|u^{\prime}(t)\right\| \quad \text { for a.e. } t \in(0, T) .
$$

Now, if $u$ fulfills (2.28), necessarily $u(t) \in V$ (whence $\mathcal{E}(u(t))=\widehat{\mathcal{E}}(u(t)))$ for all $t \in[0, T])$. Besides, the fact that $|\partial \mathcal{E}|(u(t))<+\infty$ for a.e. $t \in(0, T)$ yields by Lemma 3.6 that $\mathrm{D} \widehat{\mathcal{E}}(u(t)) \in H$ for a.e. $t \in(0, T)$. Since $\widehat{\mathcal{E}}$ is smooth on $H$, it satisfies the chain rule

$$
\frac{d}{d t} \mathcal{E}(u(t))=\frac{d}{d t} \widehat{\mathcal{E}}(u(t))=\left(u^{\prime}(t), \mathrm{D} \widehat{\mathcal{E}}(u(t))\right) \quad \text { for a.e. } t \in(0, T) .
$$

The constraint $\|u(t)\|=1$ for all $t \in[0, T]$ implies that

$$
\left(u^{\prime}(t), u(t)\right)=0 \quad \text { for a.e. } t \in(0, T) .
$$


Hence, recalling (3.19) and (3.22), we deduce that

$$
\begin{array}{r}
\frac{d}{d t} \widehat{\mathcal{E}}(u(t))=\left(u^{\prime}(t), \mathrm{D} \widehat{\mathcal{E}}(u(t))-(\mathrm{D} \widehat{\mathcal{E}}(u(t)), u(t)) u(t)\right) \geq \\
\text { for a.e. } t \in(0, T)
\end{array}
$$

namely the chain rule inequality (2.29).

Hence, the metric formulation (2.31)-(2.32) associated with the energy functional $\mathcal{E}$ (3.18) and with the quadratic dissipation $\psi(r):=\frac{1}{2} r^{2}$ for all $r \geq 0$ reads

$$
\begin{gathered}
\text { find } u \in \operatorname{AC}(0, T ; X) \text { s.t. the map } t \in(0, T) \mapsto \mathcal{E}(u(t)) \\
\text { is absolutely continuous, and } \\
\frac{d}{d t} \mathcal{E}(u(t)) \leq-\frac{1}{2}\left\|u^{\prime}(t)\right\|^{2}-\frac{1}{2}\|\mathrm{D} \widehat{\mathcal{E}}(u(t))-(\mathrm{D} \widehat{\mathcal{E}}(u(t)), u(t)) u(t)\|^{2} \\
\text { for a.e. } t \in(0, T) .
\end{gathered}
$$

In view of (3.17), $\mathcal{E}$ complies with the assumptions of our main Theorem 3.5, providing existence and approximation of a solution $u \in \operatorname{AC}(0, T ; X)$ to the Cauchy problem for (3.25) for any initial datum $u_{0} \in V \cap X$. It follows from the related energy identity (3.9) that $u$ has the further regularity $u \in H^{1}(0, T ; H) \cap L^{\infty}(0, T ; V)$. In fact, using the chain rule (3.23), (3.24), the energy identity (3.9) and the CauchySchwarz inequality we deduce that $u$ solves the gradient flow equation

$$
\left\{\begin{array}{ll}
u^{\prime}(t)=-(\mathrm{D} \widehat{\mathcal{E}}(u(t))-(\mathrm{D} \widehat{\mathcal{E}}(u(t)), u(t)) u(t)) & \text { for a.e. } t \in(0, T), \\
\|u(t)\|=1 & \forall t \in[0, T]
\end{array} .\right.
$$

\section{Proof of the main result}

\subsection{Estimates for the $(\psi)$-Moreau-Yosida approximation}

In this section we collect some general properties of the time-incremental problem (3.2). Namely, given $r>0, \mathrm{t} \in(0, T)$, and $u \in X$, we study the minimization problem

$$
\inf _{v \in X}\left\{r \psi\left(\frac{\Delta(u, v)}{r}\right)+\mathcal{E}_{\mathrm{t}+r}(v)\right\} .
$$

Note that, in the case $\Delta$ is a distance on $X, \psi(x):=x^{2} / 2$, and the functional $\mathcal{E}$ does not depend on $t,(4.1)$ actually reduces to

$$
\inf _{v \in X}\left\{\frac{d^{2}(v, u)}{r}+\mathcal{E}(v)\right\}
$$


which is related to the Moreau-Yosida approximation of $\mathcal{E}$. The properties of the minimization problem (4.2) have been thoroughly studied in [5] (see also [45]). In fact, Lemmas 4.4 and 4.5 below are an extension to our framework of analogous results contained in [5, Chapter 3]. Besides the time dependence of the energy functionals, one of the main difficulties here is given by the general choice of the function $\psi$ : indeed, we neither assume that $\psi$ is strictly convex, nor that it is everywhere differentiable or even everywhere finite on $[0,+\infty)$.

Definition 4.1 ( $\psi$-Moreau-Yosida approximation). For $r>0$, we consider

$$
\mathrm{E}_{\mathrm{t}, r}(u ; v):=r \psi\left(\frac{\Delta(u, v)}{r}\right)+\mathcal{E}_{\mathrm{t}+r}(v),
$$

and define the $\psi$-Moreau-Yosida approximation $\mathscr{E}$ t, $r$ of the functionals $\mathcal{E}$ by

$$
\mathscr{E} t, r(u):=\inf _{v \in X} \mathrm{E}_{\mathrm{t}, r}(u ; v) .
$$

We also denote by $J_{r}(\mathrm{t}, u)$ the set where the infimum in (4.3) is attained, i.e.

$$
J_{\mathrm{t}, r}(u):=\underset{v \in X}{\operatorname{Argmin}} \mathrm{E}_{\mathrm{t}, r}(u ; v) .
$$

Remark 4.2 (Simplifying assumptions). By adding a positive constant to $\mathcal{E}_{t}$, we can always assume that $C_{0}=-1$ in $(2.19 \mathrm{~b})$, i.e.

$$
\inf _{t \in[0, T], v \in \mathrm{D}} \mathcal{E}_{t}(v) \geq 1
$$

we can therefore set

$$
\mathcal{F}(v):=\Delta\left(x_{o}, v\right)+\mathcal{E}_{0}(v)
$$

with

$$
\begin{gathered}
\frac{1}{A} \sup _{t \in[0, T]}\left(\Delta\left(x_{o}, v\right)+\mathcal{E}_{t}(v)\right) \leq \mathcal{F}(v) \leq A \inf _{t \in[0, T]}\left(\Delta\left(x_{o}, v\right)+\mathcal{E}_{t}(v)\right) \quad \forall v \in \mathrm{D}, \\
\left|\partial_{t} \mathcal{E}_{t}(v)\right| \leq A \mathcal{F}(v) \quad \forall v \in \mathrm{D}, \\
\mathcal{E}_{t}(v) \leq\left(\mathcal{E}_{s}(v)+C_{1} \Delta\left(x_{o}, v\right)|t-s|\right) \exp \left(C_{1}|t-s|\right) \quad \forall t, s \in[0, T], v \in \mathrm{D},
\end{gathered}
$$

for a suitable constant $A>0$ (all inequalities being trivial if $v \in \mathrm{D} \backslash D_{0}$ ).

Remark 4.3 (Elementary properties of $\psi$ ). Being 0 a minimum point for $\psi$, it is immediate to check that $\psi$ is non decreasing on $\operatorname{dom}(\psi)$. We denote by $\psi_{-}^{\prime}$ and $\psi_{+}^{\prime}$ respectively the (non decreasing) left and right derivatives of $\psi$ on $D(\psi)$ (we set $\left.\psi_{+}^{\prime}(a)=+\infty\right)$, which satisfy

$$
\psi_{-}^{\prime}(x) \leq \psi_{+}^{\prime}(x), \quad \partial \psi(x)=\left[\psi_{-}^{\prime}(x), \psi_{+}^{\prime}(x)\right] \quad \forall x \in \operatorname{dom}(\psi) .
$$

Since $\psi$ has a superlinear growth, the conjugate function $\psi^{*}$ is finite at each $y \in$ $[0,+\infty)$, non decreasing, and satisfies $\psi^{*}(0)=0$. 
The following result collects some properties of $\mathscr{E} t, r$.

Lemma 4.4. Under the same assumptions as in Theorem 3.5 and Remark 4.2, there exists a constant $C>0$ such that for every $\mathrm{t} \in[0, T], u \in D_{0}$ there holds

$$
\mathcal{F}\left(u_{r}\right) \leq C \mathcal{F}(u) \quad \forall 0<r \leq \min (1, T-\mathrm{t}), u_{r} \in J_{\mathrm{t}, r}(u),
$$

and

$$
\begin{aligned}
\mathscr{E} \mathrm{t}, r_{2} & (u)-C \mathcal{F}(u) r_{2} \leq \mathscr{E}_{\mathrm{t}, r_{1}}(u)-C \mathcal{F}(u) r_{1} \leq \mathcal{E}_{\mathrm{t}}(u) \\
& \text { for every } 0<r_{1}<r_{2} \leq \min (1, T-\mathrm{t}) ;
\end{aligned}
$$

in particular, the map $r \mapsto \mathscr{E}_{t, r}(u)$ is a linear perturbation of a non increasing function and has bounded variation. Moreover,

$$
\lim _{r \downarrow 0} \sup _{u_{r} \in J_{\mathrm{t}, r}(u)} \Delta\left(u, u_{r}\right)=0, \quad \lim _{r \downarrow 0} \mathscr{E}_{\mathrm{t}, r}(u)=\mathcal{E}_{\mathrm{t}}(u),
$$

and

$$
\left|\partial \mathcal{E}_{\mathrm{t}+r}\right|\left(u_{r}\right) \leq \psi_{+}^{\prime}\left(\frac{\Delta\left(u, u_{r}\right)}{r}\right) \quad \text { if } u_{r} \in J_{\mathrm{t}, r}(u) \text { and } 0 \leq \frac{\Delta\left(u, u_{r}\right)}{r}<a .
$$

Proof.

Step 1: proof of (4.11). First of all, let us point out that the minimality of $u_{r}$ and (4.7) yield

$$
r \psi\left(\frac{\Delta\left(u, u_{r}\right)}{r}\right) \leq \mathcal{E}_{\mathrm{t}+r}(u) \leq A \mathcal{F}(u) \quad \forall u_{r} \in J_{\mathrm{t}, r}(u) .
$$

Let us now fix $\rho>0$ so that $\psi^{*}(\rho)<1 / 2$ and therefore, being $r \leq 1$,

$$
r \psi\left(\frac{\Delta\left(u, u_{r}\right)}{r}\right) \geq \rho \Delta\left(u, u_{r}\right)-r \psi^{*}(\rho) \geq \rho \Delta\left(u, u_{r}\right)-1 / 2 ;
$$

by the minimality of $u_{r}$ we thus get for $C:=2 A\left(1+\rho^{-1}\right)$

$$
\begin{aligned}
\mathcal{F}\left(u_{r}\right) & \leq C\left(\rho \Delta\left(u, u_{r}\right)-1 / 2+\mathcal{E}_{\mathrm{t}+r}\left(u_{r}\right)\right) \leq C\left(r \psi\left(\frac{\Delta\left(u, u_{r}\right)}{r}\right)+\mathcal{E}_{\mathrm{t}+r}\left(u_{r}\right)\right) \\
& \leq C \mathcal{E}_{\mathrm{t}+r}(u) \leq C A \mathcal{F}(u),
\end{aligned}
$$

the latter two passages following from (4.15).

Step 2: proof of (4.12). We first observe that, for every $0<r_{1}<r_{2}$

$$
\begin{aligned}
\mathrm{E}_{\mathrm{t}, r_{2}}(u ; v)-\mathrm{E}_{\mathrm{t}, r_{1}}(u ; v) \leq & r_{2} \psi\left(\frac{\Delta(u, v)}{r_{2}}\right)-r_{1} \psi\left(\frac{\Delta(u, v)}{r_{1}}\right) \\
& +\int_{r_{1}}^{r_{2}} \partial_{t} \mathcal{E}_{\mathrm{t}+\theta}(v) \mathrm{d} \theta \\
\leq & A\left(r_{2}-r_{1}\right) \mathcal{F}(v) \quad \forall v \in \mathrm{D},
\end{aligned}
$$


the last passage due to (4.8) and the fact that the map $r \mapsto r \psi(x / r)$ is non increasing. Choosing $v=u_{r_{1}}$ and recalling that $\mathscr{E}_{\mathrm{t}, r_{2}}(u) \leq \mathrm{E}_{\mathrm{t}, r_{2}}\left(u ; u_{r_{1}}\right)$ we get by (4.11)

$$
\mathscr{E} \mathrm{t}, r_{2}(u)-\mathscr{E} \mathrm{t}, r_{1}(u) \leq A\left(r_{2}-r_{1}\right) \mathcal{F}\left(u_{r_{1}}\right) \leq C A\left(r_{2}-r_{1}\right) \mathcal{F}(u)
$$

Step 3: proof of (4.13). From (4.15) and the definition of $\psi^{*}$ we get

$$
M \Delta\left(u, u_{r}\right) \leq A \mathcal{F}(u)+r \psi^{*}(M) \quad \forall M>0, \forall r \leq T-\mathrm{t}, \forall u_{r} \in J_{\mathrm{t}, r}(u) .
$$

Taking the supremum with respect to $u_{r}$ and the lim sup as $r \downarrow 0$ we get

$$
M \limsup \sup _{r \downarrow 0} \Delta\left(u, u_{r}\right) \leq A \mathcal{F}(u) \quad \forall M>0,
$$

yielding the first limit in (4.13).

To check the second one, we note that, by (4.12), (4.13), (2.20), and the lower semicontinuity of the functional $u \mapsto \mathcal{E}_{\mathrm{t}}(u)$,

$$
\mathcal{E}_{\mathrm{t}}(u) \geq \limsup _{r \downarrow 0} \mathscr{E}, r(u) \geq \liminf _{r \downarrow 0} \mathcal{E}_{\mathrm{t}+r}\left(u_{r}\right) \geq \mathcal{E}_{\mathrm{t}}(u)
$$

Step 4: proof of (4.14). We may assume $a<+\infty$, the case $a=+\infty$ being easier to handle. Hence, we fix $r>0, u_{r} \in J_{r}(\mathrm{t}, u)$ such that $\Delta\left(u, u_{r}\right)<a$, and for simplicity we set $\mathcal{E}(u):=\mathcal{E}_{\mathrm{t}+r}(u)$. We also suppose that $|\partial \mathcal{E}|\left(u_{r}\right)>0$ : otherwise, the inequality would be trivial. Note that,

$$
|\partial \mathcal{E}|\left(u_{r}\right)=\limsup _{\Delta\left(u_{r}, v\right) \rightarrow 0} \frac{\left(\mathcal{E}\left(u_{r}\right)-\mathcal{E}(v)\right)^{+}}{\Delta\left(u_{r}, v\right)}=\limsup _{\Delta\left(u_{r}, v\right) \rightarrow 0, \Delta(u, v)>\Delta\left(u, u_{r}\right)} \frac{\left(\mathcal{E}\left(u_{r}\right)-\mathcal{E}(v)\right)^{+}}{\Delta\left(u_{r}, v\right)} .
$$

Indeed, since $|\partial \mathcal{E}|\left(u_{r}\right)>0$, there exists a sequence $\left\{v_{k}\right\}$ with $\Delta\left(u_{r}, v_{k}\right) \rightarrow 0$ as $k \uparrow \infty$ and $\bar{k} \in \mathbb{N}$ such that

$$
|\partial \mathcal{E}|\left(u_{r}\right)=\lim _{k \uparrow+\infty} \frac{\left(\mathcal{E}\left(u_{r}\right)-\mathcal{E}\left(v_{k}\right)\right)^{+}}{\Delta\left(u_{r}, v_{k}\right)} \text {, with } \mathcal{E}\left(v_{k}\right)<\mathcal{E}\left(u_{r}\right), \frac{\Delta\left(u, v_{k}\right)}{r}<a,
$$

for $k \geq \bar{k}$, the latter inequality following from the fact that $\Delta\left(u, u_{r}\right)<a$ and $\Delta\left(u_{r}, v_{k}\right) \rightarrow 0$. Hence, $\Delta\left(u, v_{k}\right)>\Delta\left(u, u_{r}\right)$ for all $k \geq \bar{k}$ : otherwise, if $\Delta\left(u, v_{k}\right) \leq$ $\Delta\left(u, u_{r}\right)$, the minimization (4.1) would lead to

$$
\mathcal{E}\left(u_{r}\right)+r \psi\left(\frac{\Delta\left(u, u_{r}\right)}{r}\right) \leq \mathcal{E}\left(v_{k}\right)+r \psi\left(\frac{\Delta\left(u, v_{k}\right)}{r}\right) \leq \mathcal{E}\left(v_{k}\right)+r \psi\left(\frac{\Delta\left(u, u_{r}\right)}{r}\right)
$$


(the second passage by the monotonicity of $\psi$ ), contrary to (4.18). Furthermore, for every $k \geq \bar{k}$

$$
\begin{aligned}
\frac{\mathcal{E}\left(u_{r}\right)-\mathcal{E}\left(v_{k}\right)}{\Delta\left(u_{r}, v_{k}\right)} & \leq r \frac{\psi\left(\frac{\Delta\left(u, v_{k}\right)}{r}\right)-\psi\left(\frac{\Delta\left(u, u_{r}\right)}{r}\right)}{\Delta\left(u_{r}, v_{k}\right)} \\
& \leq \frac{\psi\left(\frac{\Delta\left(u, v_{k}\right)}{r}\right)-\psi\left(\frac{\Delta\left(u, u_{r}\right)}{r}\right)}{\frac{\Delta\left(u, v_{k}\right)}{r}-\frac{\Delta\left(u, u_{r}\right)}{r}}
\end{aligned}
$$

the first inequality following from (4.1), and the second one by the triangle inequality. Therefore, noting that $0<\Delta\left(u, v_{k}\right)-\Delta\left(u, u_{r}\right) \leq \Delta\left(u_{r}, v_{k}\right) \rightarrow 0$, we have

$$
\begin{aligned}
|\partial \mathcal{E}|\left(u_{r}\right) & =\limsup _{\Delta\left(u_{r}, v_{k}\right) \rightarrow 0} \frac{\left(\mathcal{E}\left(u_{r}\right)-\mathcal{E}\left(v_{k}\right)\right)^{+}}{\Delta\left(u_{r}, v_{k}\right)} \\
& \leq \liminf _{\Delta\left(u_{r}, v_{k}\right) \rightarrow 0} \frac{\psi\left(\frac{\Delta\left(u, v_{k}\right)}{r}\right)-\psi\left(\frac{\Delta\left(u, u_{r}\right)}{r}\right)}{\frac{\Delta\left(u, v_{k}\right)}{r}-\frac{\Delta\left(u, u_{r}\right)}{r}} \\
& \leq \limsup _{h \downarrow 0} \frac{\psi\left(\frac{\Delta\left(u, u_{r}\right)}{r}+h\right)-\psi\left(\frac{\Delta\left(u, u_{r}\right)}{r}\right)}{h}=\psi_{+}^{\prime}\left(\frac{\Delta\left(u, u_{r}\right)}{r}\right) .
\end{aligned}
$$

Before proving the following lemma, which will play a crucial role later on, we recall for the reader's convenience the well-known duality formula relating $\psi$ and $\psi^{*}$

$$
\psi^{*}(y)=y x-\psi(x) \quad \forall y \in\left[\psi_{-}^{\prime}(x), \psi_{+}^{\prime}(x)\right] .
$$

Moreover, in the case in which $D(\psi)=[0, a]$, with $a<+\infty$, and $\ell:=\psi_{-}^{\prime}(a)<$ $+\infty$, there holds

$$
\begin{array}{ll}
\psi^{*}(y) \leq \psi^{*}(\ell)=\ell a-\psi(a) & \forall y \leq \ell, \\
\psi^{*}(y)=y a-\psi(a)=\psi^{*}(\ell)+(y-\ell) a & \forall y>\ell .
\end{array}
$$

Indeed, the first inequality follows from the monotonicity of $\psi^{*}$; on the other hand, recalling that $\partial \psi(a)=[\ell,+\infty)$ and that $\partial \psi^{*}=(\partial \psi)^{-1}$, we conclude that $\partial \psi^{*}(y)=\{a\}$ for $y>\ell$, whence the second of (4.20).

Lemma 4.5. Under the same assumptions as in Theorem 3.5, for every $\mathrm{t} \in[0, T)$, $u \in D_{0}$, and for a.e. $r>0$

$$
\begin{aligned}
& \text { the map } r \mapsto \mathscr{E}_{\mathrm{t}, r}(u) \text { is differentiable, } \\
& \psi_{-}^{\prime}\left(\Delta\left(u, u_{r}\right) / r\right)<+\infty \text { at each point } u_{r} \in J_{\mathrm{t}, r}(u),
\end{aligned}
$$


and

$$
\begin{aligned}
\frac{d}{d r} \mathscr{E}_{\mathrm{t}, r}(u) & \leq \partial_{t} \mathcal{E}_{\mathrm{t}+r}\left(u_{r}\right)-\psi^{*}\left(\psi_{-}^{\prime}\left(\frac{\Delta\left(u, u_{r}\right)}{r}\right)\right), \\
\frac{d}{d r} \mathscr{E}_{\mathrm{t}, r}(u) & \leq \partial_{t} \mathcal{E}_{\mathrm{t}+r}\left(u_{r}\right)-\psi^{*}\left(\left|\partial \mathcal{E}_{\mathrm{t}+r}\right|\left(u_{r}\right)\right),
\end{aligned}
$$

where we adopt the convention of writing $\psi_{-}^{\prime}(0)=0$. In particular, we have

$$
\begin{aligned}
r_{0} \psi\left(\frac{\Delta\left(u, u_{r_{0}}\right)}{r_{0}}\right) & +\int_{0}^{r_{0}} \psi^{*}\left(\left|\partial \mathcal{E}_{\mathrm{t}+r}\right|\left(u_{r}\right)\right) \mathrm{d} r+\mathcal{E}_{\mathrm{t}+r_{0}}\left(u_{r_{0}}\right) \\
& \leq \mathcal{E}_{\mathrm{t}}(u)+\int_{0}^{r_{0}} \partial_{t} \mathcal{E}_{\mathrm{t}+r}\left(u_{r}\right) \mathrm{d} r
\end{aligned}
$$

for every $0<r_{0} \leq T-\mathrm{t}$ and $u_{r_{0}} \in J_{\mathrm{t}, r_{0}}(u)$.

Proof. Preliminarily, let us point out that (4.16) yields for any $r_{1}, r_{2}>0$

$$
\begin{aligned}
\mathscr{E}_{\mathrm{t}, r_{2}}(u)-\mathscr{E}_{\mathrm{t}, r_{1}}(u) \leq & r_{2} \psi\left(\frac{\Delta\left(u, u_{r_{1}}\right)}{r_{2}}\right)-r_{1} \psi\left(\frac{\Delta\left(u, u_{r_{1}}\right)}{r_{1}}\right) \\
& +\left(\mathcal{E}_{\mathrm{t}+r_{2}}\left(u_{r_{1}}\right)-\mathcal{E}_{\mathrm{t}+r_{1}}\left(u_{r_{1}}\right)\right) .
\end{aligned}
$$

Step 1: proof of (4.22). Since by (4.12) the map $r \mapsto \mathscr{E} t, r(u)$ is a linear perturbation of a monotone map, it is also almost everywhere differentiable on $(0,+\infty)$; let $r$ be a point of differentiability and let us choose $r_{1}:=r$ and $r_{2}:=r+h, h>0$, (so that $\Delta\left(u, u_{r}\right) /(r+h) \in D(\psi)$ as well) in (4.25); we also set

$G(h):=\mathscr{E} \mathfrak{E}, r+h(u)-\mathcal{E}_{\mathrm{t}+r+h}\left(u_{r}\right)$ so that $\frac{d}{d s} \mathscr{E} \mathfrak{t},\left.s(u)\right|_{s=r}=\left.\frac{d}{d h} G(h)\right|_{h=0}-\partial_{t} \mathcal{E}_{\mathrm{t}+r}\left(u_{r}\right)$.

When $\Delta\left(u, u_{r}\right)>0(4.25)$ yields, with easy calculations,

$$
\begin{aligned}
\frac{G(h)-G(0)}{h} & \leq \frac{1}{h}\left((r+h) \psi\left(\frac{\Delta\left(u, u_{r}\right)}{r+h}\right)-r \psi\left(\frac{\Delta\left(u, u_{r}\right)}{r}\right)\right) \\
& \leq \psi\left(\frac{\Delta\left(u, u_{r}\right)}{r+h}\right)-\frac{\Delta\left(u, u_{r}\right)}{r+h}\left(\frac{\psi\left(\frac{\Delta\left(u, u_{r}\right)}{r+h}\right)-\psi\left(\frac{\Delta\left(u, u_{r}\right)}{r}\right)}{\frac{\Delta\left(u, u_{r}\right)}{r+h}-\frac{\Delta\left(u, u_{r}\right)}{r}}\right) .
\end{aligned}
$$

Letting $h \downarrow 0$, also taking into account that $\psi$ is continuous on $\operatorname{int}(\operatorname{dom}(\psi))$ we have

$$
\frac{\psi\left(\frac{\Delta\left(u, u_{r}\right)}{r+h}\right)-\psi\left(\frac{\Delta\left(u, u_{r}\right)}{r}\right)}{\frac{\Delta\left(u, u_{r}\right)}{r+h}-\frac{\Delta\left(u, u_{r}\right)}{r}} \uparrow \psi_{-}^{\prime}\left(\frac{\Delta\left(u, u_{r}\right)}{r}\right) \quad \text { as } h \downarrow 0 .
$$


Therefore, we infer that

$$
G^{\prime}(0) \leq \psi\left(\frac{\Delta\left(u, u_{r}\right)}{r}\right)-\frac{\Delta\left(u, u_{r}\right)}{r} \psi_{-}^{\prime}\left(\frac{\Delta\left(u, u_{r}\right)}{r}\right)=-\psi^{*}\left(\psi_{-}^{\prime}\left(\frac{\Delta\left(u, u_{r}\right)}{r}\right)\right)
$$

and the same relation holds even when $\Delta\left(u, u_{r}\right)=0$, by the convention $\psi_{-}^{\prime}(0)=0$.

Step 2: proof of (4.23). If $\Delta\left(u, u_{r}\right)=0$, then we may note that $\psi^{*}\left(\psi_{+}^{\prime}\left(\Delta\left(u, u_{r}\right) / r\right)\right)=$ $\psi^{*}\left(\psi_{+}^{\prime}(0)\right)=-\psi(0)=0$, so that (4.23) follows from (4.14), the monotonicity of $\psi^{*}$, and (4.22).

The same argument shows that (4.23) is an immediate consequence of (4.14) and (4.22) when $\partial \psi$ is single valued, since in that case $\psi_{-}^{\prime}=\psi_{+}^{\prime}$.

In order to prove (4.23) in the general case, we can assume without loss of generality that $\left|\partial \mathcal{E}_{\mathrm{t}+r}\right|\left(u_{r}\right)>0$, and that

$$
\left|\partial \mathcal{E}_{\mathrm{t}+r}\right|\left(u_{r}\right) \geq \psi_{-}^{\prime}\left(\frac{\Delta\left(u, u_{r}\right)}{r}\right)
$$

If not, we would trivially conclude (4.23) from (4.22). On the other hand, by (4.14)

$$
\left|\partial \mathcal{E}_{\mathrm{t}+r}\right|\left(u_{r}\right) \leq \psi_{+}^{\prime}\left(\frac{\Delta\left(u, u_{r}\right)}{r}\right), \quad \text { thus } \quad\left|\partial \mathcal{E}_{\mathrm{t}+r}\right|\left(u_{r}\right) \in \partial \psi\left(\frac{\Delta\left(u, u_{r}\right)}{r}\right)
$$

Let us denote by $x$ the number $\frac{\Delta\left(u, u_{r}\right)}{r}$ : arguing as in the proof of Lemma 4.4, we can select a sequence $\left\{v_{k}\right\}$ fulfilling

$$
\begin{aligned}
& \Delta\left(u_{r}, v_{k}\right) \rightarrow 0 \text { as } k \uparrow \infty, \quad \Delta\left(u, u_{r}\right)<\Delta\left(u, v_{k}\right), \\
& \left|\partial \mathcal{E}_{\mathrm{t}+r}\right|\left(u_{r}\right)=\lim _{k \uparrow \infty} \frac{\mathcal{E}_{\mathrm{t}+r}\left(u_{r}\right)-\mathcal{E}_{\mathrm{t}+r}\left(v_{k}\right)}{\Delta\left(u_{r}, v_{k}\right)} .
\end{aligned}
$$

We also set

$$
r_{k}:=\frac{\Delta\left(u, v_{k}\right)}{x}>\frac{\Delta\left(u, u_{r}\right)}{x}=r
$$

noting that

$r_{k} \downarrow r$ as $k \uparrow \infty$, and

$$
\limsup _{k \uparrow \infty} \frac{\mathcal{E}_{\mathrm{t}+r}\left(u_{r}\right)-\mathcal{E}_{\mathrm{t}+r}\left(v_{k}\right)}{\Delta\left(u_{r}, v_{k}\right)} \leq \limsup _{\Delta\left(u_{r}, v_{k}\right) \rightarrow 0} \frac{\mathcal{E}_{\mathrm{t}+r}\left(u_{r}\right)-\mathcal{E}_{\mathrm{t}+r}\left(v_{k}\right)}{x\left(r_{k}-r\right)}
$$


by the triangle inequality. Therefore,

$$
\begin{aligned}
& \left.\frac{d}{d s} \mathscr{E}_{\mathrm{t}, s}(u)\right|_{s=r}=\lim _{k \uparrow \infty} \frac{\mathscr{E}_{\mathrm{t}, r_{k}}(u)-\mathscr{E}_{\mathrm{t}, r}(u)}{r_{k}-r} \\
& \leq \liminf _{k \uparrow \infty} \frac{1}{r_{k}-r}\left(\mathcal{E}_{\mathrm{t}+r_{k}}\left(v_{k}\right)+r_{k} \psi\left(\frac{\Delta\left(u, v_{k}\right)}{r_{k}}\right)-\mathcal{E}_{\mathrm{t}+r}\left(u_{r}\right)-r \psi\left(\frac{\Delta\left(u, u_{r}\right)}{r}\right)\right) \\
& =\liminf _{k \uparrow \infty} \frac{1}{r_{k}-r}\left(\psi(x)\left(r_{k}-r\right)+\left(\mathcal{E}_{\mathrm{t}+r_{k}}\left(v_{k}\right)-\mathcal{E}_{\mathrm{t}+r}\left(v_{k}\right)\right)+\left(\mathcal{E}_{\mathrm{t}+r}\left(v_{k}\right)-\mathcal{E}_{\mathrm{t}+r}\left(u_{r}\right)\right)\right) \\
& \leq \psi(x)+\limsup _{k \uparrow \infty} \frac{\mathcal{E}_{\mathrm{t}+r_{k}}\left(v_{k}\right)-\mathcal{E}_{\mathrm{t}+r}\left(v_{k}\right)}{r_{k}-r}-\lim _{k \uparrow \infty} \frac{\mathcal{E}_{\mathrm{t}+r}\left(u_{r}\right)-\mathcal{E}_{\mathrm{t}+r}\left(v_{k}\right)}{r_{k}-r} \\
& \leq \partial_{t} \mathcal{E}_{\mathrm{t}+r}\left(u_{r}\right)+\psi(x)-x\left|\partial \mathcal{E}_{\mathrm{t}+r}\right|\left(u_{r}\right)=\partial_{t} \mathcal{E}_{\mathrm{t}+r}\left(u_{r}\right)-\psi^{*}\left(\left|\partial \mathcal{E}_{\mathrm{t}+r}\right|\left(u_{r}\right)\right), \quad(4.30)
\end{aligned}
$$

where the fifth passage follows from (2.36), (4.28), and (4.29), whereas the last identity is due to (4.19) and (4.27).

We argue analogously in the case in which int $(\operatorname{dom}(\psi))=(0, a)$, with $a<$ $+\infty$, and $\Delta\left(u, u_{r}\right) / r=a$. Indeed, we repeat (4.28)-(4.30), the only difference being that the final identity in (4.30) follows now from (4.20).

Step 3: proof of (4.24). We note that for every $r_{0}>0$ and every (measurable) selection $u_{r} \in J_{r}(\mathrm{t}, u), r \in\left(0, r_{0}\right]$,

$$
\begin{aligned}
\psi\left(\frac{\Delta\left(u, u_{r_{0}}\right)}{r_{0}}\right)+\mathcal{E}_{\mathrm{t}+r_{0}}\left(u_{r_{0}}\right)-\mathcal{E}_{\mathrm{t}}(u) & =\mathscr{E}_{\mathrm{t}, r_{0}}(u)-\lim _{r \downarrow 0} \mathscr{E}_{\mathrm{t}, r}(u) \leq \int_{0}^{r_{0}} \frac{d}{d r} \mathscr{E}_{\mathrm{t}, r}(u) \mathrm{d} r \\
& \leq \int_{0}^{r_{0}}\left(\partial_{t} \mathcal{E}_{\mathrm{t}+r}\left(u_{r}\right)-\psi^{*}\left(\left|\partial \mathcal{E}_{\mathrm{t}+r}\right|\left(u_{r}\right)\right)\right) \mathrm{d} r,
\end{aligned}
$$

where we have used (4.13) in the first passage, the monotonicity (up to a linear perturbation) of $r \mapsto \mathscr{E}_{\mathrm{t}, r}(u)$ in the second passage and, finally, (4.23).

\subsection{Estimates for the approximate solutions}

\section{A priori estimates}

Preliminarily, we recall the following well-known Discrete Gronwall Lemma:

Lemma 4.6. Let $B, b$, and $\kappa$ be positive constants with $1-b \geq \frac{1}{\kappa}>0$ and let $\left\{a_{n}\right\} \subset[0,+\infty)$ be a sequence satisfying

$$
a_{n} \leq B+b \sum_{k=1}^{n} a_{k} \quad \forall n \in \mathbb{N} .
$$

Then, $\left\{a_{n}\right\}$ can be bounded by

$$
a_{n} \leq \kappa B e^{\kappa b n} \quad \forall n \in \mathbb{N} .
$$


Proposition 4.7 (A priori estimates). Under the assumptions of Theorem 3.5, for $\tau>0$ let $\left\{U_{\tau}^{n}\right\}_{n=1}^{N}$ be a family of solutions to (3.2), and let $\bar{U}_{\tau}, \underline{U}_{\tau}$, and $\widetilde{U}_{\tau}$ be the interpolants defined by (3.3) and (3.5). Then, the discrete energy inequality

$$
\begin{gathered}
\int_{\mathbf{s}_{\tau}}^{\mathbf{t}_{\tau}} \psi\left(\frac{\Delta\left(\underline{U}_{\tau}(r), \bar{U}_{\tau}(r)\right)}{\tau}\right) \mathrm{d} r+\int_{\mathbf{s}_{\tau}}^{\mathbf{t}_{\tau}} \psi^{*}\left(\left|\partial \mathcal{E}_{r}\right|\left(\widetilde{U}_{\tau}(r)\right)\right) \mathrm{d} r+\mathcal{E}_{\mathrm{t}_{\tau}}\left(\bar{U}_{\tau}\left(\mathbf{t}_{\tau}\right)\right) \\
\leq \mathcal{E}_{\mathbf{s}_{\tau}}\left(\bar{U}_{\tau}\left(\mathbf{s}_{\tau}\right)\right)+\int_{\mathbf{s}_{\tau}}^{\mathbf{t}_{\tau}} \partial_{t} \mathcal{E}_{r}\left(\widetilde{U}_{\tau}(r)\right) \mathrm{d} r
\end{gathered}
$$

holds for every pair of nodes $\mathbf{S}_{\tau}<\mathbf{t}_{\tau} \in \mathscr{P}_{\tau}$. Moreover, there exists a positive constant $C$ such that the following estimates hold for every $\tau>0$ :

$$
\begin{aligned}
& \int_{0}^{T} \psi\left(\frac{\Delta\left(\underline{U}_{\tau}(r), \bar{U}_{\tau}(r)\right)}{\tau}\right) \mathrm{d} r \leq C, \quad \int_{0}^{T} \psi^{*}\left(\left|\partial \mathcal{E}_{r}\right|\left(\widetilde{U}_{\tau}(r)\right)\right) \mathrm{d} r \leq C, \\
& \mathcal{F}\left(\bar{U}_{\tau}(t)\right) \leq C, \quad \mathcal{F}\left(\widetilde{U}_{\tau}(t)\right) \leq C \quad \forall t \in(0, T), \\
& \sup _{t \in(0, T)} \Delta\left(\underline{U}_{\tau}(t), \widetilde{U}_{\tau}(t)\right)=o(1), \sup _{t \in(0, T)} \Delta\left(\underline{U}_{\tau}(t), \bar{U}_{\tau}(t)\right)=o(1) \text { as } \tau \downarrow 0 .
\end{aligned}
$$

Proof. Let $t_{j-1}, t_{j}$ be two consecutive nodes of the partition $\mathscr{P}_{\tau}(c f$. (3.1)), and let $t \in\left(t_{j-1}, t_{j}\right]$ : referring to the definition (4.3) of the Moreau-Yosida approximation $\mathscr{E}_{\mathrm{t}, r}$, let us apply inequality (4.24) with the choices $\mathrm{t}=t_{j-1}, u=U_{\tau}^{j-1}, r_{0}=$ $t-t_{j-1}, u_{r_{0}}=\widetilde{U}_{\tau}(t), u_{r}=\widetilde{U}_{\tau}(r)$ for $r \in\left(t_{j-1}, t\right)$. Thus, after changing variable in the two integrals we obtain

$$
\begin{gathered}
\left(t-t_{j-1}\right) \psi\left(\frac{\Delta\left(U_{\tau}^{j-1}, \widetilde{U}_{\tau}(t)\right)}{t-t_{j-1}}\right)+\int_{t_{j-1}}^{t} \psi^{*}\left(\left|\partial \mathcal{E}_{r}\right|\left(\widetilde{U}_{\tau}(r)\right)\right) \mathrm{d} r+\mathcal{E}_{t}\left(\widetilde{U}_{\tau}(t)\right) \\
\leq \mathcal{E}_{t_{j-1}}\left(U_{\tau}^{j-1}\right)+\int_{t_{j-1}}^{t} \partial_{t} \mathcal{E}_{r}\left(\widetilde{U}_{\tau}(r)\right) \mathrm{d} r \quad \forall t \in\left(t_{j-1}, t_{j}\right] .
\end{gathered}
$$

Writing (4.36) for $t=t_{j}$, we obtain

$$
\begin{gathered}
\int_{t_{j-1}}^{t_{j}} \psi\left(\frac{\Delta\left(\underline{U}_{\tau}(r), \bar{U}_{\tau}(r)\right)}{\tau}\right) \mathrm{d} r+\int_{t_{j-1}}^{t_{j}} \psi^{*}\left(\left|\partial \mathcal{E}_{r}\right|\left(\widetilde{U}_{\tau}(r)\right)\right) \mathrm{d} r+\mathcal{E}_{t_{j}}\left(U_{\tau}^{j}\right) \\
\leq \mathcal{E}_{t_{j-1}}\left(U_{\tau}^{j-1}\right)+\int_{t_{j-1}}^{t_{j}} \partial_{t} \mathcal{E}_{r}\left(\widetilde{U}_{\tau}(r)\right) \mathrm{d} r .
\end{gathered}
$$

Hence, (4.32) follows by adding up the contributions (4.37) on the subintervals of the partition. It follows from the superlinear growth of $\psi$ that there exists a positive 
constant $C$ such that

$$
\begin{aligned}
\int_{t_{j-1}}^{t_{j}} \psi\left(\frac{\Delta\left(\underline{U}_{\tau}(r), \bar{U}_{\tau}(r)\right)}{\tau}\right) \mathrm{d} r & \geq \Delta\left(U_{\tau}^{j-1}, U_{\tau}^{j}\right)-C \tau \\
& \geq \Delta\left(x_{o}, U_{\tau}^{j}\right)-\Delta\left(x_{o}, U_{\tau}^{j-1}\right)-C \tau,
\end{aligned}
$$

the last passage following from the triangle inequality. Combining (4.37) and (4.38), recalling that $\psi^{*}$ is positive ( $c f$. Remark 4.3), summing over the index $j$, and using (4.7), we obtain

$$
\begin{aligned}
\frac{1}{A} \mathcal{F}\left(U_{\tau}^{j}\right) & \leq \Delta\left(x_{o}, U_{\tau}^{j}\right)+\mathcal{E}_{t_{j}}\left(U_{\tau}^{j}\right) \\
& \leq C T+\Delta\left(x_{o}, u_{0}\right)+\mathcal{E}_{0}\left(u_{0}\right)+\int_{0}^{t_{j}} \partial_{t} \mathcal{E}_{r}\left(\widetilde{U}_{\tau}(r)\right) \mathrm{d} r \\
& \leq C T+\Delta\left(x_{o}, u_{0}\right)+\mathcal{E}_{0}\left(u_{0}\right)+A \int_{0}^{t_{j}} \mathcal{F}\left(\widetilde{U}_{\tau}(r)\right) \mathrm{d} r \\
& \leq C T+\Delta\left(x_{o}, u_{0}\right)+\mathcal{E}_{0}\left(u_{0}\right)+A C \int_{0}^{t_{j}} \mathcal{F}\left(\underline{U}_{\tau}(r)\right) \mathrm{d} r
\end{aligned}
$$

the third inequality following from (4.8) and the fourth one from (4.11). Therefore, we deduce that

$$
\mathcal{F}\left(U_{\tau}^{j}\right) \leq c_{0}+C \sum_{k=1}^{j} \tau \mathcal{F}\left(U_{\tau}^{k}\right) \quad \forall j=1, \ldots, N,
$$

where the constant $c_{0}$ only depends on the initial and the problem data. Then, the discrete Gronwall Lemma 4.6 yields the first estimate in (4.34), and the second one readily follows thanks to (4.11). Recalling (4.8), we also infer that

$$
\int_{0}^{t_{j}}\left|\partial_{t} \mathcal{E}_{r}\left(\tilde{U}_{\tau}(r)\right)\right| \mathrm{d} r \leq C \quad \text { for all } \tau>0
$$

Therefore, summing up over the index $j$ and arguing by comparison in (4.37) we conclude the estimates of (4.33).

Finally, in order to check the first limit in (4.35) (in fact, the second one in (4.35) can be proved in the same way), we start by noting that, from (4.36), the positivity of $\psi^{*}$ and the previous estimates

$$
\left(t-t_{j-1}\right) \psi\left(\frac{\Delta\left(U_{\tau}^{j-1}, \tilde{U}_{\tau}(t)\right)}{t-t_{j-1}}\right) \leq C \quad \forall t \in\left(t_{j-1}, t_{j}\right], j=1, \ldots N .
$$

Combining this with the superlinear growth of $\psi$ we obtain that for any $M \geq 0$ there exists $S \geq 0$ fulfilling

$$
\Delta\left(\underline{U}_{\tau}(t), \tilde{U}_{\tau}(t)\right) \leq \frac{C}{M}+\left(t-t_{j-1}\right) \frac{S}{M} \leq \frac{C}{M}+\tau \frac{S}{M} \quad \forall t \in\left(t_{j-1}, t_{j}\right], \forall j=1, \ldots, N .
$$


Thus, we easily deduce that for any $\varepsilon>0$ there exist $\tau_{0}>0$ such that for $0<\tau<\tau_{0}$

$$
\Delta\left(\underline{U}_{\tau}(t), \widetilde{U}_{\tau}(t)\right) \leq \varepsilon \quad \forall t \in(0, T),
$$

whence the desired conclusion.

\subsection{Passage to the limit and proof of existence}

The proof of the ensuing Proposition 4.9 is an adaptation of the argument developed for [5, Corollary 3.3.4], and is based on the following version of the Ascoli-Arzelà compactness theorem, proved in [5, Proposition 3.3.1], which for convenience we recall here, in a slightly simplified form.

Proposition 4.8. Under assumptions (2.1)-(2.2) on the space $X$, let $\mathcal{K}$ be a $\sigma$ sequentially compact subset of $X$ with the following property: for all $\left\{u_{n}\right\},\left\{v_{n}\right\}, u$, $v \in \mathcal{K}$,

$$
\left(u_{n}, v_{n}\right) \stackrel{\sigma}{\rightarrow}(u, v) \Rightarrow \liminf _{n \uparrow \infty} \Delta\left(u_{n}, v_{n}\right) \geq \Delta(u, v) .
$$

Let $\left\{u_{n}\right\}$ be a sequence of curves $u_{n}:[0, T] \rightarrow X$ fulfilling

$$
\begin{gathered}
u_{n}(t) \in \mathcal{K} \quad \forall n \in N, \quad \forall t \in[0, T], \\
\limsup _{n \uparrow} \Delta\left(u_{n}(s), u_{n}(t)\right) \leq \omega(s, t) \quad \forall s, t \in[0, T], s<t,
\end{gathered}
$$

where $\omega:[0, T] \times[0, T] \rightarrow[0,+\infty)$ is a function such that

$$
\lim _{s \uparrow r, t \uparrow r s<t} \omega(s, t)=0 \quad \forall r \in[0, T] .
$$

Then, there exist an increasing subsequence $k \mapsto n_{k}$ and a $\sigma$-continuous curve $u:[0, T] \rightarrow X$ such that

$$
u_{n_{k}}(t) \stackrel{\sigma}{\rightarrow} u(t) \quad \forall t \in[0, T],
$$

and the limit curve $u$ satisfies

$$
\lim _{r \uparrow s} \Delta(u(r), u(s))=\lim _{t \downarrow s} \Delta(u(s), u(t))=0 \quad \forall s \in(0, T) .
$$

Proposition 4.9 (Compactness of the approximate solutions). Under the assumptions of Theorem 3.5, given any vanishing sequence $\tau_{n} \downarrow 0$ of time steps, there exist a subsequence (still labeled $\tau_{n}$ ), a limit curve $u \in \mathrm{AC}\left(0, T ; X_{0}\right)$, and a function $L \in L^{1}(0, T)$ such that the following convergences hold as $n \uparrow \infty$

$$
\begin{aligned}
& \bar{U}_{\tau_{n}}(t) \stackrel{\sigma}{\rightarrow} u(t), \quad \underline{U}_{\tau_{n}}(t) \stackrel{\sigma}{\rightarrow} u(t), \quad \tilde{U}_{\tau_{n}}(t) \stackrel{\sigma}{\rightarrow} u(t) \quad \forall t \in[0, T], \\
& \liminf _{n \uparrow \infty} \mathcal{E}_{t}\left(\widetilde{U}_{\tau_{n}}(t)\right) \geq \mathcal{E}_{t}(u(t)), \liminf _{n \uparrow \infty} \mathcal{E}_{\overline{\mathrm{t}}_{\tau_{n}}(t)}\left(\bar{U}_{\tau_{n}}(t)\right) \geq \mathcal{E}_{t}(u(t)) \forall t \in[0, T], \\
& \frac{\Delta\left(\underline{U}_{\tau_{n}}, \bar{U}_{\tau_{n}}\right)}{\tau_{n}} \rightarrow L \quad \text { in } L^{1}(0, T), \quad L(t) \geq\left|u^{\prime}\right|(t) \quad \text { for a.e. } t \in(0, T), \\
& \liminf _{n \uparrow \infty}\left|\partial \mathcal{E}_{t}\right|\left(\widetilde{U}_{\tau_{n}}(t)\right) \geq\left|\partial^{-} \mathcal{E}_{t}\right|(u(t)) \\
& \forall t \in[0, T] .
\end{aligned}
$$


Proof. It is easy to see that estimate (4.33) and the superlinear growth of $\psi$ entail that the family

$$
\left\{\frac{\Delta\left(\underline{U}_{\tau}(r), \bar{U}_{\tau}(r)\right)}{\tau}\right\}_{\tau} \text { is bounded and uniformly integrable in } L^{1}(0, T) .
$$

Therefore, the Dunford-Pettis criterion ensures that it is weakly relatively compact in $L^{1}(0, T)$, whence the first of (4.47).

Exploiting (4.34) and assumption (2.38) on the sublevels of $\mathcal{F}$, we can apply Proposition 4.8 to the sequence $\left\{\bar{U}_{\tau_{n}}\right\}$, of course with $\mathcal{K}$ as a suitable sublevel of $\mathcal{F}$ (note that (4.41) is then a consequence of (2.34)). Moreover, in order to check (4.43), let us note that, by the triangle inequality,

$$
\Delta\left(\bar{U}_{\tau_{n}}(s), \bar{U}_{\tau_{n}}(t)\right) \leq \int_{\overline{\mathrm{t}}_{\tau_{n}}(s)}^{\overline{\mathrm{t}}_{\tau_{n}}(t)} \frac{\Delta\left(\underline{U}_{\tau_{n}}(r), \bar{U}_{\tau_{n}}(r)\right)}{\tau_{n}} \mathrm{~d} r \quad \forall 0 \leq s \leq t \leq T .
$$

Therefore, passing to the limit as $n \uparrow \infty$ and recalling (2.34), (3.4) and (4.47), we infer

$$
\Delta(u(s), u(t)) \leq \limsup _{n \uparrow \infty} \Delta\left(\bar{U}_{\tau_{n}}(s), \bar{U}_{\tau_{n}}(t)\right) \leq \int_{s}^{t} L(r) \mathrm{d} r \quad \forall 0 \leq s \leq t \leq T,
$$

whence (4.43).

Thus, thanks to Proposition 4.8 we find a limit curve $u \in \mathrm{C}^{0}([0, T] ; X)$ and a subsequence along which

$$
\bar{U}_{\tau_{n}}(t) \stackrel{\sigma}{\rightarrow} u(t) \quad \forall t \in[0, T] .
$$

Convergences (4.45) for $\underline{U}_{\tau_{n}}$ and $\widetilde{U}_{\tau_{n}}$ then follow from (4.35) and (2.39); further, $u \in \mathrm{AC}\left(0, T ; X_{0}\right)$ by (4.49). The second inequality in (4.47) also follows from (4.49) and Proposition 2.2.

As far as (4.46) is concerned, the first liminf inequality ensues from (4.45), estimate (4.34) and the lower semicontinuity assumption (2.34); in the same way, from (2.35) we deduce that

$$
\underset{n \uparrow \infty}{\limsup } \partial_{t} \mathcal{E}_{t}\left(\widetilde{U}_{\tau_{n}}(t)\right) \leq \partial_{t} \mathcal{E}_{t}(u(t)) \quad \forall t \in[0, T]
$$

Finally, in order to prove the second of (4.46), we combine the estimate

$$
\begin{aligned}
\left|\mathcal{E}_{\overline{\mathrm{t}}_{\tau_{n}}(t)}\left(\bar{U}_{\tau_{n}}(t)\right)-\mathcal{E}_{t}\left(\bar{U}_{\tau_{n}}(t)\right)\right| & \leq \int_{t}^{\overline{\mathrm{t}}_{\tau_{n}}(t)} \partial_{t} \mathcal{E}_{r}\left(\bar{U}_{\tau_{n}}(t)\right) \mathrm{d} r \\
& \leq A \int_{t}^{\overline{\mathrm{t}}_{\tau_{n}}(t)} \mathcal{F}\left(\bar{U}_{\tau_{n}}(t)\right) \mathrm{d} r \\
& \leq C\left(\overline{\mathrm{t}}_{\tau_{n}}(t)-t\right) \quad \forall t \in[0, T]
\end{aligned}
$$


with the lower semicontinuity inequality (again due to (2.34))

$$
\liminf _{n \uparrow \infty} \mathcal{E}_{t}\left(\bar{U}_{\tau_{n}}(t)\right) \geq \mathcal{E}_{t}(u(t)) \quad \forall t \in[0, T] .
$$

In the end, (4.48) follows from (4.45) and the definition (2.18) of $\left|\partial^{-} \mathcal{E}\right|$.

We may now complete the proof of Theorem 3.5.

Proof of Theorem 3.5. By (4.45), $u(0)=u_{0}$. Let us fix $t \in(0, T]$ and consider inequality (4.32) for the nodes $\overline{\mathbf{t}}_{\tau_{n}}(t)$ and $\mathbf{S}=0$ :

$$
\begin{aligned}
\int_{0}^{\bar{\tau}_{\tau_{n}}(t)} \psi\left(\frac{\Delta\left(\underline{U}_{\tau_{n}}(r), \bar{U}_{\tau_{n}}(r)\right)}{\tau_{n}}\right) \mathrm{d} r & +\int_{0}^{\bar{\tau}_{\tau_{n}}(t)} \psi^{*}\left(\left|\partial \mathcal{E}_{r}\right|\left(\widetilde{U}_{\tau_{n}}(r)\right)\right) \mathrm{d} r \\
& +\mathcal{E}_{\overline{\mathrm{\tau}}_{\tau_{n}}(t)}\left(\bar{U}_{\tau_{n}}(t)\right) \\
& \leq \mathcal{E}_{0}\left(u_{0}\right)+\int_{0}^{\overline{\mathrm{t}}_{\tau_{n}}(t)} \partial_{t} \mathcal{E}_{r}\left(\widetilde{U}_{\tau_{n}}(r)\right) \mathrm{d} r .
\end{aligned}
$$

We have

$$
\begin{aligned}
\liminf _{n \uparrow \infty}\left(\int_{0}^{\bar{\tau}_{\tau_{n}}(t)} \psi\left(\frac{\Delta\left(\underline{U}_{\tau_{n}}(r), \bar{U}_{\tau_{n}}(r)\right)}{\tau_{n}}\right) \mathrm{d} r\right) & \geq \int_{0}^{t} \psi(L(r)) \mathrm{d} r \\
& \geq \int_{0}^{t} \psi\left(\left|u^{\prime}\right|(r)\right) \mathrm{d} r,
\end{aligned}
$$

the first inequality due to the first of (4.47) and the convexity of $\psi$, while the second inequality follows from the second of (4.47) and the monotonicity of $\psi$. Now, for later convenience let us set $A(t):=\liminf _{n \uparrow \infty}\left|\partial \mathcal{E}_{t}\right|\left(\widetilde{U}_{\tau_{n}}(t)\right)$ for a.e. $t \in(0, T)$. By Fatou's lemma and the monotonicity of $\psi^{*}$ we have

$$
\begin{aligned}
\liminf _{n \uparrow \infty}\left(\int_{0}^{\bar{\tau}_{\tau_{n}}(t)} \psi^{*}\left(\left|\partial \mathcal{E}_{r}\right|\left(\widetilde{U}_{\tau_{n}}(r)\right)\right) \mathrm{d} r\right) & \geq \int_{0}^{\bar{\tau}_{\tau_{n}}(t)} \liminf _{n \uparrow \infty} \psi^{*}\left(\left|\partial \mathcal{E}_{r}\right|\left(\widetilde{U}_{\tau_{n}}(r)\right)\right) \mathrm{d} r \\
& \geq \int_{0}^{t} \psi^{*}(A(r)) \mathrm{d} r \\
& \geq \int_{0}^{t} \psi^{*}\left(\left|\partial^{-} \mathcal{E}_{r}\right|(u(r))\right) \mathrm{d} r .
\end{aligned}
$$

Furthermore, (4.52), (4.53), and the a priori estimates (4.33) also entail

$$
\begin{aligned}
\int_{0}^{T}\left|u^{\prime}\right|(r)\left|\partial^{-} \mathcal{E}_{r}\right|(u(r)) \mathrm{d} r \leq & \int_{0}^{T} \psi\left(\left|u^{\prime}\right|(r)\right) \mathrm{d} r \\
& +\int_{0}^{T} \psi^{*}\left(\left|\partial^{-} \mathcal{E}_{r}\right|(u(r))\right) \mathrm{d} r \leq C .
\end{aligned}
$$


In the same way, we find that

$$
\mathcal{E}_{t}(u(t))+\int_{0}^{T}\left|\partial_{t} \mathcal{E}_{t}(u(t))\right| \mathrm{d} t \leq C \quad \forall t \in[0, T] .
$$

Now, combining (4.45), (4.46), (4.50), (4.52)-(4.53), and exploiting the Fatou lemma, we manage to pass to the limit in (4.51) and obtain

$$
\begin{aligned}
\int_{0}^{t} \psi\left(\left|u^{\prime}\right|(r)\right) \mathrm{d} r & +\int_{0}^{t} \psi^{*}\left(\left|\partial^{-} \mathcal{E}_{r}\right|(u(r))\right) \mathrm{d} r+\mathcal{E}_{t}(u(t)) \\
& \leq \mathcal{E}_{0}\left(u_{0}\right)+\int_{0}^{t} \partial_{t} \mathcal{E}_{r}(u(r)) \mathrm{d} r
\end{aligned}
$$

On the other hand, note that, thanks to (4.54) and (4.55), we may apply the chain rule of Definition 2.5 to the limit curve $u \in \mathrm{AC}\left(0, T ; X_{0}\right)$. Upon integration, we get

$$
\begin{aligned}
\mathcal{E}_{0}\left(u_{0}\right)- & \mathcal{E}_{t}(u(t))+\int_{0}^{t} \partial_{t} \mathcal{E}_{r}(u(r)) \mathrm{d} r \leq \int_{0}^{t}\left|u^{\prime}\right|(r)\left|\partial^{-} \mathcal{E}_{r}\right|(u(r)) \mathrm{d} r \\
& \leq \int_{0}^{t} \psi\left(\left|u^{\prime}\right|(r)\right) \mathrm{d} r+\int_{0}^{t} \psi^{*}\left(\left|\partial^{-} \mathcal{E}_{r}\right|(u(r))\right) \mathrm{d} r .
\end{aligned}
$$

Thus, (4.56) yields

$$
\int_{0}^{t}\left(\psi\left(\left|u^{\prime}\right|(r)\right)+\psi^{*}\left(\left|\partial^{-} \mathcal{E}_{r}\right|(u(r))\right)+\frac{d}{d r} \mathcal{E}_{r}(u(r))-\partial_{t} \mathcal{E}_{r}(u(r))\right) \mathrm{d} r=0 .
$$

Since the integrand is non negative by inequality (2.29) and $t \in(0, T)$ is arbitrary, we deduce

$$
\begin{array}{r}
\psi\left(\left|u^{\prime}\right|(t)\right)+\psi^{*}\left(\left|\partial^{-} \mathcal{E}_{t}\right|(u(t))\right)+\frac{d}{d t} \mathcal{E}_{t}(u(t))-\partial_{t} \mathcal{E}_{t}(u(t))=0 \\
\text { for a.e. } t \in(0, T)
\end{array}
$$

i.e., (3.8), whence (2.33) as well. The above relation yields the energy identity (3.9) upon integration.

Finally, taking the lim sup as $n \uparrow \infty$ of (4.51) and again using the identity just proved, we deduce

$$
\begin{array}{r}
\limsup _{n \uparrow \infty}\left(\int_{0}^{\overline{\mathrm{t}}_{\tau_{n}}(t)} \psi\left(\frac{\Delta\left(\underline{U}_{\tau_{n}}(r), \bar{U}_{\tau_{n}}(r)\right)}{\tau_{n}}\right) \mathrm{d} r+\int_{0}^{\overline{\mathrm{t}}_{\tau_{n}}(t)} \psi^{*}\left(\left|\partial \mathcal{E}_{r}\right|\left(\widetilde{U}_{\tau_{n}}(r)\right)\right) \mathrm{d} r\right. \\
\left.\quad+\mathcal{E}_{\overline{\mathrm{t}}_{\tau_{n}}(t)}\left(\bar{U}_{\tau_{n}}(t)\right)\right) \\
\leq \mathcal{E}_{0}\left(u_{0}\right)+\int_{0}^{t} \partial_{t} \mathcal{E}_{r}(u(r)) \mathrm{d} r \\
=\int_{0}^{t} \psi\left(\left|u^{\prime}\right|(r)\right) \mathrm{d} r+\int_{0}^{t} \psi^{*}\left(\left|\partial^{-} \mathcal{E}_{r}\right|(u(r))\right) \mathrm{d} r+\mathcal{E}_{t}(u(t)) .
\end{array}
$$


So, taking into account (4.46), (4.52), and (4.53) and arguing by comparison, we deduce convergences (3.10)-(3.11) and the first of (3.12). We also conclude the second of (3.12) by taking the lim sup as $n \uparrow \infty$ of the following inequality

$$
\begin{array}{r}
\int_{0}^{\mathrm{t}_{\tau_{n}}(t)} \psi\left(\frac{\Delta\left(\underline{U}_{\tau_{n}}(r), \bar{U}_{\tau_{n}}(r)\right)}{\tau_{n}}\right) \mathrm{d} r+\int_{0}^{t} \psi^{*}\left(\left|\partial \mathcal{E}_{r}\right|\left(\widetilde{U}_{\tau_{n}}(r)\right)\right) \mathrm{d} r+\mathcal{E}_{t}\left(\widetilde{U}_{\tau_{n}}(t)\right) \\
\leq \mathcal{E}_{0}\left(u_{0}\right)+\int_{0}^{\bar{\tau}_{\tau_{n}}(t)} \partial_{t} \mathcal{E}_{r}\left(\widetilde{U}_{\tau_{n}}(r)\right) \mathrm{d} r
\end{array}
$$

(which is obtained by summing up (4.32) and (4.36)), and arguing as in the above lines.

In order to prove (3.13), we note that, combining (4.53) with (3.11) leads to

$$
\begin{aligned}
\lim _{n \uparrow \infty} \int_{0}^{t} \psi^{*}\left(\left|\partial \mathcal{E}_{r}\right|\left(\widetilde{U}_{\tau_{n}}(r)\right)\right) \mathrm{d} r & =\int_{0}^{t} \liminf _{n \uparrow \infty} \psi^{*}\left(\left|\partial \mathcal{E}_{r}\right|\left(\widetilde{U}_{\tau_{n}}(r)\right)\right) \mathrm{d} r \\
& =\int_{0}^{t} \psi^{*}(A(r)) \mathrm{d} r \\
& =\int_{0}^{t} \psi^{*}\left(\left|\partial^{-} \mathcal{E}_{r}\right|(u(r))\right) \mathrm{d} r \quad \forall t \in[0, T],
\end{aligned}
$$

whence

$$
\liminf _{n \uparrow \infty} \psi^{*}\left(\left|\partial \mathcal{E}_{t}\right|\left(\widetilde{U}_{\tau_{n}}(t)\right)\right)=\psi^{*}(A(t))=\psi^{*}\left(\left|\partial^{-} \mathcal{E}_{t}\right|(u(t))\right) \quad \text { for a.e. } t \in(0, T) .
$$

Recalling (2.33), we conclude that for a.e. $t \in(0, T)$

$$
\begin{aligned}
\left|u^{\prime}\right|(t) \cdot A(t) & \leq \psi\left(\left|u^{\prime}\right|(t)\right)+\psi^{*}(A(t)) \\
& =\left|u^{\prime}\right|(t) \cdot\left|\partial^{-} \mathcal{E}_{t}\right|(u(t)) \leq\left|u^{\prime}\right|(t) \cdot A(t)
\end{aligned}
$$

so that all the above inequalities hold as equalities and, if $\left|u^{\prime}\right|(t) \neq 0$, we conclude $A(t)=\left|\partial^{-} \mathcal{E}_{t}\right|(u(t))$, while $\left|u^{\prime}\right|(t)=0$ leads to the second of (3.13).

Finally, suppose first that $\psi^{*}$ has superlinear growth at infinity: it follows from the a priori estimate (4.33) that the sequence $\left\{\left|\partial \mathcal{E}_{t}\right|\left(\widetilde{U}_{\tau_{n}}\right)\right\}$ is uniformly integrable in $L^{1}(0, T)$. Hence, the fundamental compactness theorem of Young measures theory (see [10, Theorem 1] and also [12]) ensures that $\left\{\left|\partial \mathcal{E}_{t}\right|\left(\widetilde{U}_{\tau_{n}}\right)\right\}$ admits a subsequence (which we do not relabel) and a limit Young measure $\boldsymbol{v}=\left\{v_{t}\right\}_{t \in(0, T)}$ ( $v_{t}$ being a probability measure on $\mathbb{R}$ for a.e. $t \in(0, T))$ such that

$$
v_{t} \text { is concentrated on the set } \mathscr{L}(t)
$$

of the limit points of $\left\{\left|\partial \mathcal{E}_{t}\right|\left(\widetilde{U}_{\tau_{n}}\right)(t)\right\}$ for a.e. $t \in(0, T)$,

$$
\left|\partial \mathcal{E}_{t}\right|\left(\widetilde{U}_{\tau_{n}}\right) \rightarrow \Sigma(t):=\int_{\mathbb{R}} \xi \mathrm{d} v_{t}(\xi) \quad \text { in } L^{1}((0, T)),
$$

$$
\liminf _{n \uparrow \infty} \int_{0}^{T} \psi^{*}\left(\left|\partial \mathcal{E}_{r}\right|\left(\widetilde{U}_{\tau_{n}}\right)(r)\right) \mathrm{d} r \geq \int_{0}^{T}\left(\int_{\mathbb{R}} \psi^{*}(\xi) \mathrm{d} v_{r}(\xi)\right) \mathrm{d} r
$$


Now, the definition of $\left|\partial^{-} \mathcal{E}\right|$ gives for a.e. $t \in(0, T)$

$$
\left|\partial^{-} \mathcal{E}_{t}\right|(u(t)) \leq \xi, \quad \text { whence } \quad \psi^{*}\left(\left|\partial^{-} \mathcal{E}_{t}\right|(u(t))\right) \leq \psi^{*}(\xi) \quad \forall \xi \in \mathscr{L}(t) .
$$

Combining the above inequality, (4.58), (4.60), and (4.62), we deduce that

$$
\int_{0}^{T}\left(\int_{\mathbb{R}} \psi^{*}(\xi) \mathrm{d} v_{r}(\xi)\right) \mathrm{d} r=\int_{0}^{T} \psi^{*}\left(\left|\partial^{-} \mathcal{E}_{r}\right|(u(r))\right) \mathrm{d} r,
$$

whence, again by (4.63),

$$
\psi^{*}(\xi)=\psi^{*}\left(\left|\partial^{-} \mathcal{E}_{t}\right|(u(t))\right) \quad \text { for a.e. } \xi \in \mathscr{L}(t) \text { for a.e. } t \in(0, T) .
$$

Taking into account the above identity, (2.33) and the first of (4.63) we thus conclude the following chain of inequalities

$$
\begin{aligned}
\left|u^{\prime}\right|(t) \xi & \leq \psi\left(\left|u^{\prime}\right|(t)\right)+\psi^{*}(\xi)=\psi\left(\left|u^{\prime}\right|(t)\right)+\psi^{*}\left(\left|\partial^{-} \mathcal{E}_{t}\right|(u(t))\right) \\
& =\left|u^{\prime}\right|(t)\left|\partial^{-} \mathcal{E}_{t}\right|(u(t)) \leq\left|u^{\prime}\right|(t) \xi \quad \forall \xi \in \mathscr{L}(t) \quad \text { for a.e. } t \in(0, T) .
\end{aligned}
$$

Then, recalling the definition (3.14) of the set $\mathscr{I}$, we conclude that

$$
\xi=\left|\partial^{-} \mathcal{E}_{t}\right|(u(t)) \quad \forall \xi \in \mathscr{L}(t) \quad \text { for a.e. } t \in \mathscr{I} .
$$

Therefore, the limit Young measure $\boldsymbol{v}$ is such that for a.e. $t \in \mathscr{I}$ its disintegration $v_{t}$ coincides with the Dirac mass $\delta_{\mid \partial-} \mathcal{E}_{t} \mid(u(t))$. Since the sequence $\left\{\left|\partial \mathcal{E}_{t}\right|\left(\widetilde{U}_{\tau_{n}}\right)\right\}$ is uniformly integrable, we conclude (3.15).

Without the superlinear growth assumption on $\psi^{*}$, using a version of the Biting Lemma (see e.g. [50, Theorem 13]) we deduce that there is a sequence of Borel subsets $O_{n} \subset(0, T)$, decreasing to $\emptyset$, such that, denoting by $I_{n}$ the indicator function of the set $(0, T) \backslash O_{n}$ the sequence $\omega_{n}:=I_{n}\left|\partial \mathcal{E}_{t}\right|\left(\widetilde{U}_{\tau_{n}}\right)$ is uniformly integrable in $L^{1}(0, T)$. Thus, we apply [10, Theorem 1] to the sequence $\left\{\omega_{n}\right\}$, find an associated limit Young measure $\boldsymbol{\mu}=\left\{\mu_{t}\right\}_{t \in(0, T)}$, and conclude relations (4.60)-(4.62) for a (not relabeled) subsequence $\left\{\omega_{n}\right\}$. Since the sequence $O_{n} \downarrow \emptyset$, we may check that for a.e. $t \in(0, T)$ the set of the limit points of $\left\{\omega_{n}(t)\right\}$ coincides with the set of the limit points of $\left\{\left|\partial \mathcal{E}_{t}\right|\left(\widetilde{U}_{\tau_{n}}\right)(t)\right\}$, hence relations (4.63) hold as well. On the other hand,

$$
\begin{aligned}
\liminf _{n \uparrow \infty} \int_{0}^{T} \psi^{*}\left(\omega_{n}(r)\right) \mathrm{d} r & \leq \liminf _{n \uparrow \infty} \int_{0}^{T} \psi^{*}\left(\left|\partial \mathcal{E}_{r}\right|\left(\widetilde{U}_{\tau_{n}}\right)(r)\right) \mathrm{d} r \\
& =\int_{0}^{T} \psi^{*}\left(\left|\partial^{-} \mathcal{E}_{r}\right|(u(r))\right) \mathrm{d} r
\end{aligned}
$$

so that we similarly conclude (4.64) for the Young measure $\boldsymbol{\mu}$. Arguing exactly in the same way as above, we infer (3.16).

Hence, the proof is done. 


\section{Part II: applications in Banach spaces}

\section{Preliminaries in Banach spaces}

In this part, we focus our attention on the case in which the ambient space $X$ ( $c f$. (2.1)) is a separable Banach space: to stress this assumption, we shall indicate it with the letter $B$. We shall denote by $\|\cdot\|$ the norm of $B$, by $\|\cdot\|_{*}$ the norm on the dual space $B^{\prime}$ and by $\langle\cdot, \cdot\rangle$ the duality pairing between $B^{\prime}$ and $B$.

For simplicity, in the sequel we shall work with non symmetric distances $\Delta$ on $B$ taking values in $[0,+\infty)$. Furthermore, to fix ideas we shall suppose that

$$
\sigma \text { is the strong topology of } B \text {. }
$$

\subsection{Sublinear functionals}

Let us recall that a sublinear functional is a convex and positively homogeneous map $\eta: B \rightarrow[0,+\infty)$, thus satisfying the following conditions:

$$
\begin{gathered}
\eta(\lambda v)=\lambda \eta(v) \quad \forall \lambda \geq 0 \forall v \in B \\
\eta(v+w) \leq \eta(v)+\eta(w) \quad \forall v, w \in B .
\end{gathered}
$$

It is easy to check that $\eta(0)=0$ and it is well known that, among all the positively homogeneous maps satisfying (5.1a), condition (5.1b) is equivalent to the convexity of $\eta$. We also assume that there exists a positive constant $K$ such that

$$
K^{-1}\|v\| \leq \eta(v) \leq K\|v\| \quad \forall v \in B .
$$

The choice

$$
\Delta(u, v):=\eta(v-u) \quad \forall u, v \in B
$$

induces an asymmetric distance on $B$ which satisfies the properties of Section 2.1 and is metrically equivalent to the distance induced by the norm of $B$. Therefore, given a proper functional $\mathcal{E}: B \rightarrow(-\infty,+\infty]$ and a point $u \in \operatorname{dom}(\mathcal{E})$, we shall use the notation

$$
|\partial \mathcal{E}|(u)=\limsup _{v \rightarrow u} \frac{(\mathcal{E}(u)-\mathcal{E}(v))^{+}}{\Delta(u, v)},
$$

and accordingly consider the relaxed slope $\left|\partial^{-} \mathcal{E}\right|(2.18)$.

\section{Duality}

For any $u \in B$ we also introduce the dual functional $\eta_{*}:=B^{\prime} \rightarrow[0,+\infty)$ defined by

$$
\eta_{*}(\sigma):=\sup _{v \in B \backslash\{0\}} \frac{\langle\sigma, v\rangle}{\eta(v)}=\sup \{\langle\sigma, v\rangle: \eta(v)=1\} \quad \forall \sigma \in B^{\prime} \quad \forall u \in B .
$$


Note that if $\eta$ is a norm, then the related functional $\eta_{*}$ coincides with the corresponding dual norm of $B^{\prime}$. Further, (5.1c) implies, respectively,

$$
K^{-1}\|y\|_{*} \leq \eta_{*}(y) \leq K\|y\|_{*} \quad \forall y \in B^{\prime} .
$$

Let $\psi:[0,+\infty) \rightarrow[0,+\infty)$ be a positive, convex, and lower semicontinuous function. We define the functional $\Psi: B \rightarrow[0,+\infty)$ by

$$
\Psi(v):=\psi(\eta(v)) \quad \forall v \in B,
$$

and denote by $\partial \Psi$ its subdifferential and by $\Psi^{*}$ its Fenchel-Moreau conjugate

$$
\Psi^{*}(\sigma):=\sup _{v \in B}(\langle\sigma, v\rangle-\Psi(v)) .
$$

In the sequel, we shall need the following duality result.

Lemma 5.1. The conjugate of $\Psi$ is given by

$$
\Psi^{*}(\sigma):=\psi^{*}\left(\eta_{*}(\sigma)\right) \quad \forall \sigma \in B^{\prime} .
$$

Moreover, we have the following characterization of the subdifferential $\partial \Psi$ : for all $v \in B$

$$
\sigma \in \partial \Psi(v) \Longleftrightarrow\left(\eta_{*}(\sigma) \in \partial \psi(\eta(v)) \text { and } \eta_{*}(\sigma) \cdot \eta(v)=\langle\sigma, v\rangle\right) \text {. }
$$

Proof. It follows from the definition of $\eta_{*}$ that $\langle\sigma, v\rangle \leq \eta(v) \eta_{*}(\sigma)$ for all $v \in B$ and $\sigma \in B^{\prime}$. Hence, by the definition of $\Psi^{*}$ we have

$$
\Psi^{*}(\sigma) \leq \sup _{v \in B}\left(\eta(v) \cdot \eta_{*}(\sigma)-\psi(\eta(v))\right)=\sup _{r \geq 0}\left(r \eta_{*}(\sigma)-\psi(r)\right)=\psi^{*}\left(\eta_{*}(\sigma)\right) .
$$

On the other hand, for any $\sigma \in B^{\prime}$ we can find a sequence $\left\{v_{n}\right\}$ fulfilling $\eta\left(v_{n}\right)=1$, and $\eta_{*}(\sigma)=\lim _{n \uparrow \infty}\left\langle\sigma, v_{n}\right\rangle$. Then, for any $r \geq 0$

$$
\begin{aligned}
r \eta_{*}(\sigma)-\psi(r) & =\lim _{n \uparrow \infty}\left(\left\langle\sigma, r v_{n}\right\rangle-\psi\left(r \eta\left(v_{n}\right)\right)\right)=\lim _{n \uparrow \infty}\left(\left\langle\sigma, r v_{n}\right\rangle-\psi\left(\eta\left(r v_{n}\right)\right)\right) \\
& \leq \sup _{v \in B}(\langle\sigma, v\rangle-\psi(\eta(v)))=\Psi^{*}(\sigma),
\end{aligned}
$$

and (5.7) ensues.

Thanks to (5.7), it is straightforward to check that

$$
\left(\Psi(v)+\Psi^{*}(\sigma)=\langle\sigma, v\rangle\right) \Leftrightarrow\left(\psi(\eta(v))+\psi^{*}\left(\eta_{*}(\sigma)\right)=\eta(v) \cdot \eta_{*}(\sigma)=\langle\sigma, v\rangle\right) .
$$

On the other hand, the standard convex analysis characterization of the subdifferential in terms of the Legendre-Fenchel-Moreau transform yields

$$
\begin{gathered}
\sigma \in \partial \Psi(v) \Leftrightarrow \quad \Psi(v)+\Psi^{*}(\sigma)=\langle\sigma, v\rangle, \\
\eta_{*}(\sigma) \in \partial \psi(\eta(v)) \Leftrightarrow \psi(\eta(v))+\psi^{*}\left(\eta_{*}(\sigma)\right)=\eta(v) \cdot \eta_{*}(\sigma) .
\end{gathered}
$$

Combining the above relations, we readily deduce (5.8). 


\subsection{Subdifferential and slopes for admissible functionals}

Definition 5.2 (Fréchet subdifferential). Let $\mathcal{E}: B \rightarrow(-\infty,+\infty]$ be a proper functional; the Fréchet subdifferential $\partial \mathcal{E}(u) \subset B^{\prime}$ of $\mathcal{E}$ at a point $u \in \operatorname{dom}(\mathcal{E})$ is defined by

$$
\xi \in \partial \mathcal{E}(u) \quad \Leftrightarrow \quad \liminf _{w \rightarrow u} \frac{\mathcal{E}(w)-\mathcal{E}(u)-\langle\xi, w-u\rangle}{\|w-u\|} \geq 0 .
$$

It is well known that the subdifferential is single-valued and coincides with the usual differential $\mathrm{D} \mathcal{E}$ when it exists, e.g. if $\mathcal{E}$ is a functional of class $\mathrm{C}^{1}$. If $\mathcal{E}$ is convex, then $\partial \mathcal{E}$ can be equivalently characterized by

$$
\xi \in \partial \mathcal{E}(u) \Leftrightarrow \mathcal{E}(w)-\mathcal{E}(u) \geq\langle\xi, w-u\rangle \quad \forall w \in B,
$$

i.e. the Fréchet subdifferential coincides with the subdifferential in the sense of convex analysis. In fact, in the sequel we shall consider a more general convexity property.

\section{$\lambda$-convexity}

We recall that a proper functional $\mathcal{E}: B \rightarrow(-\infty,+\infty]$ is $\lambda$-convex for some $\lambda \in \mathbb{R}$ if

$$
\begin{aligned}
\mathcal{E}\left(u_{\theta}\right) & \leq(1-\theta) \mathcal{E}\left(u_{0}\right)+\theta \mathcal{E}\left(u_{1}\right)-\frac{1}{2} \lambda \theta(1-\theta)\left\|u_{0}-u_{1}\right\|^{2} \\
\forall \theta & \in[0,1] \quad \forall u_{0}, u_{1} \in B,
\end{aligned}
$$

where we have set $u_{\theta}=(1-\theta) u_{0}+\theta u_{1}$. The following result extends [5, Proposition 1.4.4, Theorem 2.4.9] to the asymmetric setting and to $\lambda$-convex functionals.

Lemma 5.3. Let $\mathcal{E}: B \rightarrow(-\infty,+\infty]$ be proper, lower semicontinuous, and $\lambda$ convex for some $\lambda \in \mathbb{R}$. Then,

1. for all $u \in \operatorname{dom}(\mathcal{E})$ the Fréchet subdifferential $\partial \mathcal{E}(u)$ is a convex weakly*-closed set, it can be characterized by

$$
\xi \in \partial \mathcal{E}(u) \Leftrightarrow \mathcal{E}(w)-\mathcal{E}(u) \geq\langle\xi, w-u\rangle+\frac{\lambda}{2}\|w-u\|^{2} \quad \forall w \in B,
$$

and the graph of the operator $\partial \mathcal{E}$ is strongly-weakly* closed, namely

$$
u_{n} \rightarrow u, \quad \xi_{n} \rightarrow^{*} \xi, \quad \xi_{n} \in \partial \mathcal{E}\left(u_{n}\right) \quad \Rightarrow \quad \xi \in \partial \mathcal{E}(u) .
$$

2. Let $\eta$ be a positively homogeneous functional fulfilling (5.1a,b,c) and inducing the asymmetric distance $\Delta$ (5.2); let $|\partial \mathcal{E}|$ be the $\Delta$-local slope of $\mathcal{E}$. Then,

$$
\begin{aligned}
& |\partial \mathcal{E}|(u)=\sup _{v \neq u}\left(\frac{\mathcal{E}(u)-\mathcal{E}(v)}{\eta(v-u)}-\frac{1}{2}|\lambda| K^{2} \eta(v-u)\right)^{+} \quad \forall u \in \operatorname{dom}(\mathcal{E}), \\
& |\partial \mathcal{E}|(u)=\min _{\xi \in \partial \mathcal{E}(u)} \eta_{*}(-\xi) \quad \forall u \in \operatorname{dom}(\mathcal{E}), \\
& \text { the map } u \mapsto|\partial \mathcal{E}|(u) \text { is lower semicontinuous. }
\end{aligned}
$$


Proof. Easy computations lead to (5.11), which in turn yields (5.12). Further, we note that (5.1c) and (5.10) yield that

$\mathcal{E}\left(u_{\theta}\right) \leq(1-\theta) \mathcal{E}\left(u_{0}\right)+\theta \mathcal{E}\left(u_{1}\right)+\frac{|\lambda|}{2} K^{2} \theta(1-\theta) \eta^{2}\left(u_{1}-u_{0}\right) \quad \forall \theta \in[0,1] \forall u_{0}, u_{1} \in B$.

Moving from the above inequality and repeating the very same computations as in the proof of [5, Theorem 2.4.9], one checks (5.13). Finally, (5.11) and (5.1c) again yield that for all $u \in \operatorname{dom}(\mathcal{E})$

$$
\mathcal{E}(u)-\mathcal{E}(u+w)-\frac{1}{2}|\lambda| K^{2} \eta^{2}(w) \leq\langle-\xi, w\rangle \quad \forall w \in B \quad \forall \xi \in \partial \mathcal{E}(u) .
$$

Taking into account (5.13), we deduce that

$$
|\partial \mathcal{E}|(u) \leq \min _{\xi \in \partial \mathcal{E}(u)} \eta_{*}(-\xi) .
$$

To prove the converse inequality, we introduce the quantity

$$
\delta \mathcal{E}(u ; w):=\limsup _{\varepsilon \downarrow 0} \frac{\mathcal{E}(u+\varepsilon w)-\mathcal{E}(u)}{\varepsilon} \quad \text { for } u \in \operatorname{dom}(\mathcal{E}), w \in B .
$$

Using the $\lambda$-convexity inequality (5.10) and the definition of $|\partial \mathcal{E}|$ it is not difficult to check that

$$
\begin{gathered}
\text { the map } w \mapsto \delta \mathcal{E}(u ; w) \text { is convex for all } u \in \operatorname{dom}(\mathcal{E}), \\
\left\{\begin{array}{l}
\mathcal{E}(u+w)-\mathcal{E}(u) \geq \delta \mathcal{E}(u ; w)+\frac{\lambda}{2}\|w\|^{2} \\
\delta \mathcal{E}(u ; w) \geq-|\partial \mathcal{E}|(u) \eta(w)
\end{array} \quad \forall u \in \operatorname{dom}(\mathcal{E}), w \in B .\right.
\end{gathered}
$$

Now, mimicking the proof of [5, Proposition 1.4.4], we consider the epigraph

$$
\mathcal{K}_{+}=\{(w, r) \in B \times \mathbb{R}: r \geq \delta \mathcal{E}(u ; w)\}
$$

of the function $w \mapsto \delta \mathcal{E}(u ; w)$ and the open hypograph

$$
\mathcal{K}_{-}=\{(w, r) \in B \times \mathbb{R}: r<-|\partial \mathcal{E}|(u) \eta(w)\}
$$

of $w \mapsto-|\partial \mathcal{E}|(u) \eta(w)$. Since $\mathcal{K}_{+}$and $\mathcal{K}_{-}$are disjoint by (5.16), a version of the Hahn-Banach theorem yields that there exists $\xi \in B^{\prime}$ and $\alpha \in \mathbb{R}$ such that

$$
-|\partial \mathcal{E}|(u) \eta(w) \leq\langle\xi, w\rangle+\alpha \leq \delta \mathcal{E}(u ; w) \quad \forall w \in B
$$

A standard argument shows that $\alpha=0$. Hence, from the first inequality in (5.17) and the arbitrariness of $w$ we deduce that $\eta_{*}(-\xi) \leq|\partial \mathcal{E}|(u)$. The second of (5.17), combined with (5.16), gives that $\xi$ fulfills (5.11). Thus, $\xi \in \partial \mathcal{E}(u)$, and (5.14) ensues. Finally, in order to check (5.15) we fix a sequence $u_{n} \rightarrow u$ with $\Lambda:=$ 
$\lim \inf _{n \uparrow \infty}|\partial \mathcal{E}|\left(u_{n}\right)<+\infty$. For any $\varepsilon>0$ there exists a subsequence $\left\{u_{n^{\prime}}\right\}$ and, accordingly, a sequence $\left\{\xi_{n^{\prime}}\right\} \subset B^{\prime}$, with $\xi_{n^{\prime}} \in \partial \mathcal{E}\left(u_{n^{\prime}}\right)$ for all $n^{\prime}$, fulfilling

$$
\lim _{n^{\prime} \uparrow \infty} \eta_{*}\left(-\xi_{n^{\prime}}\right)=\lim _{n^{\prime} \uparrow \infty} \min _{\xi \in \partial \mathcal{E}\left(u_{n^{\prime}}\right)} \eta_{*}(-\xi)=\lim _{n^{\prime} \uparrow \infty}|\partial \mathcal{E}|\left(u_{n^{\prime}}\right) \leq \Lambda+\varepsilon
$$

Due to (5.1c), $\left\{\xi_{n^{\prime}}\right\}$ is bounded in $B^{\prime}$, hence, up to a subsequence, we deduce that $\xi_{n^{\prime}} \rightarrow^{*} \xi$, with $\xi \in \partial \mathcal{E}(u)$ by (5.12). Thus, thanks to (5.14) and the weak*-lower semicontinuity of $\eta_{*}$ we deduce

$$
|\partial \mathcal{E}|(u) \leq \eta_{*}(-\xi) \leq \lim _{n^{\prime} \uparrow \infty}|\partial \mathcal{E}|\left(u_{n^{\prime}}\right) \leq \liminf _{n \uparrow \infty}|\partial \mathcal{E}|\left(u_{n}\right)+\varepsilon
$$

Being $\varepsilon$ arbitrary, (5.15) follows.

\section{Admissible functionals}

We are now in the position of introducing the broadest class of (energy) functionals which we are going to tackle in the framework of our metric approach to doubly nonlinear evolution equations.

Definition 5.4 (Admissible functionals). We say that a proper and lower semicontinuous functional $\mathcal{E}: B \rightarrow(-\infty,+\infty]$ is admissible if it can be decomposed into the $\operatorname{sum} \mathcal{E}=\mathcal{E}_{1}+\mathcal{E}_{2}$, the functionals $\mathcal{E}_{1}$ and $\mathcal{E}_{2}$ satisfying the following conditions

$\mathcal{E}_{1}$ is proper, $\lambda$-convex for some $\lambda \in \mathbb{R}$, lower semicontinuous, and bounded from below,

$\mathcal{E}_{2}$ is proper and

$$
\begin{aligned}
& \forall\left\{u_{n}\right\} \subset B,\left(u_{n} \rightarrow u, \sup _{n} \mathcal{E}_{1}\left(u_{n}\right)<+\infty\right) \Rightarrow \liminf _{n \uparrow \infty} \mathcal{E}_{2}\left(u_{n}\right) \geq \mathcal{E}_{2}(u) ; \\
& \forall M>0 \quad \exists 0<K_{1}<1, K_{2}>0 \text { s.t. } \\
& \mathcal{E}_{2}(u) \geq-K_{1} \mathcal{E}_{1}(u)-K_{2} \quad \forall u \in B \text { with }\|u\| \leq M, \\
& \forall u \in \operatorname{dom}(\mathcal{E}) \exists ! \xi=: \widetilde{D} \mathcal{E}_{2}(u) \in B^{\prime} \text { s.t. } \quad \forall\left\{u_{n}\right\} \subset B \text { with } \\
& u_{n} \rightarrow u, \sup _{n} \mathcal{E}_{1}\left(u_{n}\right)<+\infty \\
& \lim _{n \uparrow \infty} \frac{\mathcal{E}_{2}\left(u_{n}\right)-\mathcal{E}_{2}(u)-\left\langle\xi, u_{n}-u\right\rangle}{\left\|u_{n}-u\right\|}=0,
\end{aligned}
$$

$\forall M>0 \exists K_{3}>0$ s.t. $\left\|\widetilde{\mathrm{D}} \mathcal{E}_{2}(u)\right\|_{*} \leq K_{3} \forall u \in B$ with $\max \left(\|u\|, \mathcal{E}_{1}(u)\right) \leq M$.

$$
\forall\left\{u_{n}\right\} \subset B, u_{n} \rightarrow u, \sup _{n} \mathcal{E}_{1}\left(u_{n}\right)<+\infty \Rightarrow \widetilde{\mathrm{D}} \mathcal{E}_{2}\left(u_{n}\right) \rightarrow{ }^{*} \widetilde{\mathrm{D}} \mathcal{E}_{2}(u)
$$


Remark 5.5. Let us point out that with (5.18) and (5.20) we require $\mathcal{E}_{2}$ to be lower semicontinuous and (Fréchet) differentiable along sequences with bounded $\mathcal{E}_{1}$-energy and, further, (5.21) states that $\widetilde{\mathrm{D}} \mathcal{E}_{2}$ is estimated by the functional $\mathcal{E}_{1}$ and (5.22) that $\widetilde{\mathrm{D}} \mathcal{E}_{2}$ is continuous, again along sequences with bounded $\mathcal{E}_{1}$-energy. In other words, the functional $\mathcal{E}_{2}$ is a dominated perturbation of the $(\lambda)$-convex functional $\mathcal{E}_{1}$. In [45] a similar class of dominated concave perturbations of convex functionals was considered.

The following result collects some properties of the Fréchet subdifferential and of the slopes of admissible functionals, extending Lemma 5.3.

Proposition 5.6. Let $\mathcal{E}: B \rightarrow(-\infty,+\infty]$ be an admissible functional (with $\mathcal{E}=$ $\mathcal{E}_{1}+\mathcal{E}_{2}$ in the sense of Definition 5.4), and let $\eta$ be a positively homogeneous functional fulfilling $(5.1 \mathrm{a}, \mathrm{b}, \mathrm{c})$ and inducing the asymmetric distance $\Delta(5.2)$. Then,

1. for all $u \in \operatorname{dom}(\mathcal{E})$ the Fréchet subdifferential $\partial \mathcal{E}(u)$ is a convex and weakly* closed set,

$$
\partial \mathcal{E}(u)=\partial \mathcal{E}_{1}(u)+\widetilde{\mathrm{D}} \mathcal{E}_{2}(u) \quad \forall u \in \operatorname{dom}(\partial \mathcal{E}),
$$

and $\partial \mathcal{E}$ satisfies the strong-weak* closedness property along sequences with bounded energy

$$
u_{n} \rightarrow u, \quad \xi_{n} \rightarrow^{*} \xi, \quad \xi_{n} \in \partial \mathcal{E}\left(u_{n}\right) \quad \sup _{n}\left|\mathcal{E}\left(u_{n}\right)\right|<+\infty \Rightarrow \xi \in \partial \mathcal{E}(u)
$$

2. for all $u \in \operatorname{dom}(\mathcal{E})$

$$
\begin{gathered}
\partial \mathcal{E}(u) \neq \emptyset \Leftrightarrow|\partial \mathcal{E}|(u)<+\infty \quad \text { and } \quad|\partial \mathcal{E}|(u)=\min _{\xi \in \partial \mathcal{E}(u)} \eta_{*}(-\xi), \\
\left|\partial^{-} \mathcal{E}\right|(u)=|\partial \mathcal{E}|(u) \quad \forall u \in \operatorname{dom}(\partial \mathcal{E}) .
\end{gathered}
$$

Proof. First of all, we show that

$$
\partial \mathcal{E}(u)-\widetilde{\mathrm{D}} \mathcal{E}_{2}(u) \subset \partial \mathcal{E}_{1}(u) \quad \forall u \in \operatorname{dom}(\partial \mathcal{E}) .
$$

Indeed, let us fix any $\zeta \in \partial \mathcal{E}(u)$ : since $\mathcal{E}_{1}$ is convex, in order to show that $\zeta-$ $\widetilde{\mathrm{D}} \mathcal{E}_{2}(u) \in \partial \mathcal{E}_{1}(u)$ it is sufficient to check that for all sequence $\left\{w_{n}\right\}$ with $w_{n} \rightarrow u$ we have

$$
\begin{aligned}
& \liminf _{n \uparrow \infty} \frac{\mathcal{E}_{1}\left(w_{n}\right)-\mathcal{E}_{1}(u)-\left\langle\xi-\widetilde{\mathrm{D}} \mathcal{E}_{2}(u), w_{n}-u\right\rangle}{\left\|w_{n}-u\right\|} \\
& =\liminf _{n \uparrow \infty}\left(\frac{\mathcal{E}\left(w_{n}\right)-\mathcal{E}(u)-\left\langle\xi, w_{n}-u\right\rangle}{\left\|w_{n}-u\right\|}-\frac{\mathcal{E}_{2}\left(w_{n}\right)-\mathcal{E}_{2}(u)-\left\langle\widetilde{\mathrm{D}}_{2}(u), w_{n}-u\right\rangle}{\left\|w_{n}-u\right\|}\right) \\
& \geq 0 .
\end{aligned}
$$


Now, we may suppose that $\sup _{n} \mathcal{E}_{1}\left(w_{n}\right)<+\infty$, hence (5.26) trivially ensues from the fact that $\zeta \in \partial \mathcal{E}(u)$ and from the definition of $\widetilde{\mathrm{D}} \mathcal{E}_{2}(u)$. The proof of the converse inclusion $\partial \mathcal{E}_{1}(u)+\widetilde{\mathrm{D}} \mathcal{E}_{2}(u) \subset \partial \mathcal{E}(u)$ for all $u \in \operatorname{dom}(\partial \mathcal{E})$ follows the same lines. Thanks to $(5.23)$ and to Lemma 5.3, we immediately have that $\partial \mathcal{E}(u)$ is a convex and weakly* closed subset of $B^{\prime}$. Further, (5.24) is a consequence of (5.12) and of (5.22).

In order to show the first of (5.25) at a point $u \in \operatorname{dom}(\mathcal{E})$, we may suppose without loss of generality that $\widetilde{\mathrm{D}} \mathcal{E}_{2}(u)=0$ and that $|\partial \mathcal{E}|(u)>0$. Then, using (5.19) one easily checks that there exists some constant $C>0$ such that

$$
|\partial \mathcal{E}|(u)=\limsup _{v \rightarrow u, \mathcal{E}_{1}(v) \leq C} \frac{\left(\mathcal{E}_{1}(u)-\mathcal{E}_{1}(v)+\mathcal{E}_{2}(u)-\mathcal{E}_{2}(v)\right)^{+}}{\eta(v-u)} .
$$

Observing that

$$
\lim _{v \rightarrow u, \mathcal{E}_{1}(v) \leq C} \frac{\left(\mathcal{E}_{2}(u)-\mathcal{E}_{2}(v)\right)^{+}}{\eta(v-u)}=0,
$$

we conclude

$$
|\partial \mathcal{E}|(u)=\limsup _{v \rightarrow u, \mathcal{E}_{1}(v) \leq C} \frac{\left(\mathcal{E}_{1}(u)-\mathcal{E}_{1}(v)\right)^{+}}{\eta(v-u)}=\min _{\xi \in \partial \mathcal{E}_{1}(u)} \eta_{*}(-\xi)=\min _{\xi \in \partial \mathcal{E}(u)} \eta_{*}(-\xi),
$$

the second identity due to (5.14) for the $\lambda$-convex functional $\mathcal{E}_{1}$ and the third one to (5.23). As for the second of (5.25), one clearly has $\left|\partial^{-} \mathcal{E}\right|(u) \leq|\partial \mathcal{E}|(u)$; in order to prove the converse inequality, we argue in the same way as for proving (5.15).

Remark 5.7. Combining (5.23) with the representation (5.25) of the slope of functionals $\mathcal{E}$ and $\mathcal{E}_{1}$, one deduces that

$$
\begin{aligned}
\left|\partial \mathcal{E}_{1}\right|(u)=\min _{\xi \in \partial \mathcal{E}(u)-\widetilde{\mathrm{D}} \mathcal{E}_{2}(u)} \eta_{*}(-\xi) & \leq \min _{\xi \in \partial \mathcal{E}(u)} \eta_{*}(-\xi)+\eta_{*}\left(\widetilde{\mathrm{D}} \mathcal{E}_{2}(u)\right) \\
& \leq|\partial \mathcal{E}|(u)+K\left\|\widetilde{\mathrm{D}} \mathcal{E}_{2}(u)\right\|_{*} \quad \forall u \in B,
\end{aligned}
$$

where the first inequality follows from the sublinearity of $\eta_{*}$ and the second one from (5.5).

For later convenience, we also state a version of the mean-value theorem for the functional $\mathcal{E}_{2}$ which can be proved exactly in the same way as [2, Chapter 1, Theorem 1.8], to which we refer the reader.

Lemma 5.8. Let $\mathcal{E}: B \rightarrow(-\infty,+\infty]$ be an admissible functional. Then, for all $u, v \in \operatorname{dom}(\mathcal{E})$ such that the segment $[u, v] \subset \operatorname{dom}(\mathcal{E})$ one has

$$
\left|\mathcal{E}_{2}(u)-\mathcal{E}_{2}(v)\right| \leq \sup _{z \in[u, v]}\left\|\widetilde{\mathrm{D}} \mathcal{E}_{2}(z)\right\|_{*}\|u-v\| .
$$

We conclude the section with a technical result, which will turn out to be useful in the sequel. 
Lemma 5.9 (Selection of optimal directions). Let us suppose that

$$
B \text { is reflexive, }
$$

let $\mathcal{E}$ be an admissible functional and let $\xi \in \partial \mathcal{E}(u)$ with $0<\eta_{*}(-\xi)=|\partial \mathcal{E}|(u)<$ $+\infty$. Then, there exist a sequence of directions $\left\{v_{n}\right\}, v \in B$ and a positive vanishing sequence $h_{n} \in(0,+\infty)$ such that

$$
\eta\left(v_{n}\right)=\eta(v)=1, \quad\langle-\xi, v\rangle=\eta_{*}(-\xi), \quad v_{n} \rightarrow v
$$

and the sequence $u_{n}:=u+h_{n} v_{n}$ satisfies

$$
\lim _{n \uparrow+\infty} \frac{\mathcal{E}(u)-\mathcal{E}\left(u_{n}\right)}{\eta\left(u_{n}-u\right)}=\lim _{n \uparrow+\infty} \frac{\mathcal{E}(u)-\mathcal{E}\left(u+h_{n} v_{n}\right)}{h_{n}}=|\partial \mathcal{E}|(u)=\eta_{*}(-\xi) .
$$

Proof. By the definition of slope and by (5.25), we can find a sequence $u_{n} \neq u$ strongly converging to $u$ such that

$$
\lim _{n \uparrow+\infty} \frac{\mathcal{E}(u)-\mathcal{E}\left(u_{n}\right)}{\eta\left(u_{n}-u\right)}=|\partial \mathcal{E}|(u)=\eta_{*}(-\xi)>0 .
$$

We thus set

$$
h_{n}:=\eta\left(u_{n}-u\right), \quad v_{n}:=\frac{u_{n}-u}{h_{n}},
$$

and, owing to the reflexivity of $B$, we can extract a subsequence (still labeled $v_{n}$ ) weakly converging to $v \in B$ with $\eta(v) \leq 1$. By the subdifferentiability assumption, we have

$$
\mathcal{E}\left(u_{n}\right)-\mathcal{E}(u) \geq\left\langle\xi, u_{n}-u\right\rangle+o\left(h_{n}\right) \text { as } n \uparrow+\infty ;
$$

dividing by $h_{n}$ and inverting the direction of the inequality, we can pass to the limit as $n \uparrow+\infty$, obtaining

$$
\eta_{*}(-\xi) \leq\langle-\xi, v\rangle \leq \eta_{*}(-\xi) \cdot \eta(v),
$$

thus proving $\eta(v)=1$ and the second identity of (5.30).

\subsection{Chain rule for admissible families of time-dependent functionals}

In this section, we shall establish a general chain-rule formula for a family of time-dependent functionals $\mathcal{E}_{t}(\cdot), t \in[0, T]$. The natural assumptions combine the admissibility conditions given in Definition 5.4, the conditions on the timedependence discussed in Section 2.3, and some of the topological assumptions of Section 2.5. We recall them in a unique definition. 
Definition 5.10 (Admissible family of time-dependent functionals). We say that a family of (proper, lower semicontinuous) functionals $\mathcal{E}_{t}: B \rightarrow(-\infty,+\infty]$, $t \in[0, T]$, is admissible if each functional $\mathcal{E}_{t}$ is admissible according to Definition 5.4, with the decomposition $\mathcal{E}_{t}=\mathcal{E}_{t}^{1}+\mathcal{E}_{t}^{2}$ for all $t \in[0, T]$, and

1. $\operatorname{dom}\left(\mathcal{E}_{t}\right) \equiv \mathrm{D}$ does not depend on time,

2. the functionals $\mathcal{E}_{t}^{1}$ are uniformly bounded from below with respect to $t$ and $\lambda$ uniformly convex, namely

$$
\begin{aligned}
& \exists \lambda \in \mathbb{R}: \quad \forall t \in[0, T] \forall u_{0}, u_{1} \in B \forall \theta \in[0,1] \\
& \mathcal{E}_{t}^{1}\left((1-\theta) u_{0}+\theta u_{0}\right) \leq(1-\theta) \mathcal{E}_{t}^{1}\left(u_{0}\right)+\theta \mathcal{E}_{t}^{1}\left(u_{1}\right)-\frac{1}{2} \lambda \theta(1-\theta)\left\|u_{0}-u_{1}\right\|^{2},
\end{aligned}
$$

3. conditions (5.19) and (5.21) hold with constants $K_{1}, K_{2}$, and $K_{3}$ independent of $t$,

4. for every sequences $v_{n}, v \in \mathrm{D}, t_{n}, t \in[0, T]$

$$
v_{n} \rightarrow v, t_{n} \rightarrow t, \sup _{n} \mathcal{E}_{t_{n}}(v)<+\infty \Rightarrow \exists \lim _{n \rightarrow \infty} \frac{\mathcal{E}_{t_{n}}\left(v_{n}\right)-\mathcal{E}_{t}\left(v_{n}\right)}{t_{n}-t}=: \partial_{t} \mathcal{E}_{t}(v),
$$

which satisfies

$$
\left|\partial_{t} \mathcal{E}_{t}(v)\right| \leq K_{4}\left(\mathcal{E}_{t}(v)+\|v\|+1\right) \quad \forall t \in[0, T], v \in \mathrm{D}
$$

for a suitable constant $K_{4} \geq 0$.

In the following formula we choose a positively homogeneous and convex functional $\eta$ satisfying $(5.1 \mathrm{a}, \mathrm{b}, \mathrm{c})$ and, given an absolutely continuous curve $v$, we denote by $\left|v^{\prime}\right|(t)$ its metric derivative with respect to the asymmetric distance $\Delta(5.2)$ induced by $\eta$.

Proposition 5.11 (Chain rule). Let $\mathcal{E}:[0, T] \times B \rightarrow(-\infty,+\infty]$ be an admissible family of functionals according to Definition 5.10, let $\eta$ be a positively homogeneous functional fulfilling (5.1a,b,c) and inducing the asymmetric distance $\Delta(5.2)$, and let $v \in \mathrm{AC}(0, T ; B)$ be an absolutely continuous curve satisfying

$$
\sup _{t \in[0, T]} \mathcal{E}_{t}(v(t))<+\infty, \quad \int_{0}^{T}\left|v^{\prime}\right|(t) \cdot\left|\partial \mathcal{E}_{t}\right|(v(t)) \mathrm{d} t<+\infty .
$$

Then, the map $t \mapsto \mathcal{E}_{t}(v(t))$ is absolutely continuous and

$$
\frac{d}{d t} \mathcal{E}_{t}(v(t)) \geq \partial_{t} \mathcal{E}_{t}(v(t))-\left|\partial \mathcal{E}_{t}\right|(v(t)) \cdot\left|v^{\prime}\right|(t) \quad \text { for a.e. } t \in(0, T) .
$$

Moreover, if $v$ is (weakly) differentiable a.e., we have

$$
\frac{d}{d t} \mathcal{E}_{t}(v(t))=\partial_{t} \mathcal{E}_{t}(v(t))+\left\langle\xi, v^{\prime}(t)\right\rangle \quad \forall \xi \in \partial \mathcal{E}_{t}(v(t)) \quad \text { for a.e. } t \in(0, T) \text {. }
$$


Proof. Up to a suitable reparametrization (see [5, Lemma 1.1.4]), it is possible to assume that the curve is 1-Lipschitz (with respect to the norm of $B$ ) and

$$
\left|v^{\prime}\right|(t) \leq K \quad \text { for a.e. } t \in(0, T) .
$$

Since $v$ is uniformly bounded and the energies $\mathcal{E}_{t}(v(t))$ are uniformly bounded, using (5.19) we get

$$
\sup _{t \in[0, T]} \mathcal{E}_{t}^{1}(v(t))<+\infty
$$

as well as

$$
\mathcal{F}(v(s))+\left|\partial_{t} \mathcal{F}_{t}(v(s))\right| \leq S<+\infty \quad \forall s, t \in[0, T],
$$

where $\mathcal{F}$ is defined as in (2.21). In order to show the absolute continuity of the energy map $t \mapsto \mathcal{E}_{t}(v(t))$, we need to estimate

$$
\begin{gathered}
\mathcal{E}_{t}(v(t))-\mathcal{E}_{s}(v(s))=\left(\mathcal{E}_{t}(v(t))-\mathcal{E}_{t}(v(s))\right)+\left(\mathcal{E}_{t}(v(s))-\mathcal{E}_{s}(v(s))\right) \\
\text { for } 0 \leq s<t \leq T .
\end{gathered}
$$

Thanks to (5.40), we have

$$
\mathcal{E}_{t}(v(s))-\mathcal{E}_{s}(v(s)) \leq S|t-s| .
$$

In order to estimate the first summand on the right-hand side of (5.41), we notice that, thanks to (5.39), the convexity of $\mathcal{E}_{t}^{1}$, and (5.21),

$$
\sup _{z \in[v(s), v(t)]} \mathcal{E}_{t}^{1}(z)<+\infty, \text { whence } \sup _{z \in[v(s), v(t)]}\left\|\widetilde{\mathrm{D}} \mathcal{E}_{t}^{2}(z)\right\|_{*} \leq S_{1}<+\infty,
$$

for a positive constant $S_{1}$. Hence, Lemma 5.8 and the 1-Lipschitz continuity of $v$ yield

$$
\begin{gathered}
\mathcal{E}_{t}^{2}(v(t))-\mathcal{E}_{t}^{2}(v(s)) \leq \sup _{z \in[v(s), v(t)]}\left\|\widetilde{\mathrm{D}} \mathcal{E}_{t}^{2}(z)\right\|_{*}\|v(t)-v(s)\| \leq S_{1}|t-s| \\
\text { for } 0 \leq s<t \leq T .
\end{gathered}
$$

On the other hand, thanks to (5.13)

$$
\begin{aligned}
\mathcal{E}_{t}^{1}(v(t))-\mathcal{E}_{t}^{1}(v(s)) & \leq\left|\partial \mathcal{E}_{t}^{1}\right|(v(t)) \eta(v(s)-v(t))+\frac{1}{2} \lambda^{-} K^{2} \eta^{2}(v(s)-v(t)) \\
& \leq\left(K\left|\partial \mathcal{E}_{t}\right|(v(t))+K^{2} S_{1}+\frac{1}{2} \lambda^{-} K^{4}\right)|t-s|,
\end{aligned}
$$

the latter inequality due to (5.27), (5.38) and again (5.43). Combining (5.41), (5.42), (5.44), and (5.45), and inverting the role of $s$ and $t$ we easily get the following estimate

$$
\left|\mathcal{E}_{t}(v(t))-\mathcal{E}_{s}(v(s))\right| \leq\left(K\left|\partial \mathcal{E}_{t}\right|(v(t))+K\left|\partial \mathcal{E}_{s}\right|(v(s))+C\right)|t-s|
$$


for some suitable constant $C$. Arguing as in [5, Theorem 1.2.5], we get the absolute continuity of the energy.

Let us now fix a point $s \in(0, T)$ such that $\left|v^{\prime}\right|(s)$ and $\frac{d}{d t} \mathcal{E}_{S}(v(s))$ exist, and $\left|\partial \mathcal{E}_{s}\right|(v(s))<+\infty$. Equality (5.41) and the definition of slope yield when $t \rightarrow s$

$\mathcal{E}_{t}(v(t))-\mathcal{E}_{s}(v(s)) \geq-\left|\partial \mathcal{E}_{S}\right|(v(s)) \eta(v(t)-v(s))+\partial_{t} \mathcal{E}_{S}\left(v_{s}\right)(t-s)+o(|t-s|)$

so that, dividing the inequality by $t-s>0$, we get (5.36). When $v$ is also weakly differentiable at $s$, we can use the definition of Fréchet subdifferential to obtain

$$
\begin{aligned}
\mathcal{E}_{t}(v(t))-\mathcal{E}_{s}(v(s)) \geq & \langle\xi, v(t)-v(s)\rangle+\partial_{t} \mathcal{E}_{s}\left(v_{s}\right)(t-s)+o(|t-s|) \\
& \forall \xi \in \partial \mathcal{E}_{s}(v(s)) .
\end{aligned}
$$

Dividing by $t-s$ and passing to the limit first as $t \downarrow s$ and then as $t \uparrow s$, we conclude.

\section{Finsler metrics}

In this section, we want to extend some of the previous results to the case in which $\Delta$ is an nondegenerate asymmetric Finsler distance induced by a family of convex and positively homogeneous (sublinear) functionals $\eta_{u}$, depending on $u \in B$ (and again we take as $\sigma$ the norm topology of $B$ ).

We consider the case in which

$$
B \text { is a separable and reflexive Banach space, }
$$

endowed with a family of functionals

$$
\begin{aligned}
& \eta_{u}: B \rightarrow[0,+\infty), u \in B, \\
& \text { satisfying conditions }(5.1 \mathrm{a}, \mathrm{b}, \mathrm{c}) \text { with } K \text { independent of } u .
\end{aligned}
$$

We are assuming that the dependence of $\eta$ with respect to $u$ is continuous in the sense of Mosco (see, e.g., [9, Section 3.3, page 295]), i.e., whenever a sequence $u_{n}$ is strongly convergent to $u$ in $B$ as $n \uparrow \infty$, the corresponding sequence of functionals $\eta_{u_{n}}$ Mosco-converges to $\eta_{u}$. This means two conditions:

$$
u_{n} \rightarrow u, \quad v_{n} \rightarrow v \quad \text { in } B \quad \Rightarrow \quad \liminf _{n \rightarrow \infty} \eta_{u_{n}}\left(v_{n}\right) \geq \eta_{u}(v),
$$

and

$$
u_{n} \rightarrow u, \quad v \in B \quad \Rightarrow \quad \exists v_{n} \rightarrow v: \quad \lim _{n \rightarrow \infty} \eta_{u_{n}}\left(v_{n}\right)=\eta_{u}(v)
$$

Let us recall a well-known consequence of this assumption: 
Theorem 6.1 (Duality for Mosco-convergence). If $u_{n} \rightarrow u$ in $B$ and $\xi_{n} \rightarrow^{*} \xi$ in $B^{\prime}$, then

$$
\liminf _{n \rightarrow \infty} \eta_{u_{n} *}\left(\xi_{n}\right) \geq \eta_{u *}(\xi) .
$$

Proof. We fix $v \in B$ with $\eta_{u}(v)=1$ and we take a sequence $v_{n}$ satisfying (6.4). We have

$$
\liminf _{n \uparrow+\infty} \eta_{u_{n} *}\left(\xi_{n}\right) \geq \liminf _{n \uparrow+\infty} \frac{\left\langle\xi_{n}, v_{n}\right\rangle}{\eta_{u_{n}}\left(v_{n}\right)}=\langle\xi, v\rangle .
$$

Since $v$ can be arbitrarily chosen with $\eta_{u}(v)=1$, taking the supremum of the last duality with respect to $v$ we conclude.

\section{The induced asymmetric Finsler distance}

We introduce $\Delta$ through the formula

$$
\begin{aligned}
\Delta(v, w):= & \inf \left\{\int_{0}^{1} \eta_{u(t)}\left(u^{\prime}(t)\right) \mathrm{d} t: u \in \mathrm{AC}(0,1 ; B), u(0)=v, u(1)=w\right\} \\
& \forall v, w \in B .
\end{aligned}
$$

Note that (6.6) makes sense (the map $t \mapsto \eta_{u(t)}\left(u^{\prime}(t)\right)$ is integrable for all $u \in$ $\mathrm{AC}(0,1 ; B))$ and defines a (possibly non-symmetric) distance (in the sense of (2.2)) on $B$, associated with the family $\left\{\eta_{u}\right\}_{u \in B}$. Since (5.1c) holds uniformly with respect to $u \in B$, we have

$$
K^{-1}\|v-w\| \leq \Delta(v, w) \leq K\|v-w\| \quad \forall v, w \in B .
$$

Therefore, the class of absolutely continuous curves with respect to $\Delta$ coincides with the usual one (i.e. with respect to the norm of $B$ ). Again, we shall use the notation (5.3) for slopes with respect to $\Delta$.

The main problem is to characterize the metric velocity associated with $\Delta$; here is the main result:

Theorem 6.2 (Metric velocity). Assume (6.1)-(6.4), and let $\Delta$ be the asymmetric distance (6.6); let $u \in \mathrm{AC}(a, b ; B)$ and let $\left|u^{\prime}\right|$ be its (a.e. defined) metric velocity, induced by $\Delta$. We have

$$
\left|u^{\prime}\right|(t)=\eta_{u(t)}\left(u^{\prime}(t)\right) \quad \text { for a.e. } t \in(a, b) .
$$

More precisely, the identity in (6.8) holds at each point $\bar{t}$ fulfilling the following three conditions:

i) $u$ is differentiable at $\bar{t}$,

ii) $\bar{t}$ is a Lebesgue point for the map $t \mapsto \eta_{u(t)}\left(u^{\prime}(t)\right)$,

iii) $\exists \lim _{h \downarrow 0} \frac{\Delta(u(\bar{t}), u(\bar{t}+h))}{h}=:\left|u^{\prime}\right|(\bar{t})$. 
We split the proof in various steps.

Lemma 6.3. Under the same assumptions as in Theorem 6.2, let $\bar{t}$ be satisfying conditions i)... iii). We have

$$
\left|u^{\prime}\right|(\bar{t}) \leq \eta_{u(\bar{t})}\left(u^{\prime}(\bar{t})\right) .
$$

Proof. For $h>0$ let us consider the curve $r \in[0,1] \mapsto \gamma(r):=u(\bar{t}+r h)$ connecting $u(\bar{t})$ and $u(\bar{t}+h)$. By definition of $\Delta$ and a trivial change of variables, we obtain

$$
\Delta(u(\bar{t}), u(\bar{t}+h)) \leq \int_{\bar{t}}^{\bar{t}+h} \eta_{u(r)}\left(u^{\prime}(r)\right) \mathrm{d} r .
$$

Dividing by $h>0$ we get (6.9), being $\bar{t}$ a Lebesgue point of the map $r \mapsto$ $\eta_{u(r)}\left(u^{\prime}(r)\right)$.

The next lemma provides the crucial technical result for the proof of Theorem 6.2, which will also be useful later on.

Lemma 6.4. Let $u, u_{n} \in B$ and $h_{n}>0$ such that as $n \uparrow+\infty$

$$
u_{n} \rightarrow u, \quad \frac{u_{n}-u}{h_{n}} \rightarrow v \neq 0, \quad \frac{\eta_{u}\left(u_{n}-u\right)}{h_{n}} \rightarrow \eta_{u}(v) .
$$

Then,

$$
\liminf _{n \uparrow+\infty} \frac{\Delta\left(u, u_{n}\right)}{h_{n}} \geq \eta_{u}(v), \quad \liminf _{n \uparrow+\infty} \frac{\Delta\left(u, u_{n}\right)}{\eta_{u}\left(u_{n}-u\right)} \geq 1 .
$$

Proof. By the definition (6.6) of $\Delta$ and a standard reparametrization argument, we find Lipschitz continuous curves $\gamma_{n}:[0,1] \rightarrow B$ connecting $u$ to $u_{n}$ and a vanishing positive sequence $\varepsilon_{n} \in(0,1 / 2)$ such that

$$
\Delta\left(u, u_{n}\right) \geq\left(1-\varepsilon_{n}\right) \int_{0}^{1} \eta_{\gamma_{n}(t)}\left(\gamma_{n}^{\prime}(t)\right) \mathrm{d} t, \quad\left\|\gamma_{n}^{\prime}(t)\right\| \leq 4 K \Delta\left(u, u_{n}\right)
$$

for a.e. $t \in(0, T)$.

Dividing by $h_{n}$, we can assume that $h_{n}^{-1} \Delta\left(u, u_{n}\right) \leq A<+\infty$ for some constant $A>0$; by introducing the curve

$$
\hat{\gamma}_{n}(t):=u+\frac{\gamma_{n}(t)-u}{h_{n}}, \quad \text { with } \quad \hat{\gamma}_{n}^{\prime}(t)=h_{n}^{-1} \gamma_{n}^{\prime}(t)
$$

we get

$$
\begin{gathered}
\frac{\Delta\left(u, u_{n}\right)}{h_{n}} \geq\left(1-\varepsilon_{n}\right) \int_{0}^{1} \eta_{\gamma_{n}(t)}\left(\hat{\gamma}_{n}^{\prime}(t)\right) \mathrm{d} t, \quad\left\|\hat{\gamma}_{n}^{\prime}(t)\right\| \leq 4 K \frac{\Delta\left(u, u_{n}\right)}{h_{n}} \leq 4 K A \\
\text { for a.e. } t \in(0, T),
\end{gathered}
$$


so that

$$
\liminf _{n \uparrow+\infty} \frac{\Delta\left(u, u_{n}\right)}{h_{n}} \geq \liminf _{n \uparrow+\infty} \int_{0}^{1} \eta_{\gamma_{n}(t)}\left(\hat{\gamma}_{n}^{\prime}(t)\right) \mathrm{d} t .
$$

After the extraction of a suitable subsequence (not relabeled), we can assume that the last lim inf is in fact a limit and, since $\hat{\gamma}_{n}^{\prime}$ is uniformly bounded, that $\hat{\gamma}_{n}^{\prime} \rightarrow z$ weakly in $L^{2}(0,1 ; B)$, which is still a reflexive and separable Banach space. Since $\gamma_{n}(t) \rightarrow u$ uniformly as $n \rightarrow \infty$, general lower semicontinuity results for normal integrands applied to the strongly-weakly lower semicontinuous functional $(u, v) \mapsto \eta_{u}(v)$ (see [11, Theorem 3.2], as well as [45, Theorem 3.2] and [35, Theorem B.1]) yield

$$
\liminf _{n \uparrow+\infty} \int_{0}^{1} \eta_{\gamma_{n}(t)}\left(\hat{\gamma}_{n}^{\prime}(t)\right) \mathrm{d} t \geq \int_{0}^{1} \eta_{u}(z(t)) \mathrm{d} t \geq \eta_{u}(Z), \quad Z:=\int_{0}^{1} z(t) \mathrm{d} t,
$$

where the last passage follows by the convexity of $\eta_{u}$ and Jensen inequality.

On the other hand, we have

$$
\int_{0}^{1} \hat{\gamma}_{n}^{\prime}(t) \mathrm{d} t=\hat{\gamma}_{n}(1)-\hat{\gamma}_{n}(0)=\frac{u_{n}-u}{h_{n}},
$$

and therefore for every $y \in B^{\prime}$

$$
\begin{aligned}
\langle y, Z\rangle & =\int_{0}^{1}\langle y, z(t)\rangle \mathrm{d} t \\
& =\lim _{n \rightarrow \infty} \int_{0}^{1}\left\langle y, \hat{\gamma}_{n}^{\prime}(t)\right\rangle \mathrm{d} t=\lim _{n \rightarrow \infty}\left\langle y, h_{n}^{-1}\left(u_{n}-u\right)\right\rangle=\langle y, v\rangle,
\end{aligned}
$$

which yields $Z=v$ and, by (6.13),

$$
\liminf _{n \uparrow+\infty} \frac{\Delta\left(u, u_{n}\right)}{h_{n}} \geq \eta_{u}(v) .
$$

We conclude that

$$
\liminf _{n \uparrow+\infty} \frac{\Delta\left(u, u_{n}\right)}{\eta_{u}\left(u_{n}-u\right)}=\liminf _{n \uparrow+\infty} \frac{\Delta\left(u, u_{n}\right)}{h_{n}} \cdot \frac{h_{n}}{\eta_{u}\left(u_{n}-u\right)} \geq \eta_{u}(v) \frac{1}{\eta_{u}(v)}=1 .
$$

Proof of Theorem 6.2. We can conclude now the proof of Theorem 6.2 by proving the opposite inequality

$$
\left|u^{\prime}\right|(\bar{t}) \geq \eta_{u(\bar{t})}\left(u^{\prime}(\bar{t})\right)
$$

at each point $\bar{t}$ satisfying conditions i)-iii).

It is obviously not restrictive to assume $u^{\prime}(\bar{t}) \neq 0$ : we can thus apply the previous Lemma, choosing a positive vanishing sequence $h_{n} \downarrow 0$ and $u:=u(\bar{t}), u_{n}:=$ $u\left(\bar{t}+h_{n}\right), v=u^{\prime}(\bar{t})$. 
We apply now Lemma 6.4 to prove a useful property of the $\Delta$-slope of an admissible functional.

Theorem 6.5. Let $\mathcal{E}$ be an admissible functional in the sense of Definition 5.4, let $|\partial \mathcal{E}|$ be the slope associated with the asymmetric distance (6.6), and let $\partial \mathcal{E}$ be its Fréchet subdifferential. Then, for every $u \in \operatorname{dom}(\mathcal{E})$

$$
\begin{gathered}
|\partial \mathcal{E}|(u)<+\infty \quad \Leftrightarrow \quad \partial \mathcal{E}(u) \neq \emptyset \\
|\partial \mathcal{E}|(u) \leq K\|\xi\| \quad \forall \xi \in \partial \mathcal{E}(u),
\end{gathered}
$$

and, in this case,

$$
|\partial \mathcal{E}|(u) \geq \min _{\xi \in \partial \mathcal{E}(u)} \eta_{u *}(-\xi) .
$$

Proof. Since the asymmetric distance $\Delta$ satisfies the uniform bound (6.7), it is immediate to check that the $\Delta$-slope of a functional $\mathcal{E}$ is finite if and only if the slope of $\mathcal{E}$ with respect to the norm of $B$ is finite: (5.25) thus yields (6.19), the second estimate following from (6.2).

In order to check (6.20), we fix $u \in \operatorname{dom}(\partial \mathcal{E})$, we choose an element $\xi_{0} \in$ $\partial \mathcal{E}(u)$ which attains the minimum in (6.20) (it is not restrictive to assume $\xi_{0} \neq 0$ ) and we apply Lemma 5.9: we then find a sequence $u_{n} \in \operatorname{dom}(\mathcal{E})$ such that

$$
\begin{aligned}
& h_{n}:=\eta_{u}\left(u_{n}-u\right) \rightarrow 0, \quad \frac{u_{n}-u}{h_{n}} \rightarrow v, \quad \eta_{u}(v)=1, \\
& \lim _{n \uparrow+\infty} \frac{\mathcal{E}(u)-\mathcal{E}\left(u_{n}\right)}{h_{n}}=\eta_{u *}\left(-\xi_{0}\right)>0 .
\end{aligned}
$$

On the other hand, Lemma 6.4 yields

$$
\begin{aligned}
|\partial \mathcal{E}|(u) & \geq \limsup _{n \rightarrow \infty} \frac{\mathcal{E}(u)-\mathcal{E}\left(u_{n}\right)}{\Delta\left(u, u_{n}\right)}=\lim _{n \uparrow+\infty} \frac{\mathcal{E}(u)-\mathcal{E}\left(u_{n}\right)}{h_{n}} \cdot \limsup _{n \uparrow+\infty} \frac{h_{n}}{\Delta\left(u, u_{n}\right)} \\
& \geq \eta_{u *}\left(-\xi_{0}\right) \cdot \eta_{u}(v)=\eta_{u *}\left(-\xi_{0}\right) .
\end{aligned}
$$

Taking into account Theorem 6.1 and Proposition 5.6, we easily get

Corollary 6.6. Let $\mathcal{E}$ be an admissible functional and let $\left|\partial^{-} \mathcal{E}\right|$ be the relaxed slope associated with the asymmetric distance (6.6), i.e.

$$
\left|\partial^{-} \mathcal{E}\right|(u):=\inf \left\{\liminf _{n \uparrow \infty}|\partial \mathcal{E}|\left(u_{n}\right): u_{n} \rightarrow u, \sup _{n} \mathcal{E}\left(u_{n}\right)<+\infty\right\} .
$$

Then, for every $u \in \operatorname{dom}(\mathcal{E})$

$$
\left|\partial^{-} \mathcal{E}\right|(u)<+\infty \quad \Leftrightarrow \quad \partial \mathcal{E}(u) \neq \emptyset
$$

and, in this case,

$$
\left|\partial^{-} \mathcal{E}\right|(u) \geq \min _{\xi \in \partial \mathcal{E}(u)} \eta_{u *}(-\xi) .
$$


Theorem 6.7 (Relaxed slope and chain rule for admissible functionals). Let us consider an admissible family of functionals (according to Definition 5.10) $\mathcal{E}_{t}$ : $B \rightarrow(-\infty,+\infty], t \in[0, T]$, and let $\Delta$ be the asymmetric Finsler distance induced by (6.6) under the assumptions of Section 6. Then, the relaxed slope $\left|\partial^{-} \mathcal{E}_{t}\right|$ satisfies the chain rule condition of Definition 2.5: for any curve $v \in \operatorname{AC}(0, T ; B)$ with

$$
\int_{0}^{T}\left|v^{\prime}\right|(t) \cdot\left|\partial^{-} \mathcal{E}_{t}\right|(v(t)) \mathrm{d} t<+\infty, \quad \sup _{t \in(0, T)} \mathcal{E}_{t}(v(t))<+\infty,
$$

the map $t \mapsto \mathcal{E}_{t}(v(t))$ is absolutely continuous, and

$$
\frac{d}{d t} \mathcal{E}_{t}(v(t)) \geq \partial_{t} \mathcal{E}(t, v(t))-\left|v^{\prime}\right|(t) \cdot\left|\partial^{-} \mathcal{E}_{t}\right|(v(t)) \quad \text { for a.e. } t \in(0, T)
$$

Proof. Since the asymmetric distance $\Delta$ is metrically equivalent to the distance induced by the norm, also on account of Proposition 5.6 inequality (6.24) yields (5.35) and we may apply Proposition 5.11. Being $B$ reflexive, $v$ is differentiable a.e., and therefore (5.37) yields for a.e. $t \in(0, T)$ and every $\xi \in \partial \mathcal{E}_{t}(v(t))$

$$
\begin{aligned}
\frac{d}{d t} \mathcal{E}_{t}(v(t)) & =\partial_{t} \mathcal{E}_{t}(v(t))+\left\langle\xi, v^{\prime}(t)\right\rangle \\
& \geq \partial_{t} \mathcal{E}_{t}(v(t))-\eta_{v(t)}\left(v^{\prime}(t)\right) \cdot \min _{\xi \in \partial \mathcal{E}_{t}(v(t))} \eta_{v(t) *}(-\xi) \\
& \geq \partial_{t} \mathcal{E}_{t}(v(t))-\left|v^{\prime}\right|(t) \cdot\left|\partial^{-} \mathcal{E}_{t}\right|(v(t))
\end{aligned}
$$

the last inequality being a consequence of (6.8) and (6.23).

\section{Metric evolutions in $L^{1}(\Omega)$}

\section{Notation}

In this section and in the next one, we shall denote by $\Omega$ a bounded domain of $\mathbb{R}^{d}$, $d \geq 1$, by $\|\cdot\|_{r}$ the norm of the space $L^{r}(\Omega), 1 \leq r \leq \infty$, and by $\langle\cdot, \cdot\rangle$ the duality pairing between $H^{-1}(\Omega)$ and $H_{0}^{1}(\Omega)$.

\section{Setup}

Throughout this section, we shall drop the reflexivity assumption (6.1) and we shall focus on the prototypical case in which

the ambient Banach space $B$ is $L^{1}(\Omega)$, and $\eta$ is the norm functional $\|\cdot\|_{1}$.

As we already mentioned in the Introduction, $L^{1}(\Omega)$ does not enjoy the RadonNikodým property. A simple example of an absolutely continuous curve $u:[0, T] \rightarrow$ 
$L^{1}(\Omega)$ which is not a.e. differentiable can be constructed, in the case $\Omega=(0,1)$, in the following way: we take an absolutely continuous map $s:[0, T] \rightarrow[0,1]$ and a function $a \in L^{1}(0,1)$, and we let

$$
u(x, t):=\left\{\begin{array}{ll}
0 & \text { if } x \in[0, s(t)], \\
a(x) & \text { if } x \in(s(t), 1]
\end{array} \quad \forall(x, t) \in[0,1] \times[0, T] .\right.
$$

However, we may compute the metric derivative

$$
\left|u^{\prime}\right|_{1}(t):=\lim _{h \rightarrow 0} \frac{\|u(t+h)-u(t)\|_{1}}{h} \text { for a.e. } t \in(0, T),
$$

of the above curve, obtaining $\left|u^{\prime}\right|_{1}(t)=\left|a(s(t)) s^{\prime}(t)\right|$ for a.e. $t \in(0, T)$.

Throughout this section, we shall analyze the metric Problem 2.6 for a given lower semicontinuous functional $\mathcal{E}:[0, T] \times L^{1}(\Omega) \rightarrow(-\infty,+\infty]$ in the gradient flow case, namely with the quadratic dissipation functional (1.16).

We shall start with some simple examples of (time-independent) energy functionals $\mathcal{E}$, in which it is possible to calculate explicitly a solution of the (Cauchy problem for the) associated differential inclusion (DNE) (driven by the energy $\mathcal{E}$ and the dissipation $\Psi$ (1.19)). Indeed, we shall show that the constructed solution also complies with the metric formulation of Problem 2.6.

Next, in Section 7.2 we shall focus on the sole metric evolution of a more general class of energy functionals ( $c f$. (7.19)). Exploiting the preliminary results obtained in Sections 5.2-5.3, we shall deduce from Theorem 3.5 the existence of a solution to the associated metric formulation, see Theorem 7.5 later on.

\subsection{Examples}

Example 7.1. We consider $\Omega=(0,1)$ and the quadratic energy functional $\mathcal{E}(1.17)$. We recall that the associated differential inclusion is (1.20), which we supplement with the initial datum

$$
u_{0}(x):=1-x \quad \forall x \in[0,1] .
$$

We look for a solution of the Cauchy problem $(1.20,7.3)$ having the form

$$
u(x, t):=\left\{\begin{array}{ll}
u_{0}(\zeta(t)) & \text { if } x \in[0, \zeta(t)], \\
u_{0}(x) & \text { if } x \in(\zeta(t), 1]
\end{array} \quad \forall(x, t) \in[0,1] \times[0, T],\right.
$$

where we require of the "free boundary" $\zeta:[0, T] \rightarrow[0,1]$ that

$$
\zeta \in \mathrm{C}^{1}(0, T) \text { and is strictly increasing, with } \zeta(0)=0 .
$$

In fact, we have $u \in W^{1,1}\left(0, T ; L^{1}(0,1)\right)$, with

$$
u_{t}(x, t):=\left\{\begin{array}{ll}
-\zeta^{\prime}(t) & \text { if } x \in(0, \zeta(t)), \\
0 & \text { if } x \in(\zeta(t), 1)
\end{array} \quad \text { for a.e. }(x, t) \in(0,1) \times(0, T),\right.
$$


so that $\left\|u_{t}(\cdot, t)\right\|_{1}=\zeta(t) \zeta^{\prime}(t)$ for a.e. $t \in(0, T)$. Now, (1.20) is trivially fulfilled for $x \in(\zeta(t), 1), t \in(0, T)$, hence it reduces to

$$
-\zeta(t) \zeta^{\prime}(t)+1-\zeta(t)=0 \quad t \in(0, T) .
$$

Namely, the function $u$ given by (7.4) solves the Cauchy problem $(1.20,7.3)$ if and only if $\zeta$ fulfills

$$
\zeta^{\prime}(t)=\frac{1}{\zeta(t)}-1 \quad \forall t \in(0, T), \quad \zeta(0)=0 .
$$

On the other hand, we may interpret the function $u$ as a curve $u:(0, T) \rightarrow$ $L^{1}(0,1)$ : in this setting, its metric derivative is computed via (7.2). Taking into account that

$$
\begin{aligned}
& |u(x, t+h)-u(x, t)| \\
& =\left\{\begin{array}{lll}
\zeta(t+h)-\zeta(t) & \text { for } x \in[0, \zeta(t)], \\
\zeta(t+h)-x & \text { for } x \in(\zeta(t), \zeta(t+h)], & \forall(x, t) \in[0,1] \times[0, T], \\
0 & \text { for } x \in(\zeta(t+h), 1]
\end{array}\right.
\end{aligned}
$$

formula (7.2) yields

$$
\left|u^{\prime}\right|_{1}(t)=\zeta(t) \zeta^{\prime}(t) \text { for a.e. } t \in(0, T)
$$

(indeed, in this case $\left|u^{\prime}\right|_{1}(t)$ coincides with the $L^{1}$-norm of the function $u_{t}(\cdot, t)$ for a.e. $t \in(0, T))$. Now, we calculate the energy $\mathcal{E}(1.17)$ along the curve $u$ and find

$$
\mathcal{E}(u(t))=\frac{(1-\zeta(t))^{2}}{6}(2 \zeta(t)-1) \quad \forall t \in[0, T],
$$

while, also thanks to the representation formula (5.13) in Lemma 5.3,

$$
\begin{aligned}
& \left|\partial^{-} \mathcal{E}\right|(u(t))=|\partial \mathcal{E}|(u(t)) \\
& =\frac{1}{2} \sup _{w \in L^{1}(0,1), w \neq 0} \frac{\left(\int_{\Omega}\left(u^{2}(x, t)-(u(x, t)+w(x))^{2} \mathrm{~d} x\right)^{+}\right.}{\|w\|_{1}} \\
& =\|u(\cdot, t)\|_{\infty}=1-\zeta(t) \quad \forall t \in[0, T] .
\end{aligned}
$$

In view of (7.7)-(7.9), with elementary calculations it is possible to see that, if (7.6) holds, the curve $u$ fulfills the metric formulation (2.31)-(2.32), the latter in fact with an equality sign.

Example 7.2. We let $\Omega=(-1,1)$ and choose as energy functional $\mathcal{E}: L^{1}(-1,1) \rightarrow$ $[0,+\infty]$ the Dirichlet integral

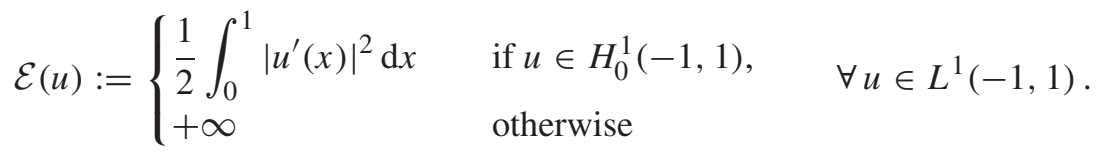


In fact, the above functional is a particular case of functional (7.19) below. The corresponding evolution equation is

$$
\left\|u_{t}(t)\right\|_{1} \operatorname{Sign}\left(u_{t}(x, t)\right)-u_{x x}(x, t) \ni 0 \quad \text { for a.e. }(x, t) \in(-1,1) \times(0, T),
$$

(where we denote by $u_{x}, u_{x x}$ the partial derivatives of $u$ with respect to the variable $x$ ), which we supplement with the initial datum

$$
u_{0}(x):=1-|x| \quad \forall x \in[-1,1] .
$$

We now look for a solution of the Cauchy problem (7.11)-(7.12) of the form

$$
u(x, t):=\left\{\begin{array}{ll}
\alpha(t)+c(t) \frac{x^{2}}{2} & \text { if }|x| \leq \zeta(t), \\
u_{0}(x) & \text { if } \zeta(t)<|x| \leq 1
\end{array} \quad \forall(x, t) \in[-1,1] \times[0, T],\right.
$$

under the requirements that $\zeta:[0, T] \rightarrow[0,1]$ complies with (7.5), the functions $\alpha, c \in \mathrm{C}^{1}(0, T), c$ takes strictly negative values and is strictly increasing, and for all $t \in(0, T)$

the maps $x \mapsto u(x, t), x \mapsto u_{t}(x, t), x \mapsto u_{x}(x, t)$ are continuous on $[-1,1]$.

Since for a.e. $(x, t) \in(-1,1) \times(0, T)$

$$
\begin{aligned}
& u_{t}(x, t):=\left\{\begin{array}{ll}
\alpha^{\prime}(t)+c^{\prime}(t) \frac{x^{2}}{2} & \text { if }|x|<\zeta(t), \\
0 & \text { if } \zeta(t)<|x|<1
\end{array},\right. \\
& u_{x}(x, t):=\left\{\begin{array}{ll}
c(t) x & \text { if }|x|<\zeta(t), \\
-\operatorname{Sign}(x) & \text { if } \zeta(t)<|x|<1
\end{array},\right.
\end{aligned}
$$

(7.14) leads to the conditions

$$
\alpha(t)+c(t) \frac{\zeta^{2}(t)}{2}=1-\zeta(t), \quad c(t)=-\frac{1}{\zeta(t)} \quad \forall t \in(0, T) .
$$

Hence, we compute

$$
\left\|u_{t}(\cdot, t)\right\|_{1}=\frac{c^{\prime}(t)}{2} \int_{-\zeta(t)}^{\zeta(t)}\left(\zeta^{2}(t)-x^{2}\right) \mathrm{d} x=\frac{2 c^{\prime}(t)}{3} \zeta^{3}(t) \quad \text { for a.e. } t \in(0, T)
$$

Being

$$
u_{x x}(x, t):=\left\{\begin{array}{ll}
c(t) & \text { if }|x|<\zeta(t), \\
0 & \text { if } \zeta(t)<|x|<1
\end{array} \quad \text { for a.e. }(x, t) \in(-1,1) \times(0, T),\right.
$$


and taking into account the second of (7.15), we conclude that $u$ solves (7.11) if and only if $c$ solves the Cauchy problem

$$
c(t)=\frac{3}{2} c^{4}(t) \quad \forall t \in(0, T), \quad \text { with } \lim _{t \searrow 0} c(t)=-\infty,
$$

so that

$$
c(t)=-\frac{9}{2} t^{-1 / 3}, \quad \zeta(t)=\frac{2}{9} t^{1 / 3}, \quad \alpha(t)=1-\frac{1}{9} t^{1 / 3} \quad \forall t \in(0, T) .
$$

From the metric viewpoint, the existence of a solution to the (Cauchy problem for) the metric formulation follows from Theorem 7.5 later on. Nonetheless, we may directly check that the function $u$ defined by (7.13) (seen as a curve on $(0, T)$ with values in $L^{1}(-1,1)$ ) complies with (2.31)-(2.32). Indeed, using (7.15) one easily checks that the metric derivative of $u$ again coincides with the $L^{1}(-1,1)$-norm of $u_{t}(\cdot, t)$ for a.e. $t \in(0, T)$, and it is thus given by (7.16). On the other hand, thanks to the forthcoming Lemma 7.3 we have that

$$
\left|\partial^{-} \mathcal{E}\right|(u(t))=|\partial \mathcal{E}|(u(t))=\left\|u_{x x}(\cdot, t)\right\|_{\infty} \quad \text { for a.e. } t \in(0, T) .
$$

Then, we calculate the energy (7.10) along the curve $u$ and, using (7.15) and (7.17) as well, we easily conclude that (2.32) holds, again as an equality.

\subsection{An existence result}

We consider the following energy functional $\mathcal{E}:[0, T] \times L^{1}(\Omega) \rightarrow(-\infty,+\infty]$, defined for all $(t, u) \in[0, T] \times L^{1}(\Omega)$ by

$$
\mathcal{E}_{t}(u):= \begin{cases}\int_{\Omega}\left(\frac{1}{2}|\nabla u(x)|^{2}+W(u(x))\right) \mathrm{d} x-\langle\ell(t), u\rangle & \text { if } u \in H_{0}^{1}(\Omega) \\ +\infty & \text { and } W(u) \in L^{1}(\Omega), \\ \text { otherwise. }\end{cases}
$$

Here, we suppose that

$$
\ell \in \mathrm{C}^{1}\left([0, T] ; H^{-1}(\Omega)\right) .
$$

and that the function $W$ fulfills

$$
W \in \mathrm{C}^{2}(\mathbb{R}) \quad \text { and } \exists C_{W}>0 \text { s.t. } \quad \forall r \in \mathbb{R} \quad W^{\prime \prime}(r) \geq-C_{W} ;
$$

for instance, one may think of the double-well potential

$$
W(u):=\frac{1}{4}\left(u^{2}-1\right)^{2} \quad \forall u \in \mathbb{R} .
$$

Note that functional $\mathcal{E}$ (7.19) is in fact a particular case of the class of functionals (8.22) which shall be tackled in Section 8.2 later on. The following result is crucial for understanding to which equation the metric formulation of Problem 2.6 (with the quadratic dissipation (1.16) and the energy (7.19)) leads. 


\section{Lemma 7.3.}

1. The functionals $\mathcal{E}_{t}$ are $\lambda$-uniformly convex on $L^{1}(\Omega)$ for some $\lambda<0$.

2. For every $(t, u) \in \operatorname{dom}(\mathcal{E})$

$\left|\partial \mathcal{E}_{t}\right|(u),\left|\partial^{-} \mathcal{E}_{t}\right|(u)<+\infty$ if and only if $-\Delta u+W^{\prime}(u)-\ell(t) \in L^{\infty}(\Omega)$.

In this case, $\quad\left|\partial \mathcal{E}_{t}\right|(u)=\left|\partial^{-} \mathcal{E}_{t}\right|(u)=\left\|-\Delta u+W^{\prime}(u)-\ell(t)\right\|_{\infty}$.

Proof of Claim 1. In order to check the convexity inequality (5.32), we fix $u_{0}, u_{1} \in$ $\operatorname{dom}(\mathcal{E}), \theta \in[0,1]$, and calculate

$$
\begin{aligned}
\mathcal{E}_{t}\left(u_{\theta}\right)= & \int_{\Omega}\left(\frac{1}{2}\left|\nabla u_{\theta}\right|^{2}+W\left(u_{\theta}\right)\right)-\left\langle\ell(t), u_{\theta}\right\rangle \\
\leq & \frac{1-\theta}{2} \int_{\Omega}\left|\nabla u_{0}\right|^{2}+\frac{\theta}{2} \int_{\Omega}\left|\nabla u_{1}\right|^{2}-\frac{\theta(1-\theta)}{2} \int_{\Omega}\left|\nabla\left(u_{0}-u_{1}\right)\right|^{2} \\
& +(1-\theta) \int_{\Omega} W\left(u_{0}\right)+\theta \int_{\Omega} W\left(u_{0}\right) \\
& +\frac{C_{W} \theta(1-\theta)}{2} \int_{\Omega}\left|u_{0}-u_{1}\right|^{2}-(1-\theta)\left\langle\ell(t), u_{0}\right\rangle-\theta\left\langle\ell(t), u_{1}\right\rangle \\
= & (1-\theta) \mathcal{E}_{t}\left(u_{0}\right)+\theta \mathcal{E}_{t}\left(u_{1}\right) \\
& +\frac{\theta(1-\theta)}{2} \int_{\Omega}\left(-\left|\nabla\left(u_{0}-u_{1}\right)\right|^{2}+C_{W}\left|u_{0}-u_{1}\right|^{2}\right),
\end{aligned}
$$

the first inequality following from the fact that $W$ itself is $\left(-C_{W}\right)$-convex ( $c f .(7.21))$. In order to estimate the remainder term on the right-hand side of (7.24), we apply the Gagliardo-Nirenberg inequality (see [44])

$$
\|v\|_{2} \leq C_{G N}\|v\|_{1}^{2 /(d+2)}\|\nabla v\|_{2}^{d /(d+2)} \quad \forall v \in H_{0}^{1}(\Omega),
$$

where $C_{G N}$ is a positive constant only depending on $\Omega$. Hence,

$$
\begin{aligned}
C_{W}\left\|u_{0}-u_{1}\right\|_{2}^{2}-\left\|\nabla\left(u_{0}-u_{1}\right)\right\|_{2}^{2} \leq & C_{W} C_{G N}^{2}\left\|u_{0}-u_{1}\right\|_{1}^{4 /(d+2)}\left\|\nabla\left(u_{0}-u_{1}\right)\right\|_{2}^{2 d /(d+2)} \\
& -\left\|\nabla\left(u_{0}-u_{1}\right)\right\|_{2}^{2} \\
\leq & C_{d}\left(C_{W} C_{G N}^{2}\right)^{(d+2) / 2}\left\|u_{0}-u_{1}\right\|_{1}^{2},
\end{aligned}
$$

for a positive constant $C_{d}$ only depending on $d$, the latter passage following from the Young inequality. Combining this estimate with (7.24), we deduce that the convexity inequality (5.32) holds with $\lambda=-C_{d}\left(C_{W} C_{G N}^{2}\right)^{(d+2) / 2}$.

Proof of Claim 2. Thanks to Claim 1 and to (5.15), it is sufficient to prove that for every $(t, u) \in \operatorname{dom}(\mathcal{E})$

$$
\begin{gathered}
\left|\partial \mathcal{E}_{t}\right|(u)<+\infty \Leftrightarrow-\Delta u+W^{\prime}(u)-\ell(t) \in L^{\infty}(\Omega), \text { and } \\
\left|\partial \mathcal{E}_{t}\right|(u)=\left\|-\Delta u+W^{\prime}(u)-\ell(t)\right\|_{\infty}
\end{gathered}
$$


Indeed, we set

$$
\mathcal{D}(u, w):=\frac{\left(\mathcal{E}_{t}(u)-\mathcal{E}_{t}(u+w)\right)^{+}}{\|w\|_{1}} \quad \text { for } u, w \in H_{0}^{1}(\Omega)
$$

and note that for all $w \in H_{0}^{1}(\Omega)$

$$
\mathcal{D}(u, r w) \rightarrow \mathcal{H}(u, w):=\frac{\left(\int_{\Omega}\left(-\nabla u \cdot \nabla w-W^{\prime}(u) w\right)+\langle\ell(t), w\rangle\right)^{+}}{\|w\|_{1}} \text { as } r \searrow 0 .
$$

Then, integrating by parts we find

$$
\left|\partial \mathcal{E}_{t}\right|(u) \geq \limsup _{r \searrow 0} \mathcal{D}(u, r w) \geq \frac{\left\langle\Delta u-W^{\prime}(u)+\ell(t), w\right\rangle}{\|w\|_{1}},
$$

so that, being $w$ arbitrary,

$$
\left|\partial \mathcal{E}_{t}\right|(u) \geq \sup _{w \in H_{0}^{1}(\Omega)} \frac{\left\langle\Delta u-W^{\prime}(u)+\ell(t), w\right\rangle}{\|w\|_{1}}=\left\|\Delta u-W^{\prime}(u)+\ell(t)\right\|_{\infty},
$$

the latter identity by the density of $H_{0}^{1}(\Omega)$ in $L^{1}(\Omega)$. On the other hand, we set

$$
\mathcal{G}(x, y):=W(x+y)-W(x)-W^{\prime}(x) y \quad \text { for } x, y \in \mathbb{R}
$$

and note that, by (7.21),

$$
-\mathcal{G}(x, y) \leq \frac{C_{W}}{2} y^{2} \quad \forall x, y \in \mathbb{R} .
$$

Now, trivial computations yield that

$$
\begin{aligned}
\left|\partial \mathcal{E}_{t}\right|(u) & =\limsup _{\|w\|_{1} \rightarrow 0} \mathcal{D}(u, w) \\
& \leq \limsup _{\|w\|_{1} \rightarrow 0} \mathcal{H}(u, w)+\limsup _{\|w\|_{1} \rightarrow 0} \frac{\left(-\frac{1}{2} \int_{\Omega}|\nabla w|^{2}-\int_{\Omega} \mathcal{G}(u, w)\right)^{+}}{\|w\|_{1}} .
\end{aligned}
$$

We have

$$
\begin{aligned}
& \limsup _{\|w\|_{1} \rightarrow 0} \frac{\left(-\frac{1}{2} \int_{\Omega}|\nabla w|^{2}-\int_{\Omega} \mathcal{G}(u, w)\right)^{+}}{\|w\|_{1}} \\
& \quad \leq \frac{1}{2} \limsup _{\|w\|_{1} \rightarrow 0} \frac{\left(-\|\nabla w\|_{2}^{2}+C_{W}\|w\|_{2}^{2}\right)^{+}}{\|w\|_{1}} \\
& \leq \frac{1}{2} \limsup _{\|w\|_{1} \rightarrow 0} \frac{\left(-\|\nabla w\|_{2}^{2}+C_{W} C_{G N}\|w\|_{1}^{4 /(d+2)}\|\nabla w\|_{2}^{2 d /(d+2)}\right)^{+}}{\|w\|_{1}} \\
& \leq \tilde{C} \limsup _{\|w\|_{1} \rightarrow 0} \frac{\|w\|_{1}^{2}}{\|w\|_{1}}=0
\end{aligned}
$$


the first passage following from (7.28), the second one from the GagliardoNirenberg inequality (7.25) and the last one by trivial calculations. Combining (7.29) and the above inequality, and again integrating by parts, we readily deduce the reverse inequality of (7.27), so that (7.26) follows.

Remark 7.4. In fact, the same argument as in the proof of Lemma 7.3 allows to prove that the Fréchet subdifferential of $\mathcal{E}$ has the following structure

$$
\begin{aligned}
& u \in \operatorname{dom}\left(\partial \mathcal{E}_{t}\right) \Leftrightarrow-\Delta u+W^{\prime}(u)-\ell(t) \in L^{\infty}(\Omega) \\
& \text { and in this case } \partial \mathcal{E}_{t}(u)=\left\{-\Delta u+W^{\prime}(u)-\ell(t)\right\} .
\end{aligned}
$$

We are now in the position of proving the following existence result.

Theorem 7.5. Assume (7.20), (7.21), and that

$$
W \text { is bounded from below on } \mathbb{R} \text {. }
$$

Then, for every $u_{0} \in H_{0}^{1}(\Omega)$ with $W\left(u_{0}\right) \in L^{1}(\Omega)$ there exists a solution $u \in$ $\mathrm{AC}\left(0, T ; L^{1}(\Omega)\right)$ of Problem 2.6 fulfilling $u(0)=u_{0}$, and we have the energy identity

$$
\begin{aligned}
\frac{1}{2} \int_{s}^{t}\left|u^{\prime}\right|_{1}^{2}(r) \mathrm{d} r+\frac{1}{2} \int_{s}^{t}\left\|-\Delta u(r)+W^{\prime}(u(r))-\ell(r)\right\|_{\infty}^{2} \mathrm{~d} r+\mathcal{E}_{t}(u(t)) \\
=\mathcal{E}_{s}(u(s))+\int_{s}^{t}\left\langle\ell^{\prime}(r), u(r)\right\rangle \mathrm{d} r \quad \forall 0 \leq s \leq t \leq T .
\end{aligned}
$$

Proof. It follows from Lemma 7.3 and from (7.20) that the functional $\mathcal{E}$ (7.19) yields an admissible family of functionals in the sense of Definition 5.10. Hence, thanks to Proposition $5.11 \mathcal{E}$ complies with the chain rule (5.36). Using (7.31) and arguing as in the proof of Lemma 8.10 later on, it is possible to check that the other assumptions on $\mathcal{E}$ (2.19a)-(2.19b), and (2.34)-(2.38) of Theorem 3.5 are satisfied. Then, the statement is a direct consequence of Theorem 3.5.

Remark 7.6. In fact, condition (7.31) on $W$ could be weakened, but here we prefer to keep the presentation as simple as possible, leaving to Section 8.2 the discussion of a more general example. In the particular case of the double well potential (7.22), we can infer some further regularity of the curve $u$ from (7.32). For instance, if $\ell \in L^{2}\left(0, T ; L^{p}(\Omega)\right)$ for some $1 \leq p<\infty$, we deduce that $-\Delta u+u^{3}-u \in$ $L^{2}\left(0, T ; L^{p}(\Omega)\right)$, hence by elliptic regularity $u \in L^{2}\left(0, T ; W_{0}^{2, p}(\Omega)\right)$.

\section{Quasivariational doubly nonlinear evolution equations in reflexive Banach spaces}

In this section, we deduce from our main Theorem 3.5 an existence result (Theorem 8.3 below) for a family of abstract quasivariational doubly nonlinear equations 
in the Banach space setup of Section 6. In particular, hereafter we shall assume that

$B$ is a reflexive and separable Banach space.

As an application, in Section 8.2 we prove the existence of solutions to initialboundary value problems for a class of doubly nonlinear parabolic evolution equations.

\subsection{A general existence result}

Throughout this section, besides (8.1) we assume that

$\eta_{u}: B \rightarrow[0,+\infty)$ is a family of convex and positively homogeneous

functionals, complying with (6.2), (6.3), (6.4),

and inducing the Finsler asymmetric distance $\Delta(6.6)$

$\mathcal{E}_{t}: B \rightarrow(-\infty,+\infty]$ is an admissible family of functionals according

to Definition 5.10, with sublevels locally compact with respect

to the strong topology of $B$ (cf. (2.38))

and the time derivative $\partial_{t} \mathcal{E}_{t}$ fulfils (2.37) with respect to $\Delta$;

$\psi:[0,+\infty) \rightarrow[0,+\infty]$ is a convex, lower semicontinuous function fulfilling (2.30), inducing the family of functionals

$$
\Psi_{u}(v):=\psi\left(\eta_{u}(v)\right) \in[0,+\infty], \quad \forall u, v \in B,
$$

\section{Statement of the problem}

We focus on the Cauchy problem

$$
\partial \Psi_{u(t)}\left(u^{\prime}(t)\right)+\partial \mathcal{E}_{t}(u(t)) \ni 0 \quad \text { for a.e. } t \in(0, T), \quad u(0)=u_{0},
$$

where $u_{0} \in \mathrm{D}$ is some initial datum and $u \in \mathrm{AC}(0, T ; B)$.

This problem admits the following metric formulation, where $\left|u^{\prime}\right|$ and $\left|\partial^{-} \mathcal{E}_{t}\right|(u)$ respectively denote the metric velocity and the (relaxed) metric slope induced by the asymmetric distance $\Delta$ :

Problem 8.1. Find a curve $u \in \mathrm{AC}(0, T ; B)$ such that

$$
\begin{gathered}
u(0)=u_{0}, \text { the map } t \mapsto \mathcal{E}_{t}(u(t)) \text { is absolutely continuous on }(0, T) \text {, and } \\
\frac{d}{d t} \mathcal{E}_{t}(u(t))-\partial_{t} \mathcal{E}_{t}(u(t)) \leq-\psi\left(\left|u^{\prime}\right|(t)\right)-\psi^{*}\left(\left|\partial^{-} \mathcal{E}_{t}\right|(u(t))\right) \quad \text { for a.e. } t \in(0, T) .
\end{gathered}
$$

In the sequel, we shall first of all investigate to which extent a solution $u$ to Problem 8.1 turns out to be a solution of the Cauchy problem (8.2). Secondly, we shall deduce from the "metric" existence Theorem 3.5 an existence result for (8.2). 


\section{Links between the metric and the Banach space formulation}

We have the following result, which extends [5, Proposition 1.4.1] to the doubly nonlinear setting.

Proposition 8.2. Let $u_{0} \in \mathrm{D}$ and $u \in \mathrm{AC}(0, T ; B)$ fulfill (8.3). Then, $u$ solves the Cauchy problem (8.2). In particular, there holds

$$
\partial \Psi_{u(t)}\left(u^{\prime}(t)\right) \supset \operatorname{Argmin}\left\{\eta_{u(t) *}(-\xi): \xi \in \partial \mathcal{E}_{t}(u(t))\right\} \text { for a.e. } t \in(0, T) .
$$

Conversely, if $u$ solves (8.2) and if the map $t \mapsto \mathcal{E}_{t}(u(t))$ is absolutely continuous on $(0, T)$, then $u$ also fulfills (8.3).

Proof. Suppose that $u \in \mathrm{AC}(0, T ; B)$ fulfills (8.3): then, there exists a negligible set $\mathcal{N} \subset(0, T)$ such that for all $t \in(0, T) \backslash \mathcal{N}$ the derivatives $\frac{d}{d t} \mathcal{E}_{t}(u(t))$ and $\left|u^{\prime}\right|(t)=\eta_{u(t)}\left(u^{\prime}(t)\right)$ (see Theorem 6.2) exist, with $\left|\partial^{-} \mathcal{E}_{t}\right|(u(t)) \geq \min _{\xi \in \partial \mathcal{E}_{t}(u(t))} \eta_{u(t) *}(-\xi)$ by Corollary 6.6. Hence, (8.3) yields

$$
\begin{aligned}
& \frac{d}{d t} \mathcal{E}_{t}(u(t))-\partial_{t} \mathcal{E}_{t}(u(t)) \leq-\psi\left(\eta_{u(t)}\left(u^{\prime}(t)\right)\right)-\psi^{*}\left(\eta_{u(t) *}(-\xi)\right) \\
& \forall \xi \in \operatorname{Argmin}\left\{\eta_{u(t) *}(-\xi): \xi \in \partial \mathcal{E}_{t}(u(t))\right\} \quad \forall t \in(0, T) \backslash \mathcal{N} .
\end{aligned}
$$

Combining this inequality with (6.26), we deduce that for a.e. $t \in(0, T)$

$$
\begin{gathered}
\eta_{u(t)}\left(u^{\prime}(t)\right) \cdot \eta_{u(t) *}(-\xi)=\psi\left(\eta_{u(t)}\left(u^{\prime}(t)\right)+\psi^{*}\left(\eta_{u(t) *}(-\xi)\right), \quad\right. \text { whence } \\
\eta_{u(t) *}(-\xi) \in \partial \psi\left(\eta_{u(t)}\left(u^{\prime}(t)\right)\right) \quad \forall \xi \in \operatorname{Argmin}\left\{\eta_{u(t) *}(-\xi): \xi \in \partial \mathcal{E}_{t}(u(t))\right\} .
\end{gathered}
$$

Thanks to Lemma 5.1, we conclude (8.4).

The second part of the statement follows by the same argument.

\section{An existence result}

The following Theorem 8.3 extends [5, Theorem 2.3.7], of which we closely follow the proof.

Theorem 8.3. Under assumptions (8.1), $(\mathrm{N}),(\mathrm{E})$, and $(\Psi)$, for every $u_{0} \in \mathrm{D}$ there exists a curve $u \in \operatorname{AC}(0, T ; B)$, with $u(0)=u_{0}$, satisfying the differential inclusion (8.4). Moreover, u fulfils the energy identity

$$
\begin{array}{r}
\int_{s}^{t} \Psi_{u(r)}\left(u^{\prime}(r)\right) \mathrm{d} r+\int_{s}^{t} \psi^{*}\left(\left|\partial^{-} \mathcal{E}_{r}\right|(u(r))\right) \mathrm{d} r+\mathcal{E}_{t}(u(t)) \\
=\mathcal{E}_{s}(u(s))+\int_{s}^{t} \partial_{t} \mathcal{E}_{r}(u(r)) \mathrm{d} r \quad \forall 0 \leq s \leq t \leq T .
\end{array}
$$


Proof. It is straightforward to check that the functionals $\mathcal{E}$ and $\psi$ comply with all the assumptions of Theorem 3.5 (in particular, the chain rule of Definition 2.5 holds thanks to Theorem 6.7). Then, there exists a solution $u \in \mathrm{AC}(0, T ; B)$ to (8.3), fulfilling the energy identity (3.9). By Proposition 8.2, $u$ solves (8.4), while, in view of (6.8), the energy identity (3.9) yields (8.5).

\subsection{Applications to doubly nonlinear parabolic evolutions}

\section{Setup of the problem}

In the sequel, we shall examine the following evolution equation ( $c f$. with (1.15))

$$
\rho_{\operatorname{sign}\left(u_{t}\right)}(u)\left|u_{t}\right|^{p-2} u_{t}-\operatorname{div}(\beta(\nabla u))+W^{\prime}(u)=h \quad \text { in } \Omega \times(0, T),
$$

Here, $1<p<\infty, \Omega \subset \mathbb{R}^{d}, d \geq 1$, is a bounded domain with sufficiently smooth boundary and exterior unit normal $\mathbf{n}$. Further, we are given two functions $\rho_{+}, \rho_{-}: \mathbb{R} \rightarrow(0,+\infty)$, and we adopt the following notation

$$
\rho_{\operatorname{sign}(v)}(u)=\left\{\begin{array}{ll}
\rho_{+}(u) & \text { if } v \geq 0, \\
\rho_{-}(u) & \text { if } v<0
\end{array} \quad \forall u, v \in \mathbb{R} .\right.
$$

Moreover, $\beta: \mathbb{R}^{d} \rightarrow \mathbb{R}^{d}$ is the gradient of some smooth function $j$ on $\mathbb{R}^{d}, W$ : $\mathbb{R} \rightarrow \mathbb{R}$ a differentiable function and $h: \Omega \times(0, T) \rightarrow \mathbb{R}$ some source term. In particular, when $\beta(\zeta)=|\zeta|^{q-2} \zeta$ for some $q>1$, the elliptic operator in (8.6) is indeed the $q$-Laplacian and we recover (1.15).

We consider the following initial-boundary value problem for (8.6).

Problem 8.4. Given $u_{0} \in L^{p}(\Omega)$, find a function $u \in W^{1, p}\left(0, T ; L^{p}(\Omega)\right)$ satisfying (8.6) a.e. on $\Omega \times(0, T)$, the homogeneous Dirichlet boundary condition

$$
u=0 \quad \text { a.e. in } \partial \Omega \times(0, T),
$$

and the initial condition

$$
u(x, 0)=u_{0}(x) \text { for a.e. } x \in \Omega .
$$

\section{Further notation}

Before stating our existence result for Problem 8.4, let us fix some notation. For a fixed $q \in(1,+\infty)$ we set

$$
q^{\star}:= \begin{cases}\frac{d q}{d-q} & \text { if } q \in(1, d), \\ +\infty & \text { if } q \geq d .\end{cases}
$$

Henceforth, we shall consider on the space $W_{0}^{1, q}(\Omega)$ the norm $\|u\|_{1, q}:=\|\nabla u\|_{q}$ for all $u \in W_{0}^{1, q}(\Omega)$ (equivalent to the usual Sobolev norm by the Poincaré inequality); 
we shall denote by $\|\cdot\|_{-1, q^{\prime}}$ the norm of the dual space $W^{-1, q^{\prime}}(\Omega)\left(q^{\prime}\right.$ being the conjugate exponent of $q$ ), and by $\langle\cdot, \cdot\rangle$ the duality pairing between $W^{-1, q^{\prime}}(\Omega)$ and $W_{0}^{1, q}(\Omega)$. It is well known (see, e.g., [1]) that

$$
W_{0}^{1, q}(\Omega) \subset L^{q^{\star}}(\Omega) \text { and } \begin{cases}W_{0}^{1, q}(\Omega) \subset \subset L^{q^{\star}-\varepsilon}(\Omega) \quad \forall \varepsilon>0 & \text { if } d>q, \\ W_{0}^{1, q}(\Omega) \subset \subset L^{r}(\Omega) \quad \forall 1 \leq r<\infty & \text { if } d=q, \\ W_{0}^{1, q}(\Omega) \subset \subset L^{\infty}(\Omega) & \text { if } d<q .\end{cases}
$$

Finally, we shall denote by $\mathrm{C}_{\mathrm{w}}^{0}\left([0, T] ; W_{0}^{1, q}(\Omega)\right)$ the space of weakly continuous functions with values in $W_{0}^{1, q}(\Omega)$.

\section{An existence result}

Let us enlist our main assumptions on the data of Problem 8.4:

$$
\begin{aligned}
& \text { the functions } \rho_{+}, \rho_{-}: \mathbb{R} \rightarrow(0,+\infty) \quad \text { are continuous, and } \\
& \quad \exists R_{0}, R_{1}>0: \quad R_{0} \leq \rho_{-}(x), \rho_{+}(x) \leq R_{1} \quad \forall x \in \mathbb{R} ;
\end{aligned}
$$

there exists a function $j \in \mathrm{C}^{1}\left(\mathbb{R}^{d}\right)$ such that $\beta=\nabla j: \mathbb{R}^{d} \rightarrow \mathbb{R}^{d}$ and

$$
\begin{gathered}
\exists 1<q<\infty \text { with } p<q^{\star}, \quad \exists M_{1}, M_{2}, M_{3}>0: \\
\left\{\begin{array}{l}
j(\zeta) \geq M_{1}|\zeta|^{q}-M_{2}, \quad \forall \zeta \in \mathbb{R}^{d} ; \\
|\beta(\zeta)| \leq M_{3}\left(1+|\zeta|^{q-1}\right)
\end{array}\right.
\end{gathered}
$$

further, we have

$$
W=W_{c}+g, \quad \text { where }
$$

$W_{c}$ is a convex and differentiable function, and

$$
g \in C^{1}(\mathbb{R}) \text { satisfies the growth conditions: }
$$

$$
\exists \alpha>0 \text { with } \alpha p^{\prime}<q^{\star}, \exists M_{4}>0:\left|g^{\prime}(u)\right| \leq M_{4}\left(|u|^{\alpha}+1\right) \quad \forall u \in \mathbb{R} .
$$

Finally, we require that

$$
h \in \mathrm{C}^{1}\left([0, T] ; W^{-1, q^{\prime}}(\Omega)\right), \quad u_{0} \in W_{0}^{1, q}(\Omega), \quad \text { and } \quad W_{c}\left(u_{0}\right) \in L^{1}(\Omega) .
$$

Theorem 8.5. Assume (8.11)-(8.14). Then, Problem 8.4 admits a solution

$$
\begin{gathered}
u \in W^{1, p}\left(0, T ; L^{p}(\Omega)\right) \cap L^{\infty}\left(0, T ; W_{0}^{1, q}(\Omega)\right) \subset \mathrm{C}_{\mathrm{w}}^{0}\left([0, T] ; W_{0}^{1, q}(\Omega)\right), \\
\text { with } g^{\prime}(u) \in L^{\infty}\left(0, T ; L^{p^{\prime}}(\Omega)\right) .
\end{gathered}
$$

Furthermore, if $h \in L^{p^{\prime}}\left(0, T ; L^{p^{\prime}}(\Omega)\right)$ as well, then $u$ has the further regularity

$$
-\operatorname{div}(\beta(\nabla u))+W_{c}^{\prime}(u) \in L^{p^{\prime}}\left(0, T ; L^{p^{\prime}}(\Omega)\right) .
$$


Remark 8.6. Let us point out that, if $q \geq d$, condition (8.13) allows the non convex part of the potential $g$ to have any polynomial growth at infinity. Furthermore, note that, in the case $d=3$ and $p=q=2$, the double well potential $W(u):=$ $\left(u^{2}-1\right)^{2} / 4$ fits in this framework. In fact, in that case the non convex function $g$ is allowed to have a polynomial growth of order $4-\varepsilon$ for all $\varepsilon>0$.

Remark 8.7. Slight modifications in the assumptions, which we are not going to detail, would also allow us to prove an existence result for Problem 8.4 with homogeneous Neumann boundary conditions on $u$.

We shall prove Theorem 8.5 by going over to the formulation of Problem 8.4 as a doubly nonlinear evolution inclusion of the type (8.2) in the (reflexive) Banach space

$$
B=L^{p}(\Omega), \quad 1<p<\infty,
$$

endowed with a suitable Finsler metric.

\section{The Banach space formulation}

In order to introduce the formulation of Problem 8.4 as a doubly nonlinear equation in $L^{p}(\Omega)$, we consider the function $\mathcal{R}: \mathbb{R}^{2} \rightarrow[0,+\infty)$ given by

$$
\mathcal{R}(w, z):=\left\{\begin{array}{ll}
\rho_{+}(w) z^{p} & \text { if } z \geq 0, \\
\rho_{-}(w)|z|^{p} & \text { if } z<0
\end{array}=\rho_{\operatorname{sign}(z)}(w)|z|^{p} \quad \forall(w, z) \in \mathbb{R}^{2} .\right.
$$

We associate with $\mathcal{R}$ the following family of positive functionals on $L^{p}(\Omega)$ :

$$
\eta_{u}(v):=\left(\int_{\Omega} \mathcal{R}(u(x), v(x)) \mathrm{d} x\right)^{1 / p} \quad \forall u, v \in L^{p}(\Omega) .
$$

Further, let us consider the function $\psi(x):=\frac{x^{p}}{p}, x \geq 0$, inducing (see $(\Psi)$ ) the functionals

$$
\Psi_{u}(v):=\frac{1}{p}\left(\eta_{u}(v)\right)^{p}=\frac{1}{p} \int_{\Omega} \mathcal{R}(u(x), v(x)) \mathrm{d} x \quad \forall u, v \in L^{p}(\Omega) .
$$

Finally, let us define $\mathcal{E}^{1}:[0, T] \times L^{p}(\Omega) \rightarrow(-\infty,+\infty]$ by

$$
\mathcal{E}_{t}^{1}(u):= \begin{cases}\int_{\Omega}\left(j(\nabla u(x))+W_{c}(u(x))\right) \mathrm{d} x-\langle h(t), u\rangle & \text { if } u \in W_{0}^{1, q}(\Omega) \\ +\infty & \text { and } W_{c}(u) \in L^{1}(\Omega), \\ \text { otherwise }\end{cases}
$$

for all $(t, u) \in[0, T] \times L^{p}(\Omega)$, and $\mathcal{E}^{2}:[0, T] \times L^{p}(\Omega) \rightarrow(-\infty,+\infty]$ by

$$
\mathcal{E}_{t}^{2}(u):= \begin{cases}\int_{\Omega} g(u(x)) \mathrm{d} x & \text { if } g(u) \in L^{1}(\Omega), \quad \forall(t, u) \in[0, T] \times L^{p}(\Omega), \\ +\infty & \text { otherwise }\end{cases}
$$


and let us set

$$
\mathcal{E}_{t}(u):=\mathcal{E}_{t}^{1}(u)+\mathcal{E}_{t}^{2}(u) \quad \forall(t, u) \in[0, T] \times L^{p}(\Omega) .
$$

We have the following

Proposition 8.8. Assume (8.11)-(8.14). Then, every solution $u \in \mathrm{AC}\left(0, T ; L^{p}(\Omega)\right)$ of the Cauchy problem (8.2) associated with the functionals $\left\{\Psi_{u}\right\}_{u \in L^{p}(\Omega)}$ and $\mathcal{E}_{t}$ : $L^{p}(\Omega) \rightarrow(-\infty,+\infty], t \in[0, T]$, respectively given by (8.19) and (8.22), is a solution of Problem 8.4.

The proof of Proposition 8.8 ensues from the following results, which shed light on the properties of the functionals $\left\{\eta_{u}\right\}_{u \in L^{p}(\Omega)}(8.18)$ and $\mathcal{E}_{t}(8.22)$.

Lemma 8.9. Under assumption (8.11), $\left\{\eta_{u}\right\}_{u \in L^{p}(\Omega)}$ is a family of sublinear functionals complying with (6.2), (6.3), (6.4), and for all $u \in L^{p}(\Omega)$ we have

$$
\eta_{u *}(\xi)=\left(\int_{\Omega} \mathcal{R}_{*}(u(x), \xi(x)) \mathrm{d} x\right)^{1 / p^{\prime}} \quad \forall \xi \in L^{p^{\prime}}(\Omega)
$$

where $\mathcal{R}_{*}: \mathbb{R}^{2} \rightarrow(0,+\infty)$ is defined by

$$
\mathcal{R}_{*}(w, z):=\left\{\begin{array}{ll}
\rho_{+}(w)^{-p^{\prime} / p} z^{p^{\prime}} & \text { if } z \geq 0 \\
\rho_{-}(w)^{-p^{\prime} / p}|z|^{p^{\prime}} & \text { if } z<0
\end{array}=\rho_{\operatorname{sign}(z)}^{-p^{\prime} / p}(w)|z|^{p^{\prime}} \quad \forall(w, z) \in \mathbb{R}^{2} .\right.
$$

Further, for all $u \in L^{p}(\Omega)$ and $v \in \operatorname{dom}\left(\partial \Psi_{u}\right)$ we have

$\xi \in \partial \Psi_{u}(v)\left(\subset L^{p^{\prime}}(\Omega)\right) \quad \Leftrightarrow \quad \xi(x)=\rho_{\operatorname{sign}(v(x))}(u(x)) v(x)^{p-1} \quad$ for a.e. $x \in \Omega$.

Note that, thanks to (8.11),

$$
R_{1}^{-p^{\prime} / p}|z|^{p^{\prime}} \leq \mathcal{R}_{*}(w, z) \leq R_{0}^{-p^{\prime} / p}|z|^{p^{\prime}} \quad \forall(w, z) \in \mathbb{R}^{2} .
$$

Proof of Lemma 8.9. Conditions (5.1a) and (5.1c) (with $K$ independent of $u$ ) are trivial to check.

Concerning (5.1b), let us first note that

$$
\mathcal{R}\left(s, t_{1}+t_{2}\right) \leq\left(\mathcal{R}\left(s, t_{1}+t_{2}\right)\right)^{(p-1) / p} \cdot\left(\mathcal{R}\left(s, t_{1}\right)^{1 / p}+\mathcal{R}\left(s, t_{2}\right)^{1 / p}\right) \quad \forall s, t_{1}, t_{2} \in \mathbb{R} .
$$

Indeed, to fix ideas let us suppose that $t_{1}+t_{2} \geq 0$ (the other case can be treated exactly in the same way). Then,

$$
\begin{aligned}
\mathcal{R}\left(s, t_{1}+t_{2}\right)= & \rho_{+}(s)\left(t_{1}+t_{2}\right)^{p} \\
= & \rho_{+}(s)^{1 / p} t_{1} \cdot\left(\rho_{+}(s)^{(p-1) / p} \cdot\left(t_{1}+t_{2}\right)^{p-1}\right) \\
& +\rho_{+}(s)^{1 / p} t_{2}\left(\rho_{+}(s)^{(p-1) / p} \cdot\left(t_{1}+t_{2}\right)^{p-1}\right) .
\end{aligned}
$$


If $t_{1}, t_{2} \geq 0$, (8.26) follows. If, e.g., $t_{1} \geq 0$ and $t_{2} \leq 0$, using that $\rho_{+}(s)^{1 / p} t_{2} \leq$ $0 \leq \rho_{-}(s)^{1 / p}\left|t_{2}\right|$ we again deduce (8.26). Therefore, by the Hölder inequality we have for all $u, v_{1}, v_{2} \in L^{p}(\Omega)$ :

$$
\begin{aligned}
\eta_{u}\left(v_{1}+v_{2}\right)^{p} & =\int_{\Omega} \mathcal{R}\left(u(x), v_{1}(x)+v_{2}(x)\right) \mathrm{d} x \\
& \leq \int_{\Omega} \mathcal{R}\left(u(x), v_{1}(x)\right)^{1 / p} \cdot\left(\mathcal{R}\left(u(x), v_{1}(x)+v_{2}(x)\right)\right)^{(p-1) / p} \mathrm{~d} x \\
& +\int_{\Omega} \mathcal{R}\left(u(x), v_{2}(x)\right)^{1 / p} \cdot\left(\mathcal{R}\left(u(x), v_{1}(x)+v_{2}(x)\right)\right)^{(p-1) / p} \mathrm{~d} x \\
& \leq \eta_{u}\left(v_{1}\right) \cdot \eta_{u}\left(v_{1}+v_{2}\right)^{(p-1) / p}+\eta_{u}\left(v_{2}\right) \cdot \eta_{u}\left(v_{1}+v_{2}\right)^{(p-1) / p},
\end{aligned}
$$

whence (5.1b).

We shall now prove that for all $\left\{u_{n}\right\},\left\{v_{n}\right\} \subset L^{p}(\Omega)$

$$
\left(u_{n} \rightarrow u, v_{n} \rightarrow v \text { in } L^{p}(\Omega)\right) \Rightarrow \eta_{u_{n}}\left(v_{n}\right) \rightarrow \eta_{u}(v) \quad \text { as } n \uparrow \infty
$$

which clearly implies (6.4). Indeed, there exist two subsequences $\left\{u_{n_{k}}\right\}$ and $\left\{v_{n_{k}}\right\}$ such that $u_{n_{k}} \rightarrow u$ and $v_{n_{k}} \rightarrow v$ a.e. on $\Omega$. Then it can be easily checked that

$$
\mathcal{R}\left(u_{n_{k}}(x), v_{n_{k}}(x)\right) \rightarrow \mathcal{R}(u(x), v(x)) \text { for a.e. } x \in \Omega \text {. }
$$

From (8.11) we infer that

$$
\begin{aligned}
\mathcal{R}\left(u_{n_{k}}(x), v_{n_{k}}(x)\right) & \leq R_{1}^{p}\left|v_{n_{k}}(x)\right|^{p} \\
& \leq 2^{p-1} R_{1}^{p}\left(\left|v_{n_{k}}(x)-v(x)\right|^{p}+|v(x)|^{p}\right) \quad \text { for a.e. } x \in \Omega .
\end{aligned}
$$

Using a generalized version of the Lebesgue theorem (see e.g. [23]), we deduce that $\eta_{u_{n_{k}}}\left(v_{n_{k}}\right) \rightarrow \eta_{u}(v)$ as $k \uparrow \infty$. As the limit does not depend on the extracted subsequence, (8.27) follows.

Further, let $\left\{u_{n}\right\}$ and $\left\{v_{n}\right\}$ fulfill $u_{n} \rightarrow u$ and $v_{n} \rightarrow v$ in $L^{p}(\Omega)$. Again applying the aforementioned lower semicontinuity results [11, Theorem 3.2] or [35, Theorem B.1] to the functional $(u, v) \mapsto \mathcal{R}(u, v)$, we deduce that

$$
\liminf _{n \uparrow \infty} \int_{\Omega} \mathcal{R}\left(u_{n}(x), v_{n}(x)\right) \mathrm{d} x \geq \int_{\Omega} \mathcal{R}(u(x), v(x)) \mathrm{d} x,
$$

whence the lower semicontinuity property (6.3).

Finally, (8.23) follows from trivial computations and, in order to check (8.24), we fix $u \in L^{p}(\Omega)$ and $v \in \operatorname{dom}\left(\partial \Psi_{u}\right)$, supposing without loss of generality that 
$\eta_{u}(v) \neq 0$ (if $\eta_{u}(v)=0$, necessarily $v=0$ and the check of (8.24) simplifies). By Lemma 5.1 and the definition (8.19) of $\Psi_{u}$, we have

$$
\begin{aligned}
\xi \in \partial \Psi_{u}(v) & \Leftrightarrow \eta_{u *}(\xi)=\eta_{u}(v)^{p-1} \text { and } \eta_{u *}(\xi)=\frac{\int_{\Omega} \xi(x) v(x) \mathrm{d} x}{\eta_{u}(v)} \\
& \Leftrightarrow\left(\int_{\Omega} \mathcal{R}(u(x), v(x)) \mathrm{d} x\right)^{(p-1) p}=\eta_{u *}(\xi)=\frac{\int_{\Omega} \xi(x) v(x) \mathrm{d} x}{\left(\int_{\Omega} \mathcal{R}(u(x), v(x)) \mathrm{d} x\right)^{1 / p}} \\
& \Leftrightarrow \xi(x)=\rho_{\operatorname{sign}(v(x))}(u(x))|v(x)|^{p-2} v(x) \quad \text { for a.e. } x \in \Omega .
\end{aligned}
$$

Lemma 8.10. Assume (8.12)-(8.14). Then, the functional $\mathcal{E}:[0, T] \times L^{p}(\Omega)$ defined by (8.22) yields an admissible family of functionals (according to Definition 5.10), fulfilling condition (E) of Section 8.1. Furthermore, for all $t \in[0, T]$ the Fréchet subdifferential $\partial \mathcal{E}_{t}(u) \neq \emptyset$ if and only if $-\operatorname{div}(\beta(\nabla(u)))+W^{\prime}(u)-h(t) \in$ $L^{p^{\prime}}(\Omega)$ and, in that case,

$$
\partial \mathcal{E}_{t}(u):=\left\{-\operatorname{div}(\beta(\nabla(u)))+W^{\prime}(u)-h(t)\right\} .
$$

Proof. Hereafter, we focus on the case in which $q<d$, as the proof in the other case is analogous and slightly simpler. Note that

$$
\operatorname{dom}\left(\mathcal{E}_{t}\right)=\mathrm{D}=\left\{u \in W_{0}^{1, q}(\Omega): W_{c}(u) \in L^{1}(\Omega)\right\} \quad \forall t \in[0, T] .
$$

It follows from [26, Theorem 2.5, p. 22] that for all $t \in[0, T]$ the functional $\mathcal{E}_{t}^{1}$ is convex and lower semicontinuous. Moreover, recalling that

$$
\exists l_{1}, l_{2}>0: \quad W_{c}(u) \geq-l_{1} u-l_{2} \quad \forall u \in \mathbb{R},
$$

we find that

$$
\begin{aligned}
\mathcal{E}_{t}^{1}(u) & \geq M_{1}\|\nabla u\|_{q}^{q}-l_{1}\|u\|_{1}-\|h(t)\|_{-1, q^{\prime}}\|u\|_{1, q}-C \\
& \geq \frac{M_{1}}{2}\|u\|_{1, q}^{q}-C\|h\|_{L^{\infty}\left(0, T ; W^{-1, q^{\prime}}(\Omega)\right)}^{q^{\prime}}-C^{\prime}
\end{aligned}
$$

for all $(t, u) \in[0, T] \times W_{0}^{1, q}(\Omega)$, due to (8.12) and an elementary application of the Young inequality. Hence, the functionals $\mathcal{E}_{t}^{1}$ are uniformly bounded from below with respect to $t$. Arguing as in [46], it can be readily checked that for all $(t, u) \in[0, T] \times W_{0}^{1, q}(\Omega)$

$\partial \mathcal{E}_{t}^{1}(u)= \begin{cases}\left\{-\operatorname{div}(\nabla \beta(u))+W_{c}^{\prime}(u)-h(t)\right\} & \text { if } \operatorname{div}(\nabla \beta(u))-W_{c}^{\prime}(u)+h(t) \in L^{p^{\prime}}(\Omega), \\ \varnothing & \text { otherwise. }\end{cases}$ 
On the other hand, one trivially sees that the functional $\mathcal{E}^{2}$ is lower semicontinuous; further, using the growth condition for $g^{\prime}$ in (8.13), the Sobolev embedding (8.10), as well as the Hölder and the Young inequalities, one has for all $v>0$

$$
\begin{aligned}
\left|\mathcal{E}_{t}^{2}(u)\right| & \leq M_{4} \int_{\Omega}|u(x)|^{\alpha+1} \mathrm{~d} x+C \leq M_{4}\left\||u|^{\alpha}\right\|_{p^{\prime}}\|u\|_{p}+C \\
& \leq C^{\prime}\|u\|_{q^{\star}}\|u\|_{p}+C \leq v\|u\|_{q^{\star}}^{q}+C_{v}\|u\|_{p}^{q^{\prime}}+C,
\end{aligned}
$$

so that (5.19) follows by combining the above estimate with (8.29) and choosing $v$ in such a way that $2 v / M_{1}<1$. Moreover, (8.29) yields that the sublevels of $\mathcal{E}_{t}(\cdot)$ are bounded in $W_{0}^{1, q}(\Omega)$ (which is compactly embedded in $L^{p}(\Omega)$ ), uniformly with respect to $t \in[0, T]$, hence $(2.38)$ is fulfilled.

Let us now check that $\mathcal{E}_{t}^{2}$ fulfills the "differentiability" property (5.20) with

$$
\widetilde{\mathrm{D}} \mathcal{E}_{t}^{2}(u)=g^{\prime}(u) \quad \forall u \in W_{0}^{1, q}(\Omega) \quad \forall t \in[0, T] .
$$

Indeed, arguing as above we see that by (8.13) and (8.29) there exists a positive constant $M_{5}$ such that for all $u \in W_{0}^{1, q}(\Omega)$

$g^{\prime}(u) \in L^{p^{\prime}}(\Omega)$ and $\left\|g^{\prime}(u)\right\|_{p^{\prime}} \leq C\left(\|u\|_{q^{\star}}^{q^{\star}}+1\right)^{1 / p^{\prime}} \leq M_{5}\left(\mathcal{E}_{t}^{1}(u)^{q^{\star} / q p^{\prime}}+1\right)$.

In order to check (5.20), let us fix a sequence $\left\{u_{n}\right\} \subset W_{0}^{1, q}(\Omega)$ fulfilling $\sup _{n} \mathcal{E}_{t}\left(u_{n}\right)$ and converging to $u$ in $L^{p}(\Omega)$ : it follows from (8.29) and from (8.10) that

$$
u_{n} \rightarrow u \quad \text { in } L^{q^{\star}-\varepsilon}(\Omega) \text { for all } \varepsilon>0 .
$$

By the mean value theorem, for a.e. $x \in \Omega$

$$
g\left(u_{n}(x)\right)-g(u(x))=\left(\int_{0}^{1} g^{\prime}\left((1-t) u_{n}(x)+t u(x)\right) \mathrm{d} t\right)\left(u_{n}(x)-u(x)\right) .
$$

Therefore,

$$
\begin{aligned}
& \lim _{n \uparrow \infty} \frac{\left|\int_{\Omega} g\left(u_{n}(x)\right)-g(u(x))-g^{\prime}(u(x))\left(u_{n}(x)-u(x)\right) \mathrm{d} x\right|}{\left\|u_{n}-u\right\|_{p}} \\
& \leq \lim _{n \uparrow \infty} \frac{\int_{\Omega}\left|\int_{0}^{1}\left(g^{\prime}\left((1-t) u_{n}(x)+t u(x)\right)-g^{\prime}(u(x))\right) \mathrm{d} t\right|\left|u_{n}(x)-u(x)\right| \mathrm{d} x}{\left\|u_{n}-u\right\|_{p}} \\
& \leq \lim _{n \uparrow \infty} \int_{0}^{1}\left\|g^{\prime}\left((1-t) u_{n}+t u\right)-g^{\prime}(u)\right\|_{p^{\prime}} \mathrm{d} t .
\end{aligned}
$$


the first inequality following from (8.34) and the second one from the Hölder inequality. Using the growth condition (8.13), (8.33) and a version of the Dominated Convergence theorem (see [23]), we infer that

$$
\int_{0}^{1}\left\|g^{\prime}\left((1-t) u_{n}+t u\right)-g^{\prime}(u)\right\|_{p^{\prime}} \mathrm{d} t \rightarrow 0 \quad \text { as } n \uparrow \infty,
$$

hence (8.31) follows. Then, (5.21) follows from (8.32). Noting that $\partial_{t} \mathcal{E}_{t}(u)=$ $-\left\langle h^{\prime}(t), u\right\rangle$ for every $u \in W_{0}^{1, q}(\Omega)$, we readily conclude from the previous computations that (5.34) holds. Hence, (2.37) follows from the fact that the sublevels of $\mathcal{E}_{t}$ are weakly compact in $W_{0}^{1, q}(\Omega)$ and that $h^{\prime} \in \mathrm{C}^{0}\left([0, T] ; W^{-1, q^{\prime}}(\Omega)\right.$ ). Finally, (8.28) follows from the representation formula (5.23) of the Fréchet subdifferential of admissible functionals, joint with (8.30) and (8.31).

Proof of Theorem 8.5. It follows from Lemma 8.9 and Lemma 8.10 that for any $u_{0} \in W_{0}^{1, q}(\Omega)$ every solution $u \in \operatorname{AC}\left(0, T ; L^{p}(\Omega)\right)$ of the Cauchy problem (8.2) associated with the functionals (8.19) and (8.22) is indeed a solution to Problem 8.4. Since conditions $(\mathrm{N})-(\Psi)$ of Section 8.1 are fulfilled, Theorem 8.3 thus yields the existence of a solution $u$ to the latter initial-boundary value problem. As a consequence of the energy identity (8.5), of (8.19), (8.23), (6.23), and (8.28), $u$ fulfils the energy inequality

$$
\begin{aligned}
& \frac{1}{p^{\prime}} \int_{s}^{t} \int_{\Omega} \mathcal{R}_{*}\left(u(x, r), \operatorname{div}(\beta(\nabla u(x, r)))-W^{\prime}(u(x, r))+h(x, r)\right) \mathrm{d} x \mathrm{~d} r \\
& \quad+\frac{1}{p} \int_{s}^{t} \int_{\Omega} \mathcal{R}\left(u(x, r), u_{t}(x, r)\right) \mathrm{d} x \mathrm{~d} r+\mathcal{E}_{t}(u(t)) \\
& =\mathcal{E}_{s}(u(s))+\int_{s}^{t}\left\langle h^{\prime}(r), u(r)\right\rangle \mathrm{d} r \quad \forall 0 \leq s \leq t \leq T .
\end{aligned}
$$

In particular, thanks to (8.11) we conclude that $u \in W^{1, p}\left(0, T ; L^{p}(\Omega)\right)$, while estimates (8.29), (8.32) and $\sup _{t \in[0, T]} \mathcal{E}_{t}(u(t))<+\infty$ yield (8.15). Finally, recalling (8.25) we also deduce an estimate for $-\operatorname{div}(\beta(\nabla u))+W_{c}^{\prime}(u)-h$ in $L^{p^{\prime}}\left(0, T ; L^{p^{\prime}}(\Omega)\right)$, and (8.16) ensues.

\section{References}

[1] R. A. AdAms, "Sobolev Spaces", Pure and Applied Mathematics, Academic Press, New York-London, 1975.

[2] A. Ambrosetti and G. Prodi, "A Primer of Nonlinear Analysis", Cambridge Studies in Advanced Mathematics, Cambridge University Press, Cambridge, 1993. 
[3] L. Ambrosio, Metric space valued functions of bounded variation, Ann. Scuola Norm. Sup. Pisa Cl. Sci. (4) 17 (1990), 439-478.

[4] L. Ambrosio, Minimizing movements, Rend. Accad. Naz. Sci. XL Mem. Mat. Appl. (5) 19 (1995), 191-246.

[5] L. Ambrosio, N. Gigli and G. Savaré, "Gradient Flows in Metric Spaces and in the Wasserstein Spaces of Probability Measures", Lecture notes, ETH, Birkhäuser, 2005.

[6] T. ARAI, On the existence of the solution for $\partial \phi\left(u^{\prime}(t)\right)+\partial \psi(u(t)) \ni f(t)$, J. Fac. Sci. Univ. Tokyo Sect. IA Math. 26 (1979), 75-96.

[7] M. Aso, M. Frémond and N. KenMochi, Quasi-variational evolution problems for irreversible phase change, In: "Nonlinear Partial Differential Equations and their Applications", GAKUTO Internat. Ser. Math. Appl., Gakkōtosho, Tokyo, 2004, 517-535.

[8] M. Aso, M. Frémond and N. KenMochi, Phase change problems with temperaturedependent constraints for the volume fraction velocities, Nonlinear Anal. 60 (2005), 10031023.

[9] H. Aтtоuch, "Variational Convergence for Functions and Operators", Applicable Mathematics Series, Pitman (Advanced Publishing Program) Boston MA, 1984.

[10] E. J. BALDER, A general approach to lower semicontinuity and lower closure in optimal control theory, SIAM J. Control Optim. 22 (1984), 570-598.

[11] E. J. BALDER, An Extension of Prokhorov's Theorem for Transition Probabilities with Applications to Infinite-Dimensional Lower-Closure Problems, Rend. Circ. Mat. Palermo 34 (1985), 427-447.

[12] J. M. BALL, A version of the fundamental theorem for Young measures, In: "PDEs and Continuum Models of Phase Transitions" (Nice 1988), Lecture Notes in Phys., Vol. 344, Springer, Berlin, 1989, 207-215.

[13] V. BARBU, Existence theorems for a class of two point boundary problems, J. Differential Equations 17 (1975), 236-257.

[14] C. Castaing and M. Valadier, "Convex Analysis and Measurable Multifunctions", Springer, Berlin-New York, 1977.

[15] P. Colli and A. Visintin, On a class of doubly nonlinear evolution equations, Comm. Partial Differential Equations 15 (1990), 737-756.

[16] P. COLLI, On some doubly nonlinear evolution equations in Banach spaces, Japan J. Indust. Appl. Math. 9 (1992), 181-203.

[17] E. De Giorgi, New problems on minimizing movements, In: "Boundary Value Problems for PDE and Applications", Claudio Baiocchi and Jacques Louis Lions (eds.), Masson, Paris, 1993, 81-98.

[18] E. De Giorgi, A. MARino and M. Tosques, Problems of evolution in metric spaces and maximal decreasing curve, Atti Accad. Naz. Lincei Rend. Cl. Sci. Fis. Mat. Natur. (8) 68 (1980), 180-187.

[19] G. Dal Maso, A. DeSimone and M. G. Mora, Quasistatic evolution problems for linearly elastic-perfectly plastic materials, Arch. Ration. Mech. Anal. 180 (2006), 237-291.

[20] G. Dal Maso, A. DeSimone, M. G. Mora and M. Morini, A vanishing viscosity approach to quasistatic evolution in plasticity with softening, to appear in Arch. Ration. Mech. Anal.

[21] G. DAl MAso, G. FrancFort and R. TOADER, Quasistatic growth in nonlinear elasticity, Arch. Ration. Mech. Anal. 176 (2005), 165-225.

[22] M. EfendieV and A. MielKe, On the rate-independent limit of systems with dry friction and small viscosity, J. Convex Anal. 13 (2006), 151-167.

[23] L. C. Evans and R. Gariepy, "Measure Theory and Fine Properties of Functions", Studies in Advanced Mathematics, CRC Press, Boca Raton FL, 1992.

[24] G. FRANCFORT and A. MIELKE, Existence results for a class of rate-independent material models with nonconvex elastic energies, J. Reine Angew. Math. 595 (2006), 55-91.

[25] P. Germain, "Cours de Mécanique des Milieux Continus. Tome I: Théorie Générale", Masson et Cie Éditeurs, Paris, 1973. 
[26] M. Giaquinta, "Multiple Integrals in the Calculus of Variations", Annals of Mathematics Studies, Princeton University Press, Princeton, NJ, 1983.

[27] A. MARINo, C. SACCON and M. TosQues, Curves of maximal slope and parabolic variational inequalities on nonconvex constraints, Ann. Scuola Norm. Sup. Pisa Cl. Sci. (4) 16 (1989), 281-330.

[28] A. MAINIK and A. MIELKE, Existence results for energetic models for rate-independent systems, Calc. Var. Partial Differential Equations 22 (2005), 73-99.

[29] A. Mielke, Finite elastoplasticity Lie groups and geodesics on $S L(d)$, In: "Geometry, Mechanics, and Dynamics", Springer, New York, 2002, 61-90.

[30] A. MielKE, Energetic formulation of multiplicative elasto-plasticity using dissipation distances, Contin. Mech. Thermodyn. 15 (2003), 351-382.

[31] A. MIELKE, Evolution of rate-independent inelasticity with microstructure using relaxation and Young measures, In: "IUTAM Symposium on Computational Mechanics of Solid Materials at Large Strains" (Stuttgart, 2001), Solid Mech. Appl. 108, Kluwer Acad. Publ., Dordrecht, 2003, 33-44.

[32] A. MielKe, Existence of minimizers in incremental elasto-plasticity with finite strains, SIAM J. Math. Anal. 36 (2004), 384-404.

[33] A. MiELKE, Evolution of rate-independent systems, In: "Evolutionary Equations", Vol. II, Handb. Differ. Equ., Elsevier/North-Holland, Amsterdam, 2005, 461-559.

[34] A. Mielke and M. ORTiz, A class of minimum principles for characterizing the trajectories and the relaxation of dissipative systems, to appear in ESAIM Control Optim. Calc. Var., published online: 21 December 2007, DOI: 10.1051/cocv: 2007064.

[35] A. MIELKE and R. Rossi, Existence and uniqueness results for a class of rate-independent hysteresis problems, Math. Models Methods Appl. Sci. 17 (2007), 81-123.

[36] A. Mielke, R. Rossi and G. SAVARÉ, Modeling solutions with jumps for rate-independent systems on metric spaces, in preparation.

[37] A. Mielke, R. Rossi and G. SaVAré, On the vanishing viscosity limit for the metric approach to rate-independent problems, in preparation.

[38] A. MielKe and T. ROUBIČEK, A rate-independent model for inelastic behavior of shapememory alloys, Multiscale Model. Simul. 1 (2003), 571-597.

[39] A. MiELKE and T. ROUBIČEK, Rate-independent damage processes in nonlinear elasticity, Math. Models Methods Appl. Sci. 16 (2006), 177-209.

[40] A. MiELKE and F. THEIL, A mathematical model for rate-independent phase transformations with hysteresis, In: Proceedings of the Workshop on "Models of Continuum Mechanics in Analysis and Engineering", H.-D. Alber, R. Balean and R. Farwig (eds.), ShakerVerlag, Aachen, 1999, 117-129.

[41] A. Mielke, F. Theil and V. Levitas, A variational formulation of rate-independent phase transformations using an extremum principle, Arch. Ration. Mech. Anal. 162 (2002), $137-177$.

[42] A. MielKe and F. THEIL, On rate-independent hysteresis models, NoDEA Nonlinear Differential Equations Appl. 11 (2004), 151-189.

[43] J. J. Moreau, Sur l'évolution d'un système élasto-visto-plastique, C. R. Acad. Sci. Paris Sér. A-B 273 (1971), A118-A121.

[44] L. Nirenberg, On elliptic partial differential equations, Ann. Scuola Norm. Sup. Pisa Cl. Sci. (3) 13 (1959), 115-162.

[45] R. Rossi and G. SAVARÉ, Gradient flows of non convex functionals in Hilbert spaces and applications, ESAIM Control Optim. Calc. Var. 12 (2006), 564-614.

[46] G. Schimperna, A. Segatti and U. Stefanelli, Well-posedness and long-time behavior for a class of doubly nonlinear equations, Discrete Contin. Dyn. Syst. 18 (2007), $15-38$.

[47] A. SEGATTI, Global attractor for a class of doubly nonlinear abstract evolution equations, Discrete Contin. Dyn. Syst. 14 (2006), 801-820.

[48] T. SEnBA, On some nonlinear evolution equations, Funkcial. Ekva. 29 (1986), 243-257. 
[49] U. STEFANELLI, The Brézis-Ekeland principle for doubly nonlinear equations, to appear in SIAM J. Control Optim.

[50] M. VAladieR, A course on Young measures, Workshop on Measure Theory and Real Analysis (Italian) (Grado, 1993), Rend. Istit. Mat. Univ. Trieste 26 (1994), suppl. (1995), 349394.

[51] A. Visintin, A new approach to evolution, C. R. Acad. Sci. Paris Sér. I Math. 332 (2001), 233-238.

Dipartimento di Matematica

Università di Brescia

via Valotti, 9

I-25133 Brescia, Italy

riccarda.rossi @ ing.unibs.it

Weierstraß-Institut

Mohrenstraße 39

D-10117 Berlin, Germany

Institut für Mathematik

Humboldt-Universität zu Berlin

Rudower Chaussee, 25

D-12489 Berlin (Adlershof), Germany

mielke@wias-berlin.de

Dipartimento di Matematica

"F. Casorati"

Università di Pavia

via Ferrata, 1

I-27100 Pavia, Italy

giuseppe.savare @ unipv.it 